\title{
Efficient and Precise Genome Editing in Shewanella with Recombineering and CRISPR/Cas9-mediated Counter-selection
}

\author{
A DISSERTATION \\ SUBMITTED TO THE FACULTY OF \\ THE UNIVERSITY OF MINNESOTA \\ BY
}

Anna Domenech Corts

IN PARTIAL FULFILLMENT OF THE REQUIREMENTS

FOR THE DEGREE OF

DOCTOR OF PHILOSOPHY

Advisor: Jeffrey A. Gralnick

June 2019 
(C) Anna Domenech Corts 2019 


\section{ACKNOWLEDGEMENTS}

The work presented in this dissertation would not have been possible without the support of many people. First, I would like to thank my advisor Jeffrey Gralnick, for his continuous support and enthusiasm throughout my time in the UMN. I deeply appreciate your trust in me!

I would like to thank my committee members, Katie Fixen, Kyle Costa, Dan Voytas and Jeff Gralnick for their support. I specially thank Katie Fixen for the several times you took me out for coffee to chat. I really appreciate listening to your story, your advice and I am thankful for the encouragement you have given me.

Thanks to the department of Plant and Microbial Biology for giving me the opportunity to join such a great intellectual and friendly environment. I specially thank Sara Eliason, for her honest support and care for the students in the program.

I would like to thank my mentor Jill Tietjen. Without you, I wouldn't have made it. Thank you for your love and for always believing in me and lifting me up!

Thanks to Lynn Thomason for all your constant advice and support throughout my thesis, and, especially, for being my first reader on everything! I am so glad you came to Minneapolis and I met you!

Also, thanks to all my labmates in the Gralnick and Bond labs for creating such a welcoming environment. Special thanks to Rebecca Calvo for all your support, you have been like a sister for me. Thanks to Chi Ho Chan, for always helping me with my research and Eric Kees, for always getting my oligos! I would like to also thank Ruth Lee and Maya Burroughs for listening again and again while I practiced my talks and for your feedback! 


\begin{abstract}
Shewanella are invaluable hosts for the discovery and engineering of pathways important for bioremediation of toxic and radioactive metals, to create microbial fuel cells and for understanding extracellular electron transfer. However, studies on this species have suffered from a lack of effective genetic tools for precise and high throughput genome manipulation. Previously, the only reliable method used for introducing DNA into Shewanella spp. at high efficiency was bacterial conjugation, enabling transposon mutagenesis and targeted knockouts using suicide vectors for gene disruptions.

In this dissertation, I describe development of simple and efficient genome editing tools for precise and site-directed mutagenesis of Shewanella. First, In Chapter I, I review recent advances in synthetic biology that accelerate the study and engineering of bacterial phenotypes. In chapter II, I show the development of a robust and simple electroporation method in S. oneidensis that allows an efficiency of up to $\sim 10^{8}$ transformants/ $\mu \mathrm{g}$ DNA and which is adaptable to other strains. Using this method for DNA transfer, in chapter III, I characterize a new phage recombinase, W3 Beta from Shewanella sp. W3-18-1 and show its utility for in vivo genome engineering (recombineering) using linear single-stranded DNA oligonucleotides. In my experiments the W3 Beta recombinase gives an efficiency of $\sim 5 \%$ recombinants among total viable cells. In addition, I show the functionality of this new system in S. amazonensis, a strain with few genetic studies but of interest given its higher temperature range for growth and wide range of carbon sources utilized. In chapter IV, I demonstrate use of the CRISPR/Cas9 system as a counter-selection to isolate recombinants. When coupled to recombineering, this counter-selection results in an extremely high efficiency of $>90 \%$ among total surviving cells, regardless of the gene or strain modified. This efficiency allows isolation of several different types of mutations made with recombineering, and even allows identification of rare recombinants that form independently of W3 Beta expression. This is the first effective and simple strategy for recombination with markerless mutations in Shewanella. With synthesized single-stranded DNA as substrates for homologous recombination and CRISPR/Cas9 as a counter-selection, this new system provides a rapid, scalable, versatile and scarless tool that will accelerate progress in Shewanella genomic engineering. Finally, I conclude in Chapter $\mathrm{V}$ with an overview of the challenges and future directions of the technologies demonstrated here, discussing possible advancements that could further enhance the study of Shewanella.
\end{abstract}




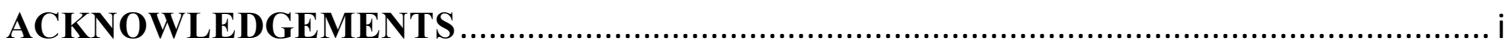

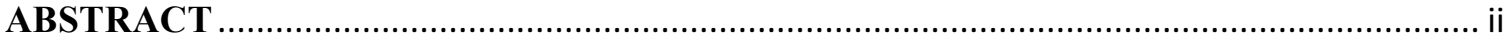

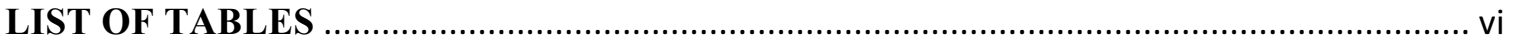

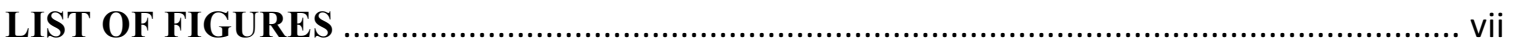

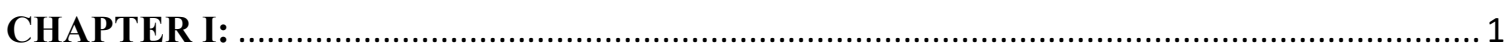

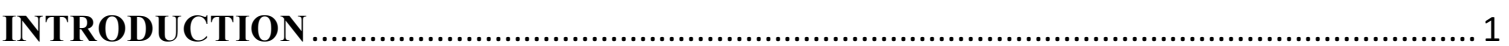

1.1. The respiratory versatility of Shewanella .............................................................. 1

1.2. CRISPR-based editing technologies in bacteria ...................................................... 2

1.3. Genome Editing Technologies for Shewanella ....................................................... 4

1.4. CRISPR EnAbled Trackable genome Engineering (CREATE) ................................ 6

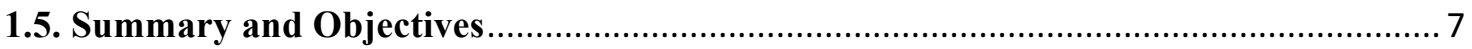

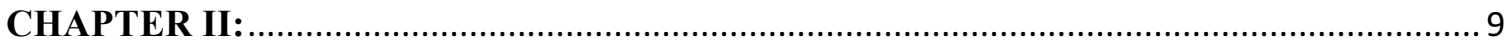

DEVELOPMENT AND OPTIMIZATION OF AN EFFICIENT

ELECTROTRANSFORMATION METHOD IN SHEWANELLA ….............................. 9

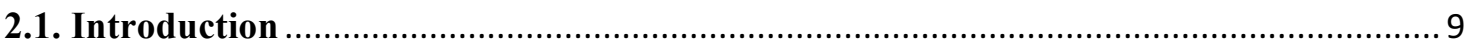

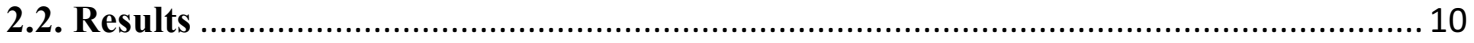

2.2.1. Room temperature enhances electrotransformation efficiency in S. oneidensis .. 10

2.2.2. Development of an efficient electroporation method in $\boldsymbol{S}$. oneidensis .................. 12

2.2.3. Effect of plasmid DNA methylation patterns on electroporation efficiency …...... 15

2.2.4. Application of electroporation to other Shewanella strains ................................. 16

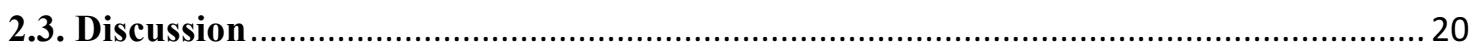

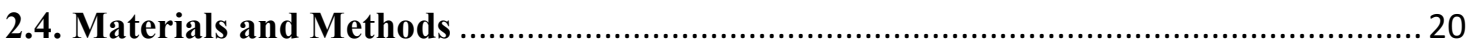

2.4.1. Bacterial Strains, plasmids and culture conditions ........................................... 20

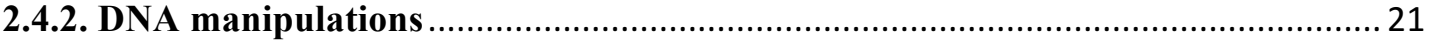

2.4.3. Preparation of competent cells and electrotransformation................................... 22

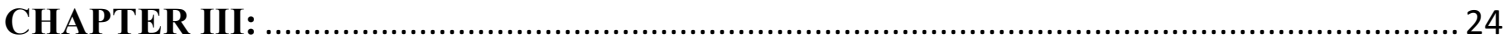

A NEW RECOMBINEERING SYSTEM FOR PRECISE GENOME-EDITING IN SHEWANELLA USING SINGLE-STRANDED OLIGONUCLEOTIDES......................... 24

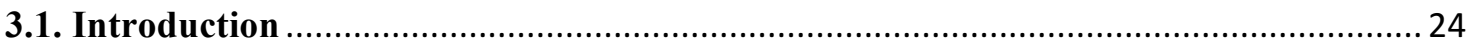

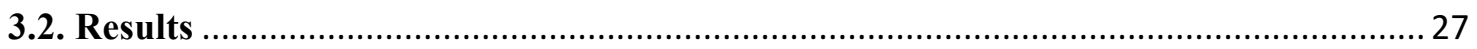

3.2.1. W3 Beta from Shewanella sp. W3-18-1 is homologous to $\lambda$-Red Beta .................. 27

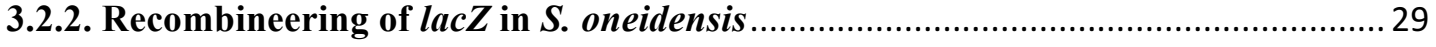




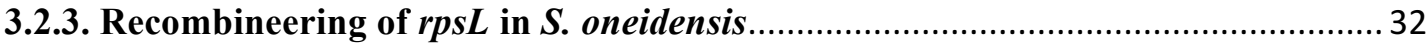

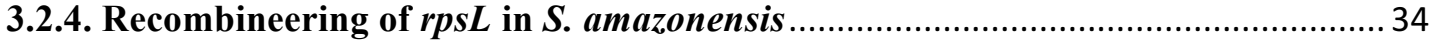

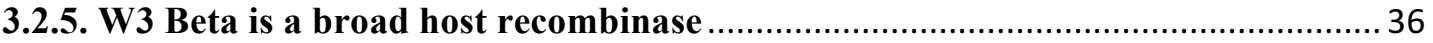

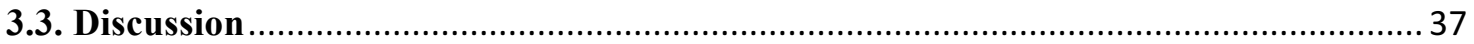

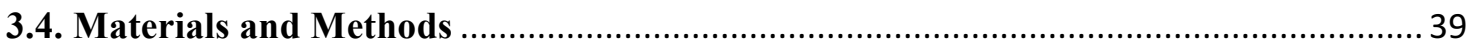

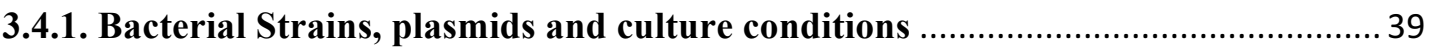

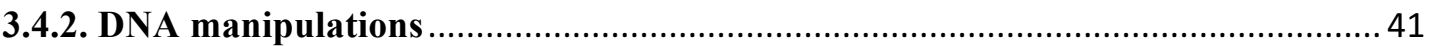

3.4.3. ssDNA oligo recombineering in $S$. oneidensis and $S$. amazonensis ..................... 42

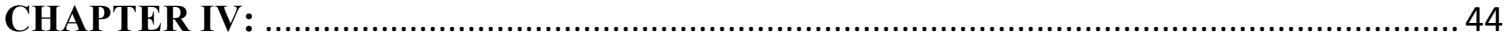

EFFICIENT AND PRECISE GENOME EDITING IN SHEWANELLA THROUGH

RECOMBINEERING AND CRISPR/CAS9-MEDIATED COUNTERSELECTION ......... 44

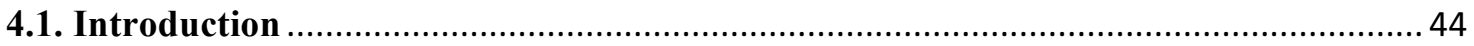

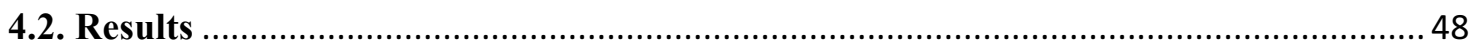

4.2.1. Lethality of DSB Induced by Active Cas9 in Shewanella ................................... 48

4.2.2. Recombinase-independents ssDNA genome editing coupled to CRISPR/Cas9

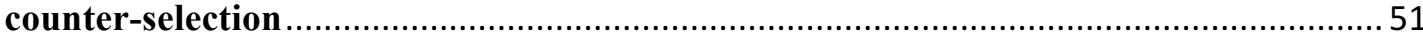

4.2.3. SsDNA recombineering coupled to CRISPR/Cas9 counter-selection gives high

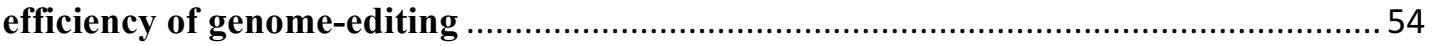

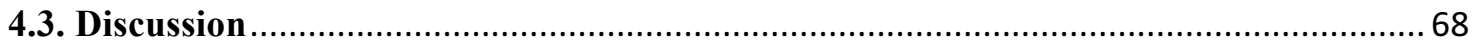

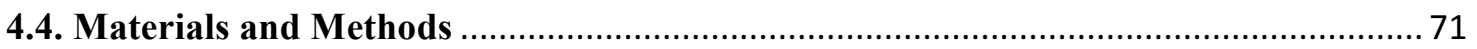

4.4.1. Strains, plasmids, oligonucleotides and growth conditions................................. 71

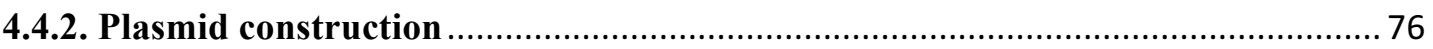

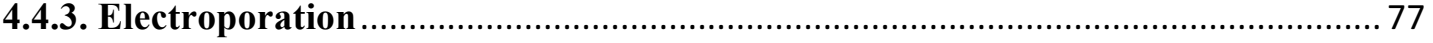

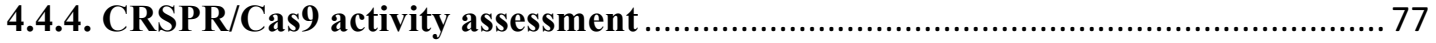

4.4.5. ssDNA genome-editing combined with CRISPR/Cas9 counter-selection ............. 78

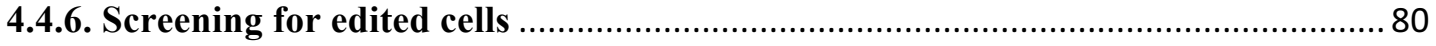

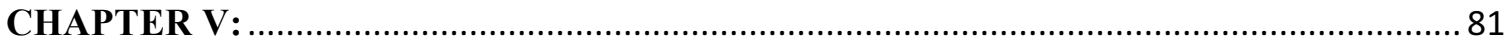

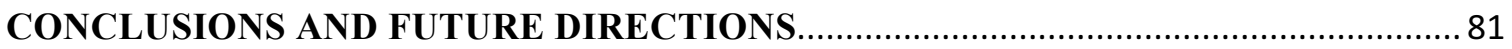

5.1. Application of electro-transformation in different Shewanella strains...................... 81

5.2. Use of recombineering for different types of DNA changes........................................ 82

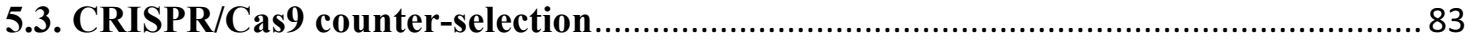

5.4. Large-scale genome engineering using recombineering coupled to CRISPR/Cas9 .... 83

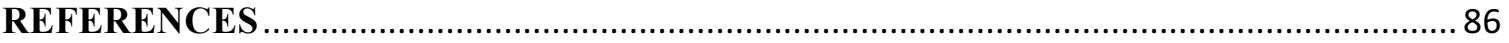


Note: Some of the contents of this work have been adapted from Anna D. Corts et al., Nature Sci. Rep., "A new recombineering system for precise genome-editing in Shewanella oneidensis strain MR-1 using single-stranded oligonucleotides" (2019), and paper submitted, Anna D. Corts et. al., ACS SynBio, "Efficient and Precise Genome Editing in Shewanella with Recombineering and CRISPR/Cas9-mediated Counter-selection” 


\section{LIST OF TABLES}

\section{Tables for Chapter II}

Table 2. 1. Gralnick Lab high efficiency electroporation protocol for plasmid transformation in $S$.

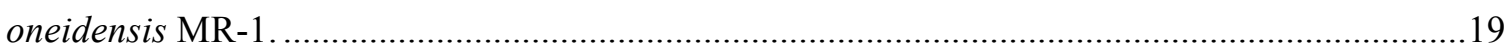

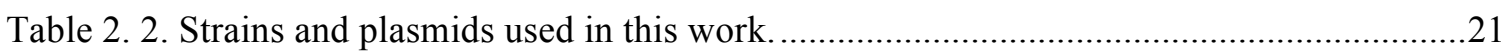

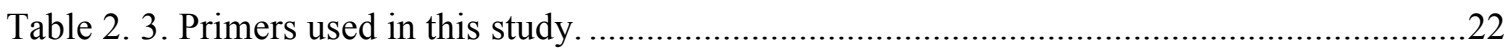

\section{Tables for Chapter III}

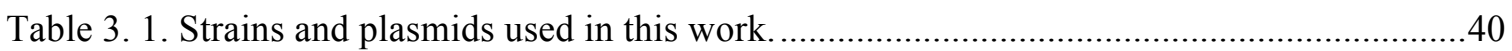

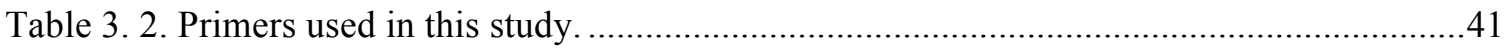

Table 3. 3. Oligonucleotides used for chromosomal mutations...................................................43

\section{Tables for Chapter IV}

Table 4. 1. Genome editing of different genes in S. oneidensis and S. amazonensis....................59

Table 4. 2. Comparison of different genome editing techniques in Shewanella ...........................70

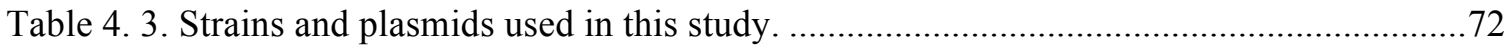

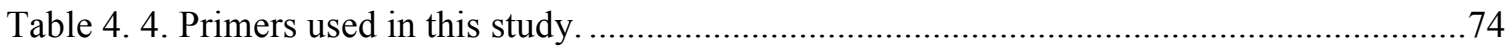

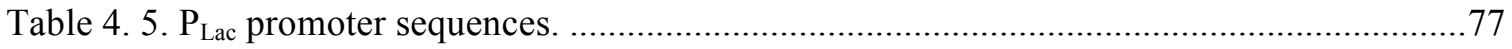

Table 4. 6. Oligonucleotides used for homologous recombination. ............................................79

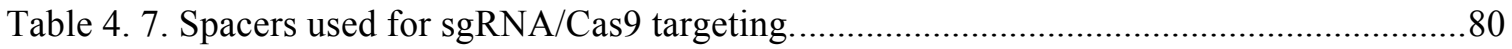

Table 4. 8. gBLOCKS containing the synthetic sgRNA sequences. .......................................... 80 


\section{LIST OF FIGURES}

\section{Figures for Chapter I}

Figure 1. 1. Outline of the Mtr pathway in S. oneidensis MR-1.............................................. 2

Figure 1. 2. Recombineering coupled to CRISPR/Cas9 counter-selection. .................................... 4

Figure 1.3. Timeline of advances contributing to genome-engineering technologies in

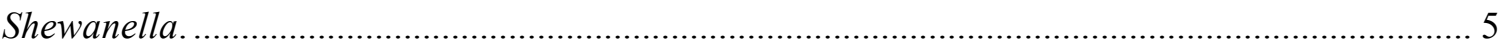

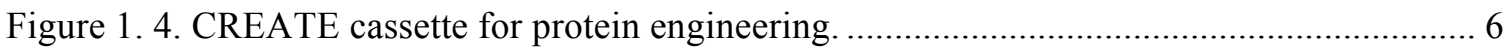

Figure 1. 5. Overview of the CREATE workflow.................................................................. 7

\section{Figures for Chapter II}

Figure 2. 1. Electrotransformation efficiency and cell viability at room temperature (RT) and ice temperature (IT).

Figure 2. 2. Comparison of electrotransformation at room temperature (RT) and ice temperature

(IT)

Figure 2. 3. Effects of various parameters on $S$. oneidensis transformation

Figure 2. 4. Effect of time frozen in $1 \mathrm{M}$ sorbitol at $-80{ }^{\circ} \mathrm{C}$ on $S$. oneidensis

electrotransformation. 15

Figure 2. 5. Effect of plasmid DNA methylation patterns on electroporation efficiency 16

Figure 2. 6. Electroporation efficiencies in different Shewanella strains compared to E. coli......18

\section{Figures for Chapter III}

Figure 3. 1. Representation of the stepwise approach used for recombineering in S. oneidensis. .26

Figure 3. 2. Effect of recombinase expression on S. oneidensis growth. .28

Figure 3. 3. Recombinase protein alignments.. .29

Figure 3. 4. Disruption of chromosomal lacZ in S. oneidensis. .30

Figure 3. 5. Recombineering of lacZ in S. oneidensis using W3 Beta recombinase. .31

Figure 3. 6. Mutagenesis of chromosomal rpsL in S. oneidensis. .32

Figure 3. 7. Recombineering of $r p s L$ in $S$. oneidensis using W3 Beta recombinase. .33

Figure 3. 8. S. oneidensis rpsLK43R recombinants verification. .34

Figure 3. 9. Mutagenesis of chromosomal rpsL in S. amazonensis. 35 
Figure 3. 10. Recombineering in S. amazonensis SB2B.....

Figure 3. 11. ssDNA recombineering in E. coli MG1655 by preparing electrocompetent cells at different temperatures. .....

\section{Figures for Chapter IV}

Figure 4. 1. Overview of ssDNA recombineering combined with CRISPR/Cas9 as a counterselection. .47

Figure 4. 2. Effect of Cas9 expression on S. oneidensis growth. .49

Figure 4. 3. Lethality of DSBs induced by active Cas9 in Shewanella. .50

Figure 4. 4. Overview of CRISPR/Cas9 counter-selection coupled with recombinase-independent ssDNA genome-editing.

Figure 4. 5. Recombinase-independent ssDNA genome-editing coupled to CRISPR/Cas9 counterselection.

Figure 4. 6. Verification of lacZ edits in S. oneidensis using CRISPR/Cas9 counter-selection coupled with recombinase-independent ssDNA genome-editing. .53

Figure 4. 7. Plasmid maps of the two-plasmid based system.

Figure 4. 8. Representation of lagging strand-targeting oligos in S. oneidensis MR-1 and $S$. amazonensis $\mathrm{SB} 2 \mathrm{~B}$ genomes.

Figure 4. 9. ssDNA recombineering combined with CRISPR/Cas9 as a counter-selection in $S$. oneidensis.

Figure 4. 10. Schematics of the mutations created by ssDNA recombineering coupled to CRISPR/Cas9 counter-selection.

Figure 4. 11. Verification of $10 \mathrm{bp}$ lac Z, $m$ trA and exeM mutants by recombineering combined with CRISPR/Cas9 counter-selection.

Figure 4. 12. Verification of sama_1213 mutants by recombineering combined with

CRISPR/Cas9 counter-selection using different size oligos.

Figure 4. 13. Verification of $m t r A$ deletion mutants by recombineering combined with

CRISPR/Cas9 counter-selection using different size oligos.

Figure 4. 14. Verification of exeM point mutation by recombineering combined with

CRISPR/Cas9 counter-selection using different size oligos . .65

Figure 4. 15. Iron reduction test of $m \operatorname{tr} A$ deletion mutants.....................................................66

Figure 4. 16. System optimization. .67 


\section{CHAPTER I: \\ INTRODUCTION}

\subsection{The respiratory versatility of Shewanella}

Members of the genus Shewanella form a group of facultative anaerobic gram negative bacteria widely distributed in marine and freshwater environments (Venkateswaran et al. 1999; Hau \& Gralnick 2007). The hallmark of many Shewanella is the ability to utilize a remarkably diverse array of final electron acceptors in the absence of oxygen, which allows them to survive in extreme and varied environments. As a genus, these organisms have the most diverse respiratory abilities described to date (Hau \& Gralnick 2007; Venkateswaran et al. 1999). The approximately 20 organic and inorganic compounds that can be respired by Shewanella include a number of toxic elements and insoluble metals (Satomi 2014; Fredrickson et al. 2008). As a result, they show great potential for remediation of various environmental pollutants (Tiedje 2002) and in microbial fuel cells, where their metabolism is harnessed to make electricity from waste water treatment (Rabaey \& Verstraete 2005; Lovley 2012).

S. oneidensis MR-1, formerly Shewanella putrefaciens MR-1, is the best characterized strain of Shewanella (Heidelberg et al. 2002). This bacterium was isolated in 1987 from sediment from Oneida Lake (Myers \& Nealson 1988), New York, and it is found in numerous sedimentary environments. As in other species, S. oneidensis uses oxygen as the terminal electron acceptor during aerobic respiration; however, under anaerobic conditions, it undertakes respiration by reducing alternative terminal electron acceptors. A partial list includes oxidized metals (including $\mathrm{Mn}(\mathrm{III})$ and (IV), Fe(III), $\mathrm{Cr}(\mathrm{VI}), \mathrm{U}(\mathrm{VI})$ ), fumarate, nitrate, trimethylamine $N$-oxide, dimethyl sulfoxide, sulfite, thiosulfate, elemental sulfur and more. A complete list can be found elsewhere (Beblawy et al. 2018). Such plasticity in alternative electron acceptors for anaerobic respiration has not been observed in any other organism so far.

The anaerobic respiratory versatility of $\mathrm{S}$. oneidensis is a consequence of its multicomponent branched electron transport system (Breuer et al. 2015). Of particular interest is the metal respiratory (Mtr) pathway, which consists of at least five multiheme $c$-type cytochromes (CymA, FccA, STC, MtrA and MtrC) and a porin-like outer membrane protein MtrB (Figure 1.1). Cytochrome CymA oxidizes quinol in the cytoplasmic membrane and transfers electrons to periplasmic cytochromes FccA and STC, which distribute the electrons to MtrA (Brutinel \& Gralnick 2012b). A trans-outer membrane protein complex formed by MtrA, MtrB and MtrC 
transfers the electrons from the periplasm to the extracellular space, where MtrC functions as a terminal reductase that interacts with the metal substrates (Beblawy et al. 2018). Unlike other pathways, the Mtr pathway is known to be promiscuous; it can reduce 9,10-anthraquinone-2,6disulfonic acid (AQDS) and carbon electrodes, as well as soluble substrates that form solids upon reduction, such as chelated cobalt, vanadium(V), uranium(VI), technetium(VII) (Beblawy et al. 2018; Shi et al. 2012; Shi et al. 2016). Development of efficient genome-editing tools allowing sitedirected mutagenesis could help clarify the molecular mechanisms involved in the Mtr pathway and promote a better understanding of this promiscuity. Some engineering approaches have been applied for large-scale manipulation of Shewanella, although these were based on random mutagenesis (Yang et al. 2014; Qiu et al. 2004; Jones et al. 2010), which is useful for gene disruption but does not allow the manipulation of essential genes. To better understand the current extracellular electron transfer model and to fully exploit the metabolic potential of this family, easy and efficient methods for rational genome engineering are needed.

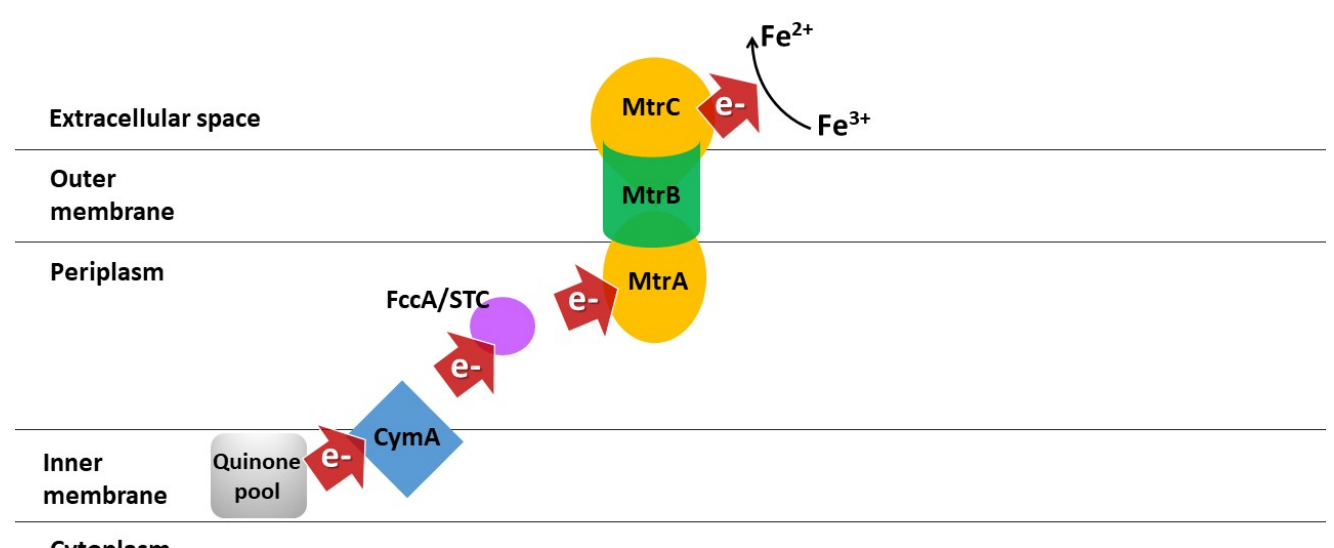

Cytoplasm

Figure 1. 1. Outline of the Mtr pathway in S. oneidensis MR-1.

Multiheme $c$-type cytochromes CymA, FccA/STC, MtrA, MtrC and the porin-like outer membrane protein MtrB form an electron conduit to transfer the electrons from carbon source oxidation to the outside of the cell. MtrC can reduce insoluble metal substrates in the extracellular space.

\subsection{CRISPR-based editing technologies in bacteria}

Recently, the CRISPR (clustered regularly interspaced short palindromic repeats)- Cas (CRISPR associated) systems have revolutionized genome engineering in a broad range of organisms, from bacteria (Wendt et al. 2016; Wolf et al. 2016; Huang et al. 2015; Tong et al. 2015; 
Altenbuchner 2016; Cobb et al. 2014; Mougiakos et al. 2017), yeast (Weninger et al. 2016; Ng \& Dean 2017) to mammalian cells (Richardson et al. 2016; Chu et al. 2015) and plants (Liang et al. 2016; Kuei \& Puchta 2019). Because of its inherent simplicity, the most exploited CRISPR/Cas system is the Class 2 type IIa CRISPR/Cas from S. pyogenes, SpCas9, (Hsu et al. 2015) as further explained in chapter IV. The utilization of CRISPR/Cas9 for genome engineering purposes requires two components; the nuclease Cas9 and a targeting guide RNA, gRNA (Selle \& Barrangou 2015). Targeting any site requires only the insertion of a short sequence (20bp) upstream of the gRNA scaffold, which matches the target gene sequence, denoted as protospacer, that one desires to modify. When co-expressed, the Cas9/gRNA complex can target any DNA sequence, given that a short (3nt) motif, the protospacer adjacent motif (PAM) is present downstream of the protospacer sequence. The PAM for SpCas9 is 5'-NGG-3' (Shah et al. 2013). Annealing of the gRNA spacer to the target site in the genome leads to a double stranded break (DSB) produced by the Cas9 nuclease (Selle \& Barrangou 2015).

In eukaryotic cells, the DSB introduced by Cas9 can be repaired by the nonhomologous DNA end joining (NHEJ) recombination system, resulting in a mutation at the target site (Komor et al. 2017). However, NHEJ either does not work efficiently or is not present in most bacteria (Selle \& Barrangou 2015). This may be seen as a disadvantage to using CRISPR-Cas9 technology for the inactivation of genes by small deletions or insertions (indels). On the other hand, for precise gene editing, DSBs may be repaired by homologous recombination using an engineered homologous template, a process that is more efficient in prokaryotes than in eukaryotes. Linear templates or plasmid-borne templates can be used for homologous recombination (HR) (Selle \& Barrangou 2015).

In prokaryotes, an attractive application has been the combination of in vivo recombinationmediated genome engineering (recombineering) with the use of CRISPR/Cas9 as a counterselection tool (Figure 1.2), which has been shown to result in highly efficient genome engineering (Yan et al. 2017; Jiang et al. 2013; Ronda et al. 2016; Paix et al. 2016). Recombineering harnesses phage recombination proteins for HR of linear DNA donor templates with the bacterial chromosome (Sharan et al. 2009). Two recombineering systems have been well characterized for genome engineering in E. coli; the $\lambda$-Red from phage $\lambda$ and the RacET from the E. coli Rac prophage, each which contain an exonuclease associated with a recombinase (Pines et al. 2015; Boyle et al. 2013) that promote HR with the chromosome. Unlike other homologous recombination techniques, recombineering allows to efficiently recombine DNA with homologous regions as short as $30-50 \mathrm{bp}$, and many mutations can be created, including insertions, deletions or substitutions. Linear donor templates in the form of single-stranded DNA (ssDNA) oligonucleotides (oligos) or 
double-stranded DNA (dsDNA) PCR products can be used (Ellis et al. 2001; Yu et al. 2000). When dsDNA is introduced, both the exonuclease ( $\lambda$-Exo or RecE from the Rac prophage) and its associated recombinase ( $\lambda$-Beta or RecT) are required (Datta et al. 2008), but if ssDNA oligos are used, only the recombinase is needed (Ellis et al. 2001). Coupling recombineering to CRISPR/Cas9 in bacteria results in a powerful hybrid technology as CRISPR/Cas9 here acts as a negative selection; no selectable markers are necessary because non-edited cells will die after the DSB (Mougiakos et al. 2016).

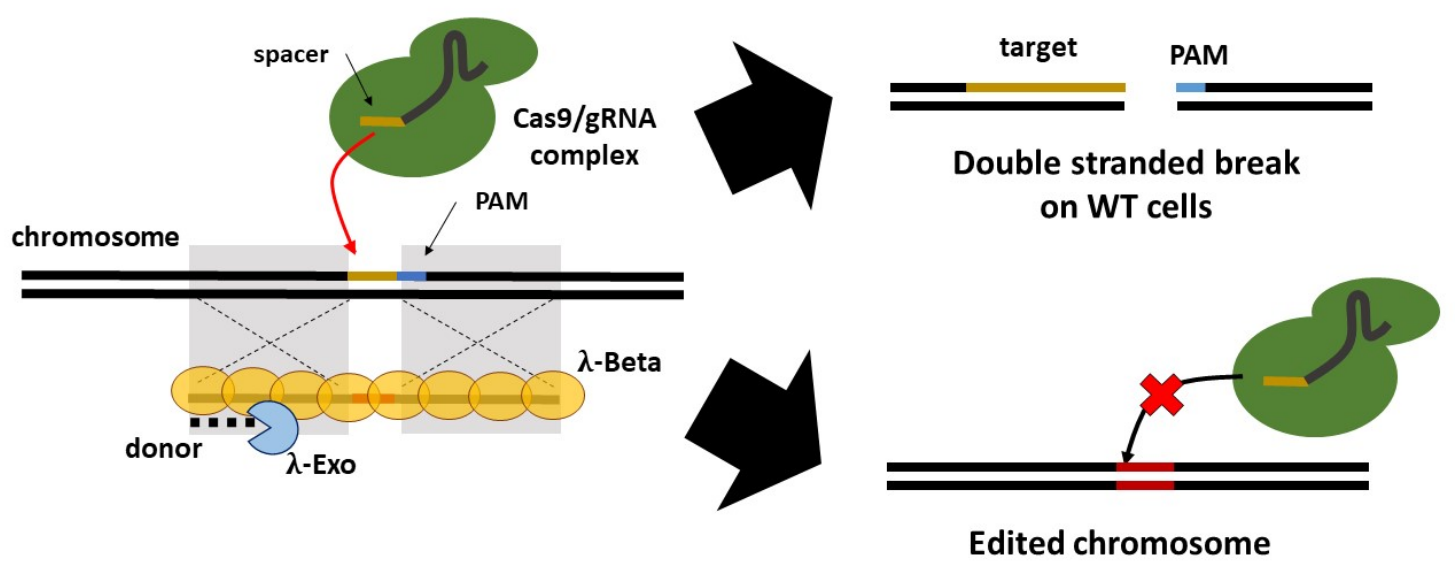

Figure 1. 2. Recombineering coupled to CRISPR/Cas9 counter-selection.

The exonuclease $\lambda$-Exo first processes the dsDNA donor template leaving ssDNA, which $\lambda$-Beta recombinase anneals and recombines at the target gene (Sharan et al. 2009). CRISPR/Cas9 is then induced to eliminate the remaining unmodified cells by creating a DSB in the chromosome, which host nucleases further degrade, leading to destruction of unmodified parental chromosomes. A silent mutation is usually introduced in the PAM site, in addition to the desired mutations, to abrogate Cas9 cleavage of the mutated cells and thus conferring CRISPR immunity (Garst et al. 2016).

\subsection{Genome Editing Technologies for Shewanella}

Previous genome editing tools for Shewanella include random chemical (Saffarini et al. 1994), UV (Qiu et al. 2004) or transposon mutagenesis (Deutschbauer et al. 2011; Brutinel \& Gralnick 2012a) and targeted deletions (Beliaev \& Saffarini 1998) using the traditional suicideplasmid technique (Figure 1.3). Although these tools have been useful for elucidation of important 
genotypic traits of this bacterium, for genome-scale engineering these are neither sufficient nor efficient, as these techniques are usually applied to create knock-out mutants.

In this dissertation, I describe characterization of a new bacteriophage recombinase protein, W3 Beta from Shewanella sp. W3-18-1 (NCBI accession WP_011788511, locus sputw3181_1153, annotated herein as $s w_{-}$1153) and demonstration of its utility for in vivo genome engineering (recombineering) in S. oneidensis and S. amazonensis, using single stranded DNA (ssDNA) oligos as donor templates for HR. Similar to $\lambda$-Beta and RecT, W3 Beta allows for the introduction of nearly any desired mutation, and requires only 40 nucleotides (nt) of flanking homology to the target gene (Corts et al. 2019), as opposed to traditional RecA-mediated double-crossover recombination with non-replicative plasmids, which requires long homology regions of $\sim 1 \mathrm{~kb}$ (Coursolle \& Gralnick 2010).

In contrast to random mutagenesis, recombineering allows modification of the genome in a precise and targeted way and, as I show in chapter III, an efficiency up to $\sim 5 \%$ recombinants among total viable cells can be obtained in $S$. oneidensis. The establishment of ssDNA recombineering has been a major advance for Shewanella genome-engineering. However, when the introduced mutation(s) do not result in a clear phenotypic change, a PCR-based screen is used to identify the recombinants, which can be cumbersome. Thus, in chapter IV, I develop a system that couples recombineering to the CRISPR/Cas9-mediated counter-selection. The addition of CRISPR/Cas9 in the system now allows to efficiently isolate the recombinant cells up to $>90 \%$ among total cells.

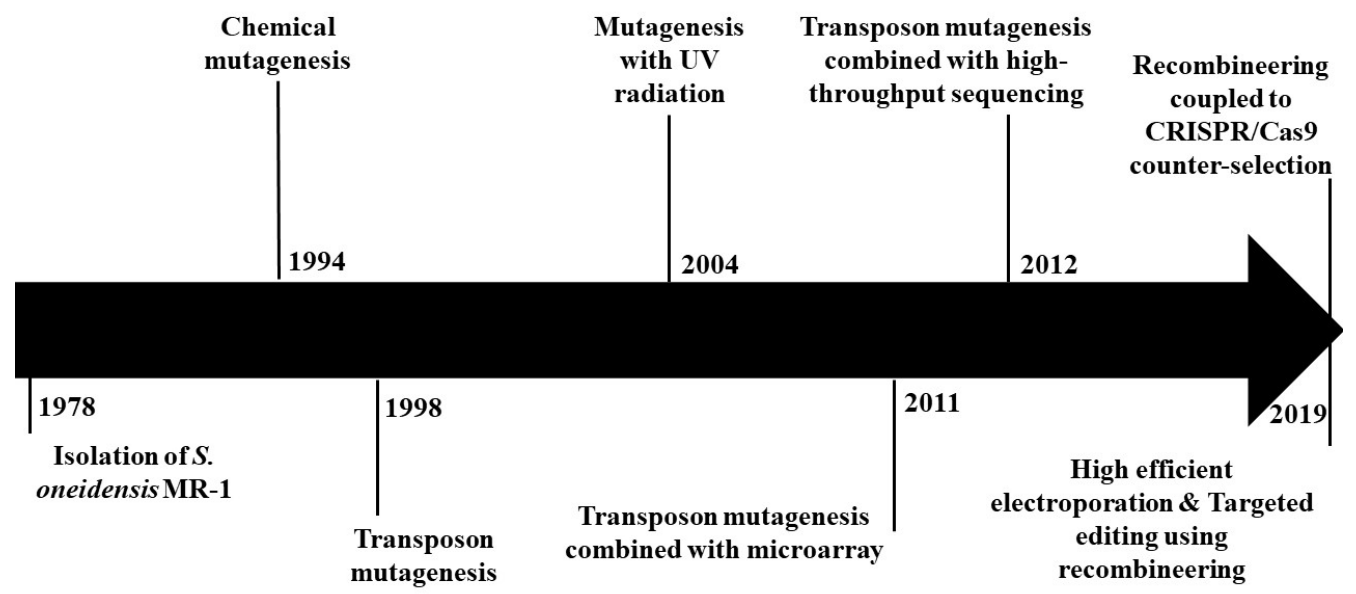

Figure 1. 3. Timeline of advances contributing to genome-engineering technologies in Shewanella.Refer to the text for details and references. 


\subsection{CRISPR EnAbled Trackable genome Engineering (CREATE)}

CREATE is a recently developed high-throughput strategy for generating thousands of precise mutant variants in E. coli (Figure 1.5) (Garst et al. 2016; Liang et al. 2017; Liu et al. 2018). This technology couples recombineering by the $\lambda$ Red system with CRISPR/Cas9 as a counterselection. A unique characteristic of CREATE is the use of a plasmid-born short donor template for homologous recombination using $\lambda$-Red. This template is located upstream of the sgRNA sequence in the sgRNA expressing plasmid, as shown in Fig. 1.4, which together with the sgRNA is called the CREATE cassette. This cassette is then used for mapping and tracking the mutations generated into the chromosome (Figure 1.5), as the plasmid carrying the CREATE cassette stays in the cell and, thus, it serves as a transacting barcode for the genomic edit, allowing parallel genotype-phenotype assessment of designs that are enriched in the population.

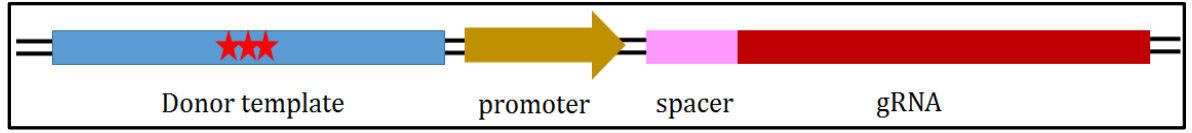

Figure 1. 4. CREATE cassette for protein engineering.

A short donor template with flanking homology arms to the targeting gene is synthesized de novo together with promoter expressing the gRNA and the gRNA complete sequence.

The CREATE strategy is convenient as thousands of CREATE cassettes can be designed in parallel by using a custom CREATE software and synthesized on a microarray platform as large pools $\left(10^{4}-10^{6}\right.$ library members $)$ at a cost of approximately $<\$ 0.001 / \mathrm{bp}$. From the microarray, the library of cassettes is amplified and cloned into a plasmid in multiplex. After introduction of the plasmid library into cells harboring Cas 9 and $\lambda$-Red, the mutations are transferred to the desired target in the genome in just a single step (Fig. 1.5). The great advantage of using this CREATE plasmid design is that it ensures that the sequences required to guide cleavage and homologous recombination are covalently coupled during the synthesis and cloning, and thus delivered simultaneously to the same cell for genome editing. 


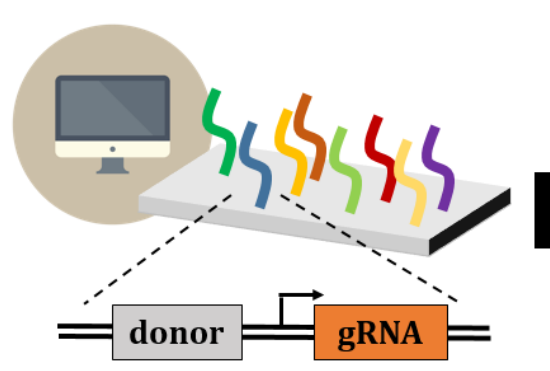

CREATE cassette

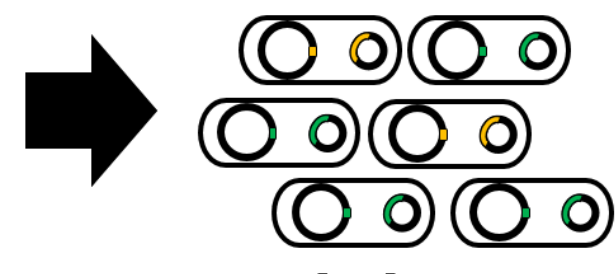

Enriched mutants
CREATE library
0.00 .00 .0
0.00 .0
0.0000 .0

Multiplexed engineering
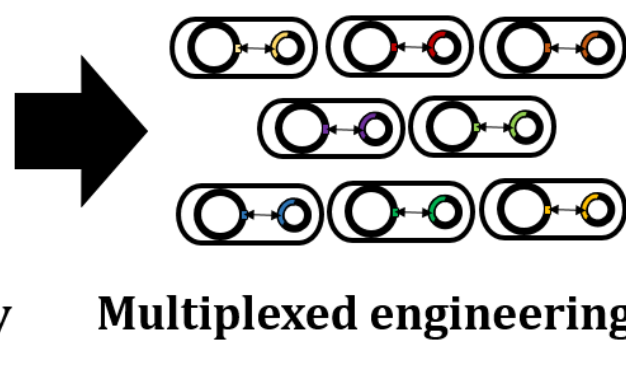

\section{Plasmid sequencing \& mapping}

Figure 1. 5. Overview of the CREATE workflow.

Library design is automated in a software package (http://www.thebioverse.org) and synthesized in parallel using microarray-based oligo technologies. The pool of DNA can be cloned in multiplex and transformed in cells harboring Cas9 and the $\lambda$-Red machinery. High-throughput sequencing of plasmid cassettes in the library before and after selective pressure can be leveraged to calculate plasmid enrichment and infer the enrichment of the corresponding genomic edit, allowing parallel genotype-phenotype mapping of edits that confer an advantage under selective pressure.

CREATE enabled the concurrent evaluation of $>50,000$ genome-wide mutations with parallel mapping in a single experiment and led to the identification of previously unreported mutations that are rarely found by adaptive laboratory evolution (ALE) or error-prone approaches (Garst et al. 2016). Additionally, the CREATE tool was employed to introduce multiple RBS variations for each of the genes in a chromosomal integrated isopropanol production pathway in $E$ .coli, leading to the time-efficient construction and testing of $\sim 1,000$ strains (Liang et al. 2017).

\subsection{Summary and Objectives}

In summary, the work described in this dissertation was designed to address the abovementioned limitations on our ability to precisely manipulate Shewanella, by developing and demonstrating technologies that facilitate genetic engineering of this organism. In order to achieve this goal, I leveraged recent advances in synthetic biology to develop novel technologies that improve (1) the ability to accurately perturb the chromosomal DNA and (2) the means to rapidly construct and isolate mutants in Shewanella. 
In Chapter II, I first describe development of a method for electro-transformation of in Shewanella spp. Until recently, the only means to transfer DNA in this bacterium was conjugation. However, here I show that electroporation is also possible in Shewanella and at very high efficiency. Several parameters were tested in order to optimize the efficiency of transformation: temperature, optical density and cell growth, amount of DNA and cells, wash buffer, and others. The advantage of electro-transformation is that not only circular DNA can be transferred, but also linear DNA in the form of dsDNA or ssDNA for genome engineering, which allows integration of exogenous DNA by recombineering.

In Chapter III, I describe discovery of a new phage protein, W3 Beta, and demonstrate its utility for high efficiency of recombineering in S. oneidensis. I provide examples for precise editing of chromosomal lacZ and rpsL and provide evidence that W3 Beta may be broader host than $\lambda$-Red Beta and RecT. Next, I demonstrate the application of recombineering in S. amazonensis. The results described in Chapters II and III were published as "A new recombineering system for precise genome-editing in Shewanella oneidensis strain MR-1 using single-stranded oligonucleotides" (Anna D. Corts et al. Nature Scientific Reports. 2019).

In Chapter IV, I show that the CRISPR/Cas9 technology is functional in different Shewanella strains and describe development of a system that couples both recombineering with CRISPR/Cas9. I then demonstrate the extremely high efficiency of this new system for making different DNA changes; small insertions, point mutations and deletions, regardless of the gene or strain to be modified.

Finally, I conclude in Chapter V with a discussion on limitations that should be addressed in order to unlock the full potential of genome engineering in Shewanella, as well as future directions for the technologies described in this dissertation. 


\section{CHAPTER II: \\ DEVELOPMENT AND OPTIMIZATION OF AN \\ EFFICIENT ELECTROTRANSFORMATION METHOD IN \\ SHEWANELLA}

This chapter was adapted from "A new recombineering system for precise genome-editing in Shewanella oneidensis strain MR-1 using single-stranded oligonucleotides", published in Nature Scientific Reports, in 2019 (Anna D. Corts, et al. 2019), and from "Efficient and Precise Genome Editing in Shewanella with Recombineering and CRISPR/Cas9-mediated Counter-selection”, submitted in ACS Synthetic Biology, in 2019.

\subsection{Introduction}

To date, the most reliable method used for introducing DNA into Shewanella spp. has been bacterial conjugation, a somewhat tedious and time-consuming technique when compared to electroporation-based methodologies. Shewanella oneidensis strain MR-1 is a widely used Shewanella strain but displays a low efficiency of electrotransformation for heterologous plasmids derived from different bacterial species, due to its restriction-modification native system (Rachkevych et al. 2014). Since the first attempt of electrotransformation in S. oneidensis in 1994 (Saffarini et al. 1994), only a few studies have used electroporation as a method to transfer DNA in Shewanella (Rachkevych et al. 2014; Myers \& Myers 1997; Zhou \& Ng 2016; Bendall et al. 2013; Shi et al. 2005), and no robust transformation protocol has been established to date.

A recent study in the model organism $E$. coli showed that making electrocompetent cells and performing electroporation at room temperature (Tu et al. 2016) improves DNA transformation up to 10 -fold. Although standard procedure is to keep cells on ice when making electrocompetent cells and during electroporation ( $\mathrm{Wu}$ et al. 2010), we found that preparation of electrocompetent cells at room temperature also increases transformation efficiency in S. oneidensis. Additionally, we optimized a number of other transformation parameters including DNA concentration, cell quantity, growth phase, electroporation conditions and cell competence after freezing at $-80{ }^{\circ} \mathrm{C}$. We found that when using $10 \%$ glycerol as wash buffer, cells grown to stationary phase increases electroporation efficiency by nearly 400 -fold compared to those from early exponential phase, as conventional methods describe. This surprising finding allowed us to make electrocompetent cells 
by simply using overnight cultures which, when frozen at $-80{ }^{\circ} \mathrm{C}$, still maintain the same high transformation efficiency.

We further improved the efficiency to a maximum of $\sim 10^{8}$ transformants/ $\mu \mathrm{g}$ DNA by using $1 \mathrm{M}$ sorbitol as wash buffer and late exponential phase cells and were able to apply this protocol to other Shewanella strains as well.

\subsection{Results}

\subsubsection{Room temperature enhances electrotransformation efficiency in S. oneidensis}

We tested transformation of plasmids pACYC' (a modified version of pACYC184 (Myers \& Myers 1997), 2,703bp) with chloramphenicol (Cm) resistance and pBTBX2 (Prior et al. 2010) $(3,831 \mathrm{bp})$ with kanamycin $(\mathrm{Km})$ resistance, by making electrocompetent cells at room temperature (RT). Each plasmid was purified from the methylation-proficient E. coli UQ950 and from a methylation-minus E. coli (GM1674 or GM2163). The latter contain a mutation in the DNAcytosine methyltransferase $\left(\mathrm{dcm}^{-6}\right)$ that prevents methylation of cytosine residues, which is useful for plasmid purification and transformation into other bacteria because of host modificationdependent restriction systems (Macaluso \& Mettus 1991).

No matter the plasmid type or source, the electroporation efficiency was consistently higher for electrocompetent cells prepared at room temperature (Fig. 2.1a and Fig. 2.2a). In addition, transformation efficiencies were higher when plasmids were purified from the methylation-minus E. coli, as expected.

We observed that cell viability decreased between 4- to 10-fold when making electrocompetent cells on ice (Fig. 2.1b), suggesting cell lysis, as was observed for E. coli (Tu et al. 2016). 
b

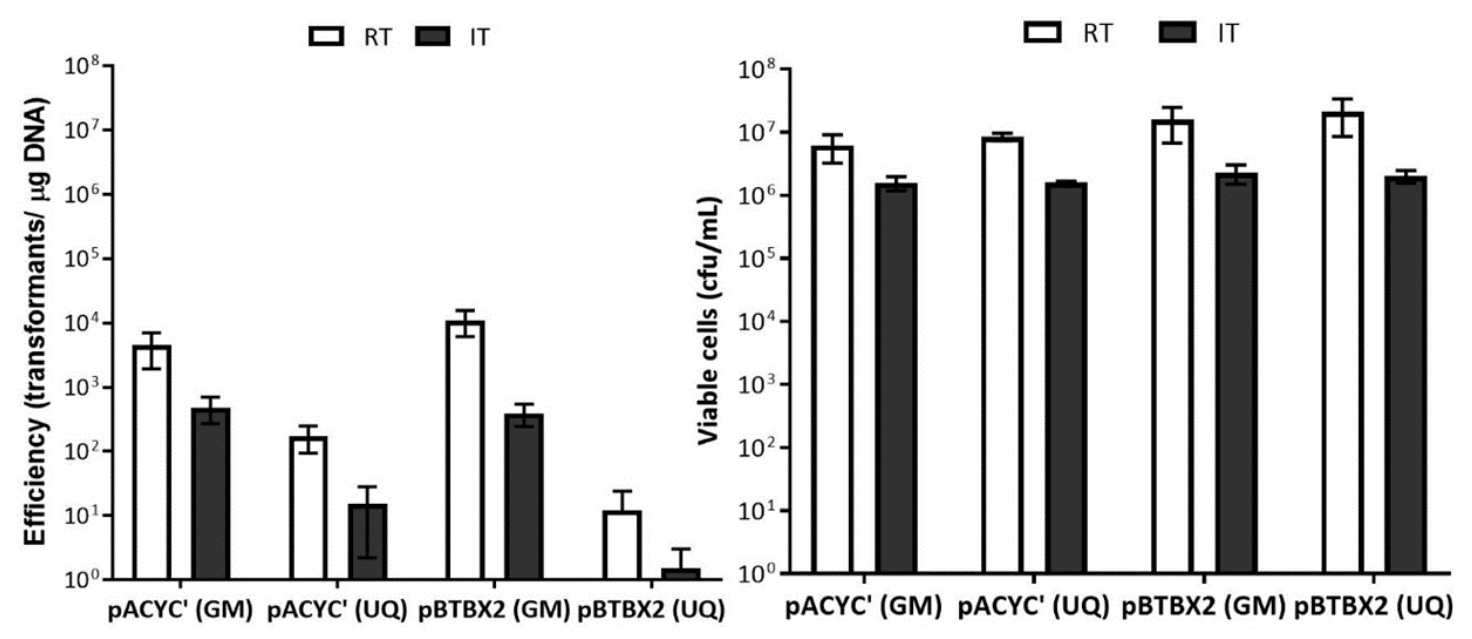

Figure 2. 1. Electrotransformation efficiency and cell viability at room temperature (RT) and ice temperature (IT).

(a) Transformation efficiency. (b) Cell viability. The error bars represent standard error from three independent experiments. Cells were transformed with $250 \mathrm{ng}$ of plasmid pACYC' or pBTBX2 purified from a methylation-minus (GM) or -proficient (UQ) E. coli.

Further analysis by colony PCR and agarose gel electrophoresis of transformants obtained at room temperature indicated successful transformation (Fig. 2.2b). In parallel experiments, mock transformations without plasmid produced no colonies on selective agar plates.
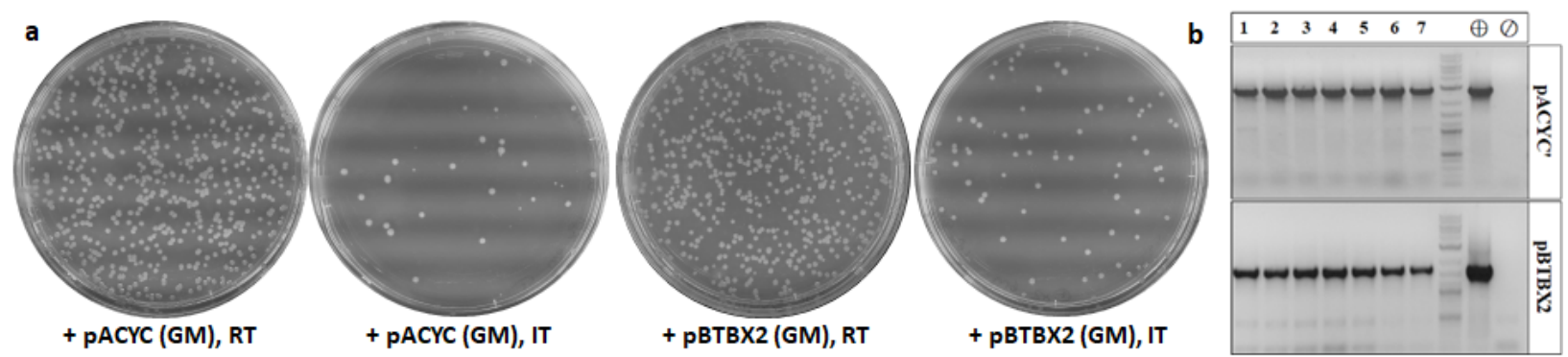

Figure 2. 2. Comparison of electrotransformation at room temperature (RT) and ice temperature (IT).

(a) Cell plates from transformation at RT and IT with each plasmid, pACYC' or pBTBX2, purified from methylation-minus E. coli GM1674 or GM2163 (GM), respectively. One mL of LB medium was added to the samples and the cell suspensions were recovered at $30{ }^{\circ} \mathrm{C}$ for $2 \mathrm{~h}$. After the recovery step, aliquots of cells were not diluted but $900 \mu \mathrm{L}$ of cells (pelleted and suspended in remaining $100 \mu \mathrm{L} \mathrm{LB}$ ) were plated on selective agar plates. (b) Seven transformants from (a) at RT transformation with each plasmid were analyzed by colony PCR, which revealed the presence of the plasmid as expected. $\oplus$ indicates a positive plasmid control, $\oslash$ indicates a negative control $S$. oneidensis with no plasmid. The gel was cropped to show the relevant information and no highcontrast of the image was used. 


\subsubsection{Development of an efficient electroporation method in $S$. oneidensis}

At room temperature the absolute number of transformants increased, but the efficiency was still low. Thus, it was necessary to evaluate other electroporation factors to achieve higher efficiency. We optimized these parameters using the larger plasmid pBTBX2 purified from methylation-minus $E$. coli and further verified our observations using pBTBX2 purified from $E$. coli UQ950 and wild-type $S$. oneidensis.

Cell density and growth phase are essential parameters that can be manipulated to enhance cell transformation efficiency. We investigated the impact of a range of cell densities from early exponential phase to late exponential phase. Notably, higher density resulted in a significant increase in transformation efficiency, up to $\sim 4 \times 10^{6}$ transformants/ $\mu \mathrm{g}$ DNA using overnight cultures at late exponential phase, nearly 400 -fold more transformants than at early exponential phase (Fig. 2.3a). These results are in contrast to E. coli electrotransformation (Wu et al. 2010; Tu et al. 2016; Tu 2005), although in agreement with transformation studies in P. aeruginosa (Choi et al. 2006).

Various amounts of plasmid DNA $(0.01-1.5 \mu \mathrm{g})$ were used to determine the optimal concentration for transformation. As we expected, a linear relationship between plasmid DNA quantity and number of transformants was observed. Specifically, $0.1 \mu \mathrm{g}$ was sufficient for high transformation efficiency (Fig. 2.3b), suggesting that saturation may occur beyond this concentration. Frequency however, resulted in a slight proportional relationship because of a slight decrease in cell viability due to dilution of cells as more DNA is added. Cell number is another parameter that can be manipulated to increase transformation efficiency. Usually, large volumes of cells are used for E. coli transformation(Wu et al. 2010), thus we investigated the effect of using higher quantities of cells by varying the cell volume used per electroporation reaction, from 1 to 9 $\mathrm{mL}$ (Fig. 2.3c). When using one $\mathrm{mL}$ cells, the total viable cells surviving electroporation was only $\sim 1.5 \times 10^{4} \mathrm{cfu} / \mathrm{mL}$ and no transformants were obtained, while in three $\mathrm{mL}, \sim 1.6 \times 10^{7} \mathrm{cfu} / \mathrm{mL}$ viable cells remained after electroporation resulting in the highest efficiency. Increasing the cell volume to 6 and $9 \mathrm{~mL}$, slightly increased the total viable cells but no significant difference in the number of transformants was observed. Frequency always remained constant, $\sim 2 \times 10^{-4}$ transformants/ viable cell, no matter the number of cells surviving.

Various electroporation parameters had been used in earlier $S$. oneidensis transformation attempts (Rachkevych et al. 2014; Myers \& Myers 1997; Zhou \& Ng 2016; Saffarini et al. 1994; Bendall et al. 2013). We found that washing the cells with $\mathrm{dH}_{2} \mathrm{O}$ decreased both the transformation efficiency and frequency, $\sim 80$ - and $\sim 200$-fold, respectively, compared to results using $10 \%$ glycerol. In contrast, $1 \mathrm{M}$ sorbitol and electroporation buffer(Weinstock et al. 2016) significantly 
increased the number of viable cells surviving electroporation, resulting in $\sim 250$ - and $\sim 10$-fold higher efficiency, respectively, than the $10 \%$ glycerol wash and a similar frequency (Fig. 2.3d). The transformation efficiency increased proportionally to the voltage applied, achieving an optimum before decreasing at high voltage. Specifically, $12 \mathrm{kV} / \mathrm{cm}$ gave the best results, $\sim 1 \times 10^{4}$ transformants/ $\mu \mathrm{g}$ DNA (Fig. 2.3e) with a time constant of $\sim 5 \mathrm{~ms}$, which results in a 100- to 3,300fold increase compared to the other voltages applied.

Using the optimized procedure, we examined whether storage at $-80^{\circ} \mathrm{C}$ of late exponential phase electrocompetent cells affected transformation yields. Cells frozen for 30 days maintained as high a transformation efficiency as freshly prepared cells without loss of competence when stored in $10 \%$ glycerol (Fig. 2.3f) or $1 \mathrm{M}$ sorbitol (Fig. 2.4). 
a

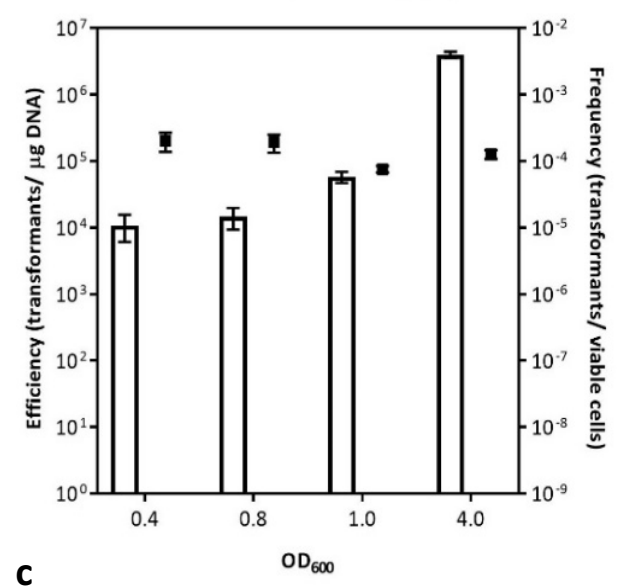

C

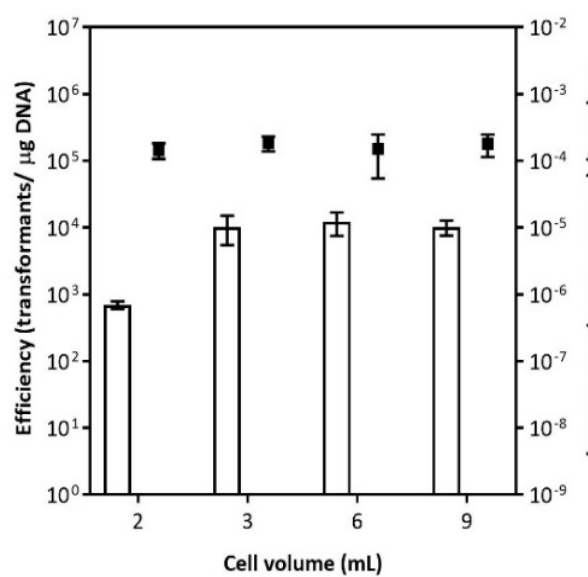

e

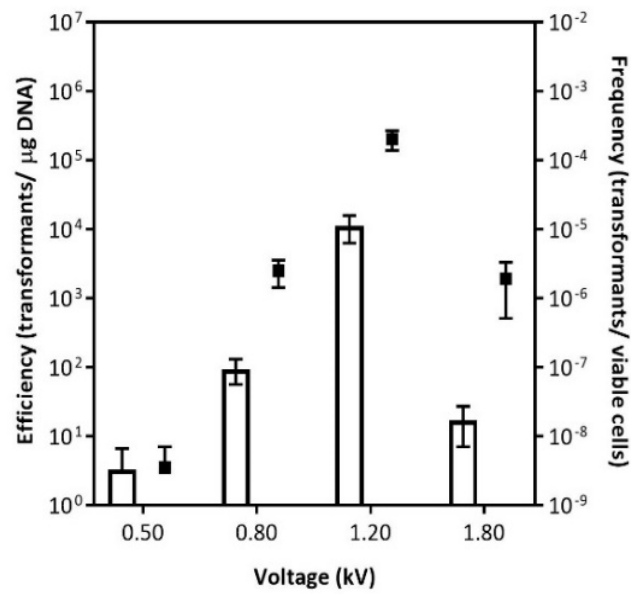

b $\square$ Efficiency - Frequency
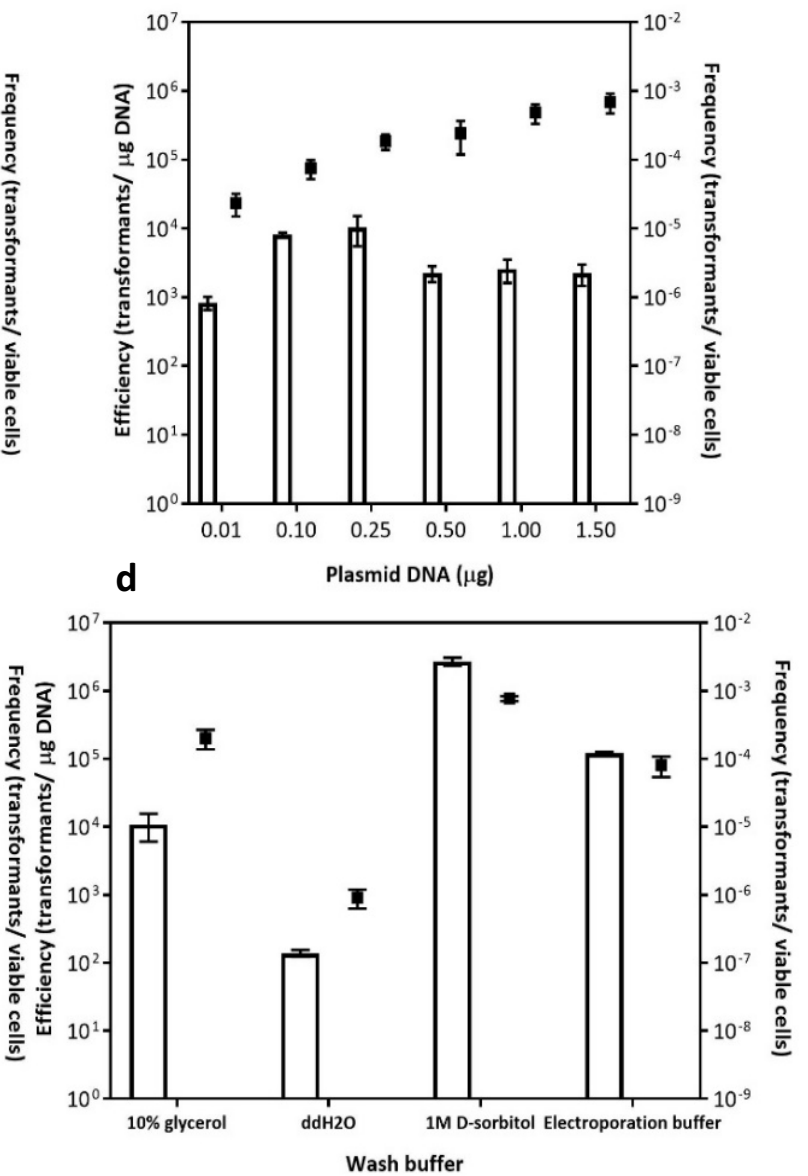

f

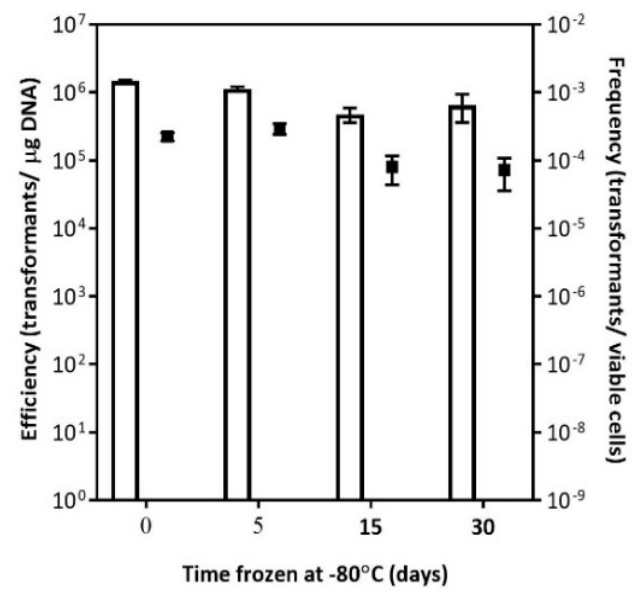

Figure 2. 3. Effects of various parameters on $\boldsymbol{S}$. oneidensis transformation.

(a) Cell density. (b) Plasmid DNA amount. (c) Cell volume. (d) Wash buffer. (e) Voltage. (f) Time frozen in $10 \%$ glycerol at $-80{ }^{\circ} \mathrm{C}$ (one $\mathrm{mL}$ cells was used). Plasmid pBTBX2 purified from methylation-minus E. coli GM2163 was used. The error bars represent standard error from three independent experiments. Three $\mathrm{mL}$ cells were transformed with $250 \mathrm{ng}$ of plasmid DNA, unless otherwise specified. 


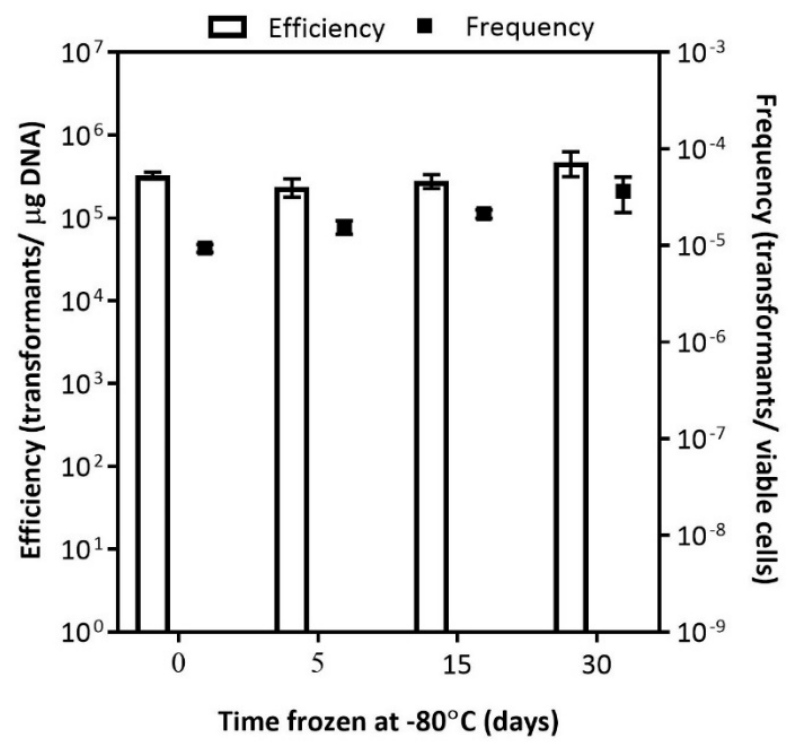

Figure 2. 4. Effect of time frozen in $1 \mathrm{M}$ sorbitol at $-80{ }^{\circ} \mathrm{C}$ on $S$. oneidensis electrotransformation.

One $\mathrm{mL}$ of overnight culture was washed with $1 \mathrm{M}$ sorbitol as described in the Methods section and frozen immediately. At the times specified, cells were tested for electrotransformation with $250 \mathrm{ng}$ of pBTBX2 plasmid DNA isolated from the methylation-minus E. coli strain GM2163. One $\mathrm{mL}$ of LB medium was added to the samples and the cell suspensions were recovered at $30{ }^{\circ} \mathrm{C}$ for $2 \mathrm{~h}$. Aliquots were plated on LB $+\mathrm{Km}$ plates at dilutions to yield single colonies. Transformants were counted after $\sim 36 \mathrm{~h}$ incubation at $30^{\circ} \mathrm{C}$. The error bars represent standard error from three independent experiments.

\subsubsection{Effect of plasmid DNA methylation patterns on electroporation efficiency}

Intrigued by the high efficiencies observed at stationary phase, we tested whether inhibition of the restriction-modification system from the cell was the reason of higher electrotransformation efficiencies at stationary phase cells. For that, we performed electroporation using a plasmid with different methylation patterns; E. coli and Shewanella methylated.

We observed a similar increase in transformants using plasmid purified from E. coli UQ950 and the methylation-deficient E. coli GM2163, 400-fold higher efficiency compared to results with early exponential phase cells. Unexpectedly, when using plasmid DNA purified from $S$. oneidensis (Fig. 2.5), the efficiency only increased $\sim 40$-fold, reaching that of wild-type E. coli MG1655 ( 1 1 10 $0^{4}$ transformants/ $\mu \mathrm{g}$ DNA in our lab, using plasmid pBTBX2 purified from E. coli UQ950).

Surprisingly, higher efficiency of electroporation was obtained with the un-methylated plasmid compared to the $S$. oneidensis methylated plasmid. We think the lower efficiencies observed using $S$. oneidensis purified plasmid may be due to the method for plasmid purification, 
which was initially developed for E. coli, thus may yield lower plasmid amounts when obtained from Shewanella.

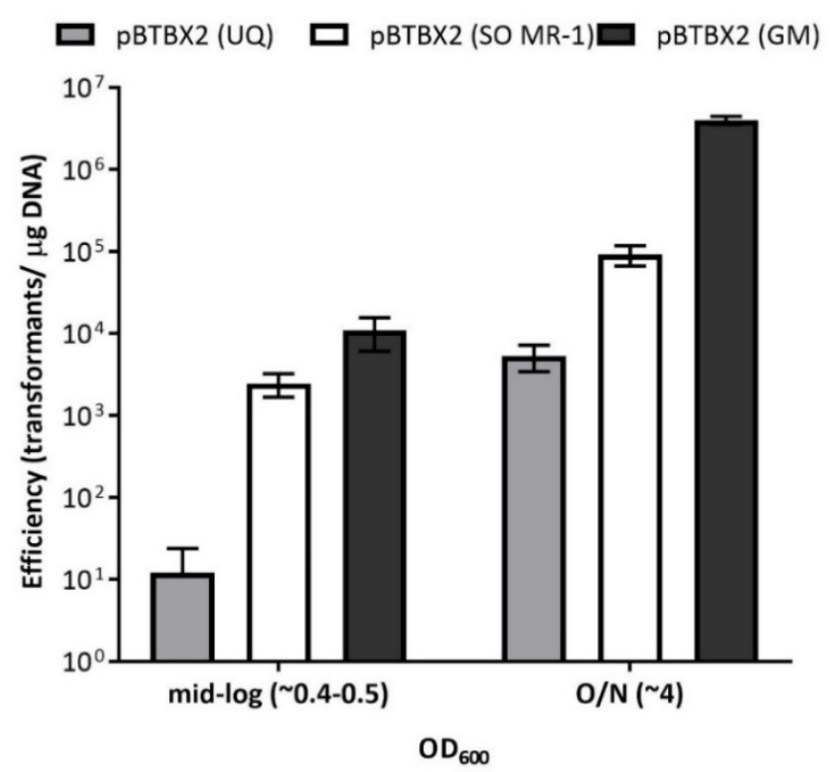

Figure 2. 5. Effect of plasmid DNA methylation patterns on electroporation efficiency. $250 \mathrm{ng}$ of pBTBX2 plasmid DNA purified from methylation-proficient E. coli UQ950, methylation-minus $E$. coli GM2163 or $S$. oneidensis MR-1 was used for electroporation. One mL of LB medium was added to the samples and the cell suspensions were recovered at $30{ }^{\circ} \mathrm{C}$ for $2 \mathrm{~h}$. Aliquots were then plated on $\mathrm{LB}+\mathrm{Km}$ plates at appropriate dilutions to yield single colonies. Transformants were counted after $\sim 36 \mathrm{~h}$ incubation at $30^{\circ} \mathrm{C}$. The error bars represent standard error from three independent experiments.

From this experiment we cannot conclude whether inhibition or changes of the host restriction-methylation system are the logic for the enhanced electroporation efficiency. However, the results indicate that another unknown factor plays a role since the efficiency increases even when the plasmid is methylated with the same host pattern, S. oneidensis.

\subsubsection{Application of electroporation to other Shewanella strains}

We then wished to apply the developed protocol to other Shewanella strains. Given that 1 $\mathrm{M}$ sorbitol resulted in higher number of transformants when using early exponential phase cells, but $10 \%$ glycerol gave a very high efficiency when using stationary phase cells, we first sought to more precisely compare both wash buffers. Thus, we performed electroporation by using cells at different growth phases and made them competent by washing them with $1 \mathrm{M}$ sorbitol or $10 \%$ glycerol (Figure 2.6.) 
Surprisingly, we found that sorbitol gives higher electrotransformation efficiencies up to late exponential phase when cells are at an $\mathrm{OD}_{600}$ of $\sim 2$, resulting in a maximum efficiency of $\sim 10^{8}$ transformants $\mu \mathrm{g}$ DNA and a frequency of one transformant every $\sim 200$ viable cells. This efficiency numbers were notable as it results in a 100-fold increase compared to the glycerol washed cells and 300-fold higher than the transformants obtained with wild-type E. coli. At stationary phase cells, however, the same number of transformants is obtained for both the glycerol and sorbitol washes.

Using the same kanamycin resistant plasmid, pBTBX2, successful electrotransformation was obtained when using S. amazonensis SB2B cells washed with sorbitol, obtaining an efficiency of $\sim 10^{6}$ transformants/ $\mu \mathrm{g}$ DNA. Interestingly, SB2B cells behaved differently than MR-1 cells, the efficiency was maintained the same up to $\mathrm{OD}_{600} \sim 2$, but decreased 100 -fold when using stationary phase cells (Fig. 2.6). SB2B cells were verified by colony PCR and mock electroporations with no plasmid DNA showed kanamycin resistant colonies as expected.

We were also able to obtain kanamycin resistant Shewanella sp. W3-18-1 cells when performing the electroporation method using sorbitol as wash buffer, albeit at much lower efficiencies (Fig. 2.6). Curiously, although mock transformations resulted in no transformants on kanamycin plates, we were unable to PCR verify all the transformant colonies picked from the selection plates. Some colonies resulted in the right PCR product from amplification of a region in the plasmid backbone, while others showed no PCR amplification. Repeated attempts by colony PCR and PCR from purified plasmid resulted in the same outcome. Further experiments would need to be done to solve this ambiguity with this strain. 


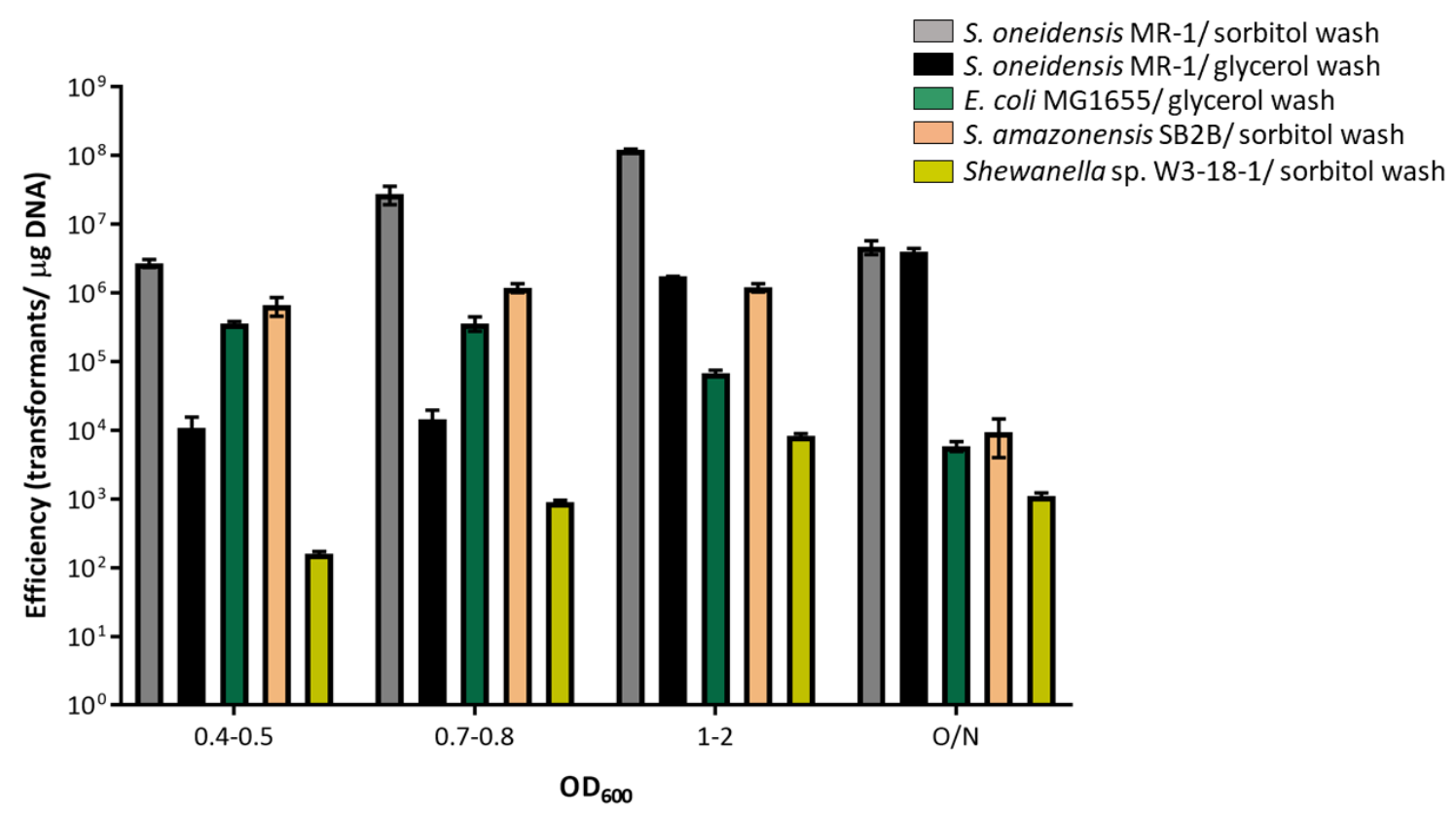

Figure 2. 6. Electroporation efficiencies in different Shewanella strains compared to E. coli. $250 \mathrm{ng}$ of pBTBX2 purified from E. coli GM2163 was used for electroporation. One $\mathrm{mL}$ of LB medium was added to the samples and cell suspensions were recovered at $30^{\circ} \mathrm{C}$ (Shewanella) or $37^{\circ} \mathrm{C}$ (E. coli) for $2 \mathrm{~h}$. Aliquots were plated on $\mathrm{Km}$ plates at appropriate dilutions to yield single colonies. Transformants were counted after $\sim 36 \mathrm{~h}$ (Shewanella) or $\sim 20 \mathrm{~h}$ (E. coli) incubation. The error bars represent standard error from three independent experiments.

Below we describe in detail all the steps of our electroporation protocol, for obtaining the highest transformation efficiency in the model Shewanella strain S. oneidensis MR-1, which is also functional in S. amazonensis SB2B. 
Table 2. 1. Gralnick Lab high efficiency electroporation protocol for plasmid transformation in S. oneidensis MR-1.

\begin{tabular}{|c|c|c|}
\hline STEP & DESCRIPTION & NOTES \\
\hline $\begin{array}{l}\text { 1. Preliminary } \\
\text { Steps }\end{array}$ & $\begin{array}{l}\text { a) Culture a colony overnight in } \sim 5 \mathrm{~mL} \text { LB } \\
\text { liquid medium in a } 20 \mathrm{~mL} \text { culture tube and at } \\
30^{\circ} \mathrm{C} \text { with shaking }(\sim 200 \mathrm{rpm}) \\
\text { b) Make } 1 \mathrm{M} \text { sorbitol pH } 7.6 \\
\text { c) Obtain purified plasmid DNA (if possible, } \\
\text { preferably from a methylation-minus } E \text {. coli) }\end{array}$ & $\begin{array}{l}* \text { DNA concentration should be } \\
\sim 100 \mathrm{ng} / \mu \mathrm{L} \text {. Use a Nanodrop if } \\
\text { available; if } 260 / 230 \text { and } 280 / 260 \\
\text { ratios are not } \sim 2 \text { and } \sim 1.8, \\
\text { respectively, the efficiency may be } \\
\text { lower }\end{array}$ \\
\hline $\begin{array}{l}\text { 2. Making } \\
\text { electrocompetent } \\
\text { cells at room } \\
\text { temperature }\end{array}$ & $\begin{array}{l}\text { a) Subculture cells to a starting } \mathrm{OD}_{600} \text { of } \\
0.08 \\
\text { b) Prepare } \sim 100 \text { ng plasmid DNA in a } 2 \mathrm{~mL} \\
\text { Eppendorf tube. Perform a control with no } \\
\text { DNA } \\
\text { b) Once cells are at } \mathrm{OD}_{600} \sim 2 \text {, collect } 3 \mathrm{~mL} \text {, } \\
1 \mathrm{~mL} \text { per Eppendorf tube, spin at } 7,906 \mathrm{rcf} \\
\text { for } 1 \mathrm{~min} \\
\text { c) Combine the three pellets into one by } \\
\text { washing with } 1 \mathrm{~mL} 1 \mathrm{M} \text { sorbitol, and spin } \\
\text { again } \\
\text { d) Wash cells for a total of three times with } \\
1 \mathrm{M} \text { sorbitol and spinning at } 7,906 \text { rcf for } 1 \\
\text { min } \\
\text { e) After the third wash, decant the sorbitol } \\
\text { and leave } \sim 60-70 \mu \mathrm{L} \text { for suspending the } \\
\text { cells } \\
\text { f) Suspend cells gently by shaking the tube } \\
\text { and pipetting with P100, then mix cells with } \\
\text { the DNA by swirling around with the tip }\end{array}$ & $\begin{array}{l}\text { *When suspending cells with } \\
\text { sorbitol, be gentle pipetting with } \\
\text { P1000 10 times } \\
* \text { When decanting sorbitol after } \\
\text { spins, use care to not lose the } \\
\text { pellet; gently tap the tube } 3 \text { times } \\
* \text { A } 0.1 \mathrm{~cm} \text { cuvette fits } \sim 100 \mu \mathrm{L} \\
\text { max. A } 0.2 \mathrm{~cm} \text { cuvette requires } \\
\text { higher voltages } \\
* \text { If desired, use } 10 \% \text { glycerol to } \\
\text { freeze the cells at }-80^{\circ} \mathrm{C} \text {, as } \\
\text { glycerol is a better buffer to } \\
\text { maintain cells competent (Corts et } \\
\text { al. 2019) }\end{array}$ \\
\hline $\begin{array}{l}\text { 3. Performing } \\
\text { electroporation } \\
\text { at room } \\
\text { temperature }\end{array}$ & $\begin{array}{l}\text { a) Transfer the mixture immediately into a } \\
0.1 \mathrm{~cm} \text { cuvette without making bubbles } \\
\text { b) Electroporate at } 1.2 \mathrm{kV} \text {. Time constant } \\
\text { (tc) should be } \sim 4 \\
\text { c) Quickly add } 1 \mathrm{~mL} \text { LB into the cuvette and } \\
\text { mix gently while pipetting up and down } \\
\text { d) Transfer the cells into the same } 2 \mathrm{~mL} \text { tube } \\
\text { you had the DNA in } \\
\text { e) Let the cells recover at } 30^{\circ} \mathrm{C} \text { for } 2 \mathrm{~h} \text { with } \\
\text { shaking } \sim 200 \mathrm{rpm}\end{array}$ & $\begin{array}{l}* \text { Electroporator settings: } 10 \mu \mathrm{F}, \\
600 \Omega \text { (if different, voltage may } \\
\text { need to be optimized) } \\
* \text { If tc is not } \sim 4 \text {, efficiency may be } \\
\text { lower }\end{array}$ \\
\hline 4. Plating & $\begin{array}{l}\text { a) Make 10x serial dilutions in LB } \\
\text { b) Plate several different dilutions } \\
\text { c) Incubate plates at } 30^{\circ} \mathrm{C} \text { for } \sim 24-36 \mathrm{~h} \text { until } \\
\text { colonies appear }\end{array}$ & $\begin{array}{l}\text { * Depending on the source of the } \\
\text { DNA, efficiency varies(Corts et al. } \\
2019 \text { ). Maximum efficiency } \\
\text { obtained here is } \sim 10^{8} \\
\text { transformants/ } \mu \mathrm{g} \text { DNA, which is } 1 \\
\text { transformant every } \sim 200 \text { viable } \\
\text { cells (Fig. S1) } \\
\text { * No colonies should appear on the } \\
\text { control with no DNA (if the cells } \\
\text { are not diluted enough, false } \\
\text { positives can appear on a lawn of } \\
\text { dead cells, depending on the } \\
\text { antibiotic used) }\end{array}$ \\
\hline
\end{tabular}




\subsection{Discussion}

The ability to transfer exogenous DNA into cells is essential for genetic engineering of microorganisms. Although conjugation has been widely used as means to transfer plasmid DNA in bacteria, electroporation is more convenient for large-scale studies and allows transfer of both circular and linear DNA molecules.

The simple and highly efficient electrotransformation protocol reported in this study provides an opportunity to more easily manipulate $S$. oneidensis. Key features of this electroporation procedure are using small quantities of cells, using cells at late exponential phase, washing with $1 \mathrm{M}$ sortbiol and making electrocompetent cells and performing electroporation at room temperature to avoid lysis. With these conditions, $\sim 10^{8}$ transformants $/ \mu \mathrm{g}$ DNA were obtained using a non-methylated plasmid.

For a simple plasmid transformation, stationary phase cells from an overnight culture can also be used, producing $\sim 10^{6}$ transformants/ $\mu$ g DNA with either $10 \%$ glycerol or $1 \mathrm{M}$ sorbitol, which efficiency was maintained when cells were frozen for long term storage at $-80{ }^{\circ} \mathrm{C}$ (up to a month tested). We were surprised by the high efficiency achieved under this condition compared to early exponential phase, as this does not replicate across different bacteria (Tu et al. 2016; Wu et al. 2010; Tu 2005), although it is in agreement with P. aureginosa procedures (Choi et al. 2006). We do not know why this difference in preferred growth phase prior to electroporation. We suspect it is not due simply to a higher input of viable cells, but possibly also to a reduced level of host nuclease activity in stationary phase and other unknown factors.

\subsection{Materials and Methods}

\subsubsection{Bacterial Strains, plasmids and culture conditions}

All strains and plasmids used in this study are listed in Table S1. Shewanella was routinely grown in LB liquid medium or on LB agar at $30^{\circ} \mathrm{C}$. When needed, culture media was supplemented with chloramphenicol $(\mathrm{Cm}, 6.5 \mu \mathrm{g} / \mathrm{mL})$, kanamycin $(\mathrm{Km}, 50 \mu \mathrm{g} / \mathrm{mL})$ or spectinomycin (Spect, 50 $\mu \mathrm{g} / \mathrm{mL}$ ). Plasmid pACYC' was isolated from E. coli UQ950 and from methylation-minus E. coli GM1674, while plasmid pBTBX2 from UQ950 and methylation-minus E. coli GM2163. 
Table 2. 2. Strains and plasmids used in this work.

\begin{tabular}{|c|c|c|}
\hline Strain or plasmid & Description & Reference or source \\
\hline \multicolumn{3}{|l|}{ Strain } \\
\hline JG274 & S. oneidensis MR-1, wild type & Gralnick Lab \\
\hline JG239 & Shewanella sp. W3-18-1 & Gralnick Lab \\
\hline JG2150 & S. oneidensis MR-1, lacZ & Gralnick Lab \\
\hline JG98 & S. amazonensis SB2B & Gralnick Lab \\
\hline UQ950 & E. coli $\mathrm{DH} 5 \alpha \lambda$ (pir) host for cloning & (Saltikov \& Newman 2003) \\
\hline GM2163 & E. coli methylation-minus, $\mathrm{CmR}$ & CGSC\#: 6581 \\
\hline GM1674 & E. coli methylation-minus & CGSC\#7971 \\
\hline MG1655 & E. coli F- lambda- $i l v G-r f b-50 r p h-1$ & Gralnick Lab \\
\hline \multicolumn{3}{|l|}{ Plasmid } \\
\hline pACYC' & Modified pACYC184, p15a ori, CmR & (Myers \& Myers 1997) \\
\hline pBTBX2 & pBBR1 ori, $\mathrm{KmR}, \mathrm{pBAD}$ & (Prior et al. 2010) \\
\hline
\end{tabular}

\subsubsection{DNA manipulations}

Plasmid DNAs were isolated using Invitrogen Plasmid mini Kit. The pACYC' plasmid is a modified version of pACYC1 84 (Myers \& Myers 1997) harboring the chloramphenicol resistance gene $(\mathrm{cmR})$; the tetracycline resistance gene $($ tet $R)$ was removed by inserting the oriT RP4 in its place, since tet $R$ shared $94 \mathrm{bp}$ homology to another region in the plasmid backbone, the promoter of $\mathrm{cmR}$ was replaced by the promoter of $t e t R$ and, nucleotides $442-586$ were removed due to $145 \mathrm{bp}$ shared homology to the E. coli MG1655 genome.

All plasmids listed in Table 2.2 were created using Gibson Assembly Ultra Kit (Synthetic Genomics), except for removal of nucleotides 442-586 in pACYC184, which was performed by blunt ligation using NEB T4 DNA ligase. Primers listed in Table 2.3 were used to create linear dsDNA by PCR amplification in a $50 \mu \mathrm{L}$ reaction with Q5 polymerase. The linear dsDNA vectors were DpnI digested for at least 2 hours and gel-purified. Inserts were amplified in a $50 \mu \mathrm{L}$ reaction with Q5 polymerase and PCR purified. DNA fragments were assembled at a ratio 1/1 following supplier instructions and transferred in chemically competent $E$. coli UQ950. Cells were recovered for $\sim 2 \mathrm{~h}$ in LB medium, plated on selective agar plates and incubated at $37^{\circ} \mathrm{C}$.

For colony PCR, a single colony was transferred to $25 \mu \mathrm{LdH} \mathrm{d}_{2} \mathrm{O}$, boiled at $95{ }^{\circ} \mathrm{C}$ for $5 \mathrm{~min}$ and frozen at $-80^{\circ} \mathrm{C}$ for $5 \mathrm{~min}$. The cell debris was removed by centrifugation for $1 \mathrm{~min}$ and $2 \mu \mathrm{L}$ of the supernatant were used as a source of template in a $20 \mu \mathrm{L}$ reaction. GoTaq $2 X$ MM (Promega) was used and supplier instructions were followed. After PCR, $10 \mu \mathrm{L}$ aliquots were analyzed by agarose gel electrophoresis. 
Table 2. 3. Primers used in this study.

\begin{tabular}{|c|c|c|}
\hline Name & Sequence $\left(5^{\prime}->3^{\prime}\right)^{a}$ & Description \\
\hline \multicolumn{3}{|c|}{ Primers for plasmid modification of pACYC184 (Myers \& Myers 1997) } \\
\hline ADC143 & TATGGGGCTGACTTCAGGTGC & $\begin{array}{l}\text { Rev primer to linearize } \mathrm{pACYC} 184 \text { for } \\
\text { cloning oriT }\end{array}$ \\
\hline $\mathrm{ADC} 144$ & TAACGGATTCACCACTCCAAG & $\begin{array}{l}\text { For primer to linearize pACYC } 184 \text { for } \\
\text { cloning oriT }\end{array}$ \\
\hline $\mathrm{ADC} 145$ & $\begin{array}{l}\text { gcacctgaagtcagccccataTGTAGACTT } \\
\text { TCCTTGGTGTATCCAACG }\end{array}$ & $\begin{array}{l}\text { For primer for cloning oriT RP4 in } \\
\text { pACYC184 }\end{array}$ \\
\hline ADC146 & $\begin{array}{l}\text { cttggagtggtgaatccgttaGAATAAGGG } \\
\text { ACAGTGAAGAAGGAACAC }\end{array}$ & $\begin{array}{l}\text { Rev primer for cloning oriT RP4 in } \\
\text { pACYC184 }\end{array}$ \\
\hline ADC147 & $\begin{array}{l}\text { GCAGACCAAAACGATCTCAAG } \\
\text { AAG }\end{array}$ & $\begin{array}{l}\text { For primer to sequence verify oriT RP4 } \\
\text { cloning }\end{array}$ \\
\hline ADC158 & $\begin{array}{l}\text { caacttttggcgaaaatgagacgtCGATATA } \\
\text { AGTTGTAATTCTCATGTTTGAC } \\
\text { AGCTT }\end{array}$ & For primer to amplify the tet $R$ promoter \\
\hline ADC159 & $\begin{array}{l}\text { tggtatatccagtgatttttttctccatACACGGT } \\
\text { GCCTGACTGC }\end{array}$ & Rev primer to amplify the tet $R$ promoter \\
\hline ADC160 & $\begin{array}{l}\text { ATGGAGAAAAAAATCACTGGA } \\
\text { TATACCA }\end{array}$ & $\begin{array}{l}\text { For primer to linearize pACYC184 for } \\
\text { cloning } \mathrm{P}_{\text {tetR }}\end{array}$ \\
\hline ADC161 & $\begin{array}{l}\text { ACGTCTCATTTTCGCCAAAAGT } \\
\text { TG }\end{array}$ & $\begin{array}{l}\text { Rev primer to linearize } \mathrm{pACYC} 184 \text { for } \\
\text { cloning } \mathrm{P}_{\text {tetR }}\end{array}$ \\
\hline $\mathrm{ADC} 170$ & $\begin{array}{l}\text { gegaagtgatcttccgtcacagGGCTTACT } \\
\text { ATGTTGGCACTGATGAG }\end{array}$ & $\begin{array}{l}\text { For primer to linearize } \mathrm{pACYC} 184 \text { to } \\
\text { remove nucleotides } 442-586 \text {, creating } \\
\text { pACYC' }\end{array}$ \\
\hline ADC171 & $\begin{array}{l}\text { ctcatcagtgccaacatagtaagccCTGTGAC } \\
\text { GGAAGATCACTTCGC }\end{array}$ & $\begin{array}{l}\text { Rev primer to linearize } \mathrm{pACYC} 184 \text { to } \\
\text { remove nucleotides } 442-586 \text {, creating } \\
\text { pACYC' }\end{array}$ \\
\hline ADC172 & $\begin{array}{l}\text { GGCTTACTATGTTGGCACTGAT } \\
\text { GAG }\end{array}$ & For primer to blunt ligate $\mathrm{pACYC}$ \\
\hline $\mathrm{ADC} 173$ & CTGTGACGGAAGATCACTTCGC & Rev primer to blunt ligate $\mathrm{pACYC}$ \\
\hline $\mathrm{ADC} 174$ & CCCGTTCGTAAGCCATTTCC & $\begin{array}{l}\text { For primer to sequence verify removal of } \\
\text { nucleotides } 442-586\end{array}$ \\
\hline \multicolumn{3}{|c|}{ Primers for plasmid analysis } \\
\hline $\mathrm{ADC} 44$ & GTTTCACTTGATGCTCGATGAG & $\begin{array}{l}\text { For primer to check for the presence of } \\
\text { plasmid pBTBX2 in the cells }\end{array}$ \\
\hline $\mathrm{ADC} 105$ & AATCCCTGTGGTCAAGCTCG & $\begin{array}{l}\text { Rev primer to check for the presence of } \\
\text { plasmid pBTBX2 in the cells }\end{array}$ \\
\hline $\mathrm{ADC} 10$ & $\begin{array}{l}\text { CAGGCATTTGAGAAGCACACG } \\
\text { G }\end{array}$ & $\begin{array}{l}\text { For primer to check for the presence of } \\
\text { plasmid } p A C Y C^{\prime} \text { in the cells }\end{array}$ \\
\hline ADC9 & GCGATGCAGATCCGGAACAT & $\begin{array}{l}\text { Rev primer to check for the presence of } \\
\text { plasmid pACYC' in the cells }\end{array}$ \\
\hline
\end{tabular}

${ }^{\mathrm{a}}$ Lower case designates Gibson assembly overhangs.

\subsubsection{Preparation of competent cells and electrotransformation}

Shewanella cells were made competent by using a microcentrifuge-based procedure similar to that of P. aeruginosa (Choi et al. 2006). Overnight cultures were diluted to an $\mathrm{OD}_{600}$ of 0.08 and, 
once the cells reached an $\mathrm{OD}_{600}$ between 0.4-0.5 ( 4x10 $\left.{ }^{8} \mathrm{CFU} / \mathrm{mL}\right)$ (unless otherwise specified), three $\mathrm{mL}$ of cells were used per electroporation reaction. One $\mathrm{mL}$ was distributed per eppendorf tube and the cells were harvested at room temperature for one min at $7607 \mathrm{rcf}$. One $\mathrm{mL}$ of room temperature $10 \%$ glycerol or $1 \mathrm{M}$ sorbitol was used to wash and combine three cell pellets into one. The cell pellets were washed two more times and suspended in the residual $\sim 50 \mu \mathrm{L}$, which resulted on average $10^{6}-10^{7}$ viable bacteria after electroporation. When overnight cultures were used, the washing steps were performed at $7607 \mathrm{rcf}$ for two min. If competent cells were to be stored, cells were immediately frozen on liquid nitrogen and transferred to $-80{ }^{\circ} \mathrm{C}$. When using frozen cells for electrporation, a 10 min defrost was allowed on ice followed by three min incubation with the plasmid DNA at room temperature prior to electroporation.

For electroporation, $250 \mathrm{ng}$ of plasmid was mixed with $\sim 50 \mu \mathrm{L}$ of competent cells and the mixture was transferred to a room temperature $0.1 \mathrm{~cm}$ cuvette. After applying a pulse (settings: 10 $\mu \mathrm{F}, 600 \Omega, 1.2 \mathrm{kV}$ on a Bio Rad MicroPulser ${ }^{\mathrm{TM}}$ ), one $\mathrm{mL}$ of room temperature liquid $\mathrm{LB}$ was added and transferred into a two $\mathrm{mL}$ Eppendorf tube for greater aeration. Cells were recovered at $30^{\circ} \mathrm{C}$ shacking for two hours prior to plating on agar plates with antibiotics. The plates were incubated at $30^{\circ} \mathrm{C}$ until colonies appeared. To determine number of viable cells, $10 \mu \mathrm{L}$ of a range of 10 -fold serial dilutions were spotted onto LB agar. To determine the number of transformants, $100 \mu \mathrm{L}$ of the aliquots were plated on selective plates at appropriate dilutions to yield single colonies. For the lower efficiency conditions, the remaining cells (pelleted at 6,010 rcf for three min and suspended in remaining $100 \mu \mathrm{L} \mathrm{LB}$ ) were also plated.

For the ice-chilled electrotransformation, competent cells were kept on ice for $15 \mathrm{~min}$ and then washed with cold 10\% glycerol while keeping the tubes on ice. Electroporation was done with ice-chilled cuvettes. 


\title{
CHAPTER III:
}

\section{A NEW RECOMBINEERING SYSTEM FOR PRECISE GENOME-EDITING IN SHEWANELLA USING SINGLE- STRANDED OLIGONUCLEOTIDES}

\begin{abstract}
This chapter was adapted from "A new recombineering system for precise genome-editing in Shewanella oneidensis strain MR-1 using single-stranded oligonucleotides", published in Nature Scientific Reports, in 2019 (Anna D. Corts, et al. 2019), and from "Efficient and Precise Genome Editing in Shewanella with Recombineering and CRISPR/Cas9-mediated Counter-selection", submitted to ACS Synthetic Biology, on April 2019.
\end{abstract}

\subsection{Introduction}

Developing a high efficiency method for DNA uptake in S. oneidensis has enabled us to introduce single-stranded DNA oligonucleotides (oligos) to perform in vivo homologous recombination-mediated genetic engineering, known as recombineering, a powerful tool for precise DNA editing developed in E. coli. Recombineering enables efficient and rapid in vivo construction of mutant alleles by taking advantage of homologous recombination mediated by expression of bacteriophage proteins, such as the Red system from phage $\lambda$ and the RecET system from the Rac prophage. Linear DNA fragments, either double-stranded DNA (dsDNA) or singlestranded DNA (ssDNA) can be designed with short homology sequences, as short as 50 base pairs (bp), to a genomic target allowing accurate insertion, deletion or alteration of any DNA sequence without relying on conveniently located restriction sites (Sharan et al. 2009; Boyle et al. 2013; Sawitzke et al. 2011).

The $\lambda$ Red system includes three proteins: Exo, Beta, and Gam. Exo is an exonuclease, which degrades DNA in the 5' to 3' direction creating 3' ssDNA overhangs; the recombinase Beta is a single-strand annealing protein, which binds to these ssDNA overhangs and pairs them with complementary ssDNA targets (Murphy 1998). Exo and Beta are functionally equivalent although not related at the sequence level to RecE and RecT, respectively. The Gam protein inhibits the RecBCD and SbcCD nuclease activities in the host, protecting the exogenous DNA from being degraded (Murphy 1991). The Rac prophage does not encode a known analogue of Gam, but a study showed it can be combined with RecET (Zhang et al. 1998). Recombineering with dsDNA 
requires the presence of both exonuclease and recombinase (Datta et al. 2008), but ssDNA recombineering only requires the expression of the recombinase (Ellis et al. 2001).

Adapting recombineering for a new species is challenging; however, because the $\lambda$ Red and RecET systems do not necessarily maintain high efficiency across different bacteria (Huang et al. 2017; Hossain et al. 2015; Hu et al. 2014), there may be a dependence on host-specific machinery (Datta et al. 2008; Muyrers et al. 2000). Since recombineering was first applied in $E$. coli (Datsenko \& Wanner 2000; Baba et al. 2006), a few other phage homologous recombination systems have been found to promote recombination in Pseudomonas, Vibrio, Lactobacillus, Mycobacteria, Photorhabdus and Staphylococcus (van Kessel \& Hatfull 2007; Yin et al. 2015; Yang et al. 2015; Swingle, Bao, et al. 2010; Oh \& Van Pijkeren 2014; Aparicio et al. 2018; Penewit et al. 2018; Lee et al. 2017; Datta et al. 2008), and we have explored whether this technology can be established in Shewanella. To maximize the recombination efficiency, we identified $\lambda$ Red recombinase homologs in Shewanella species and have tested a system from Shewanella sp. W318-1 (Yang et al. 2013). Here, we demonstrate the use of Shewanella sp. W3-18-1 recombinase, which shares $55 \%$ identity to the $\lambda$ Red Beta recombinase from $E$. coli phage $\lambda$, for targeted mutagenesis using ssDNA in S. oneidensis as well as S.amazonensis. This recombinase is also active in wild type E. coli MG1655, achieving higher levels of recombination than RecT and comparable to $\lambda$ Red Beta. This recombineering process, outlined in Fig. 3.1, can increase the ease with which complex genome engineering efforts are completed in Shewanella. In contrast to other mutagenesis methods, this technology allows precise editing at the single nucleotide level in a few days. 


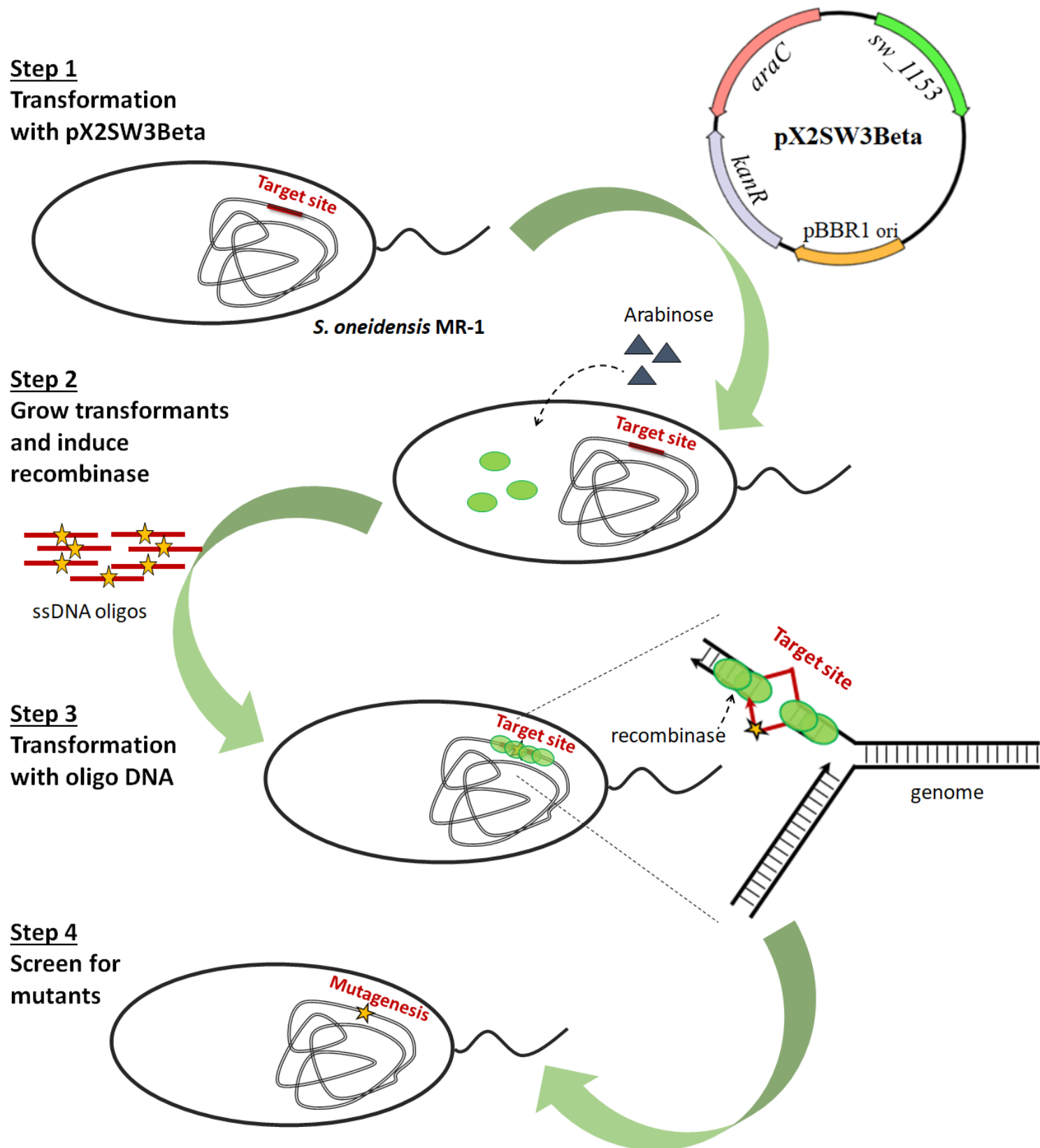

Figure 3. 1. Representation of the stepwise approach used for recombineering in $\mathbf{S}$. oneidensis. The system consists of one plasmid, pX2SW3Beta, expressing the recombinase W3 Beta $\left(s w_{-} 1153\right)$ from the inducible arabinose promoter which is regulated by the arabinose repressor $(\operatorname{araC})$. The plasmid is introduced into host cells by electrotransformation, followed by plating on $\mathrm{LB}+\mathrm{Km}$ and growth at $30^{\circ} \mathrm{C}$. The recombinase resident in the resulting strain is induced with arabinose and the cells are made electrocompetent. ssDNA oligos are transferred by electroporation to generate targeted mutations. After recovery, the cells are plated on LB $+\mathrm{Km}$ and incubated at $30^{\circ} \mathrm{C}$. Mutants are then screened by PCR and the process can be repeated. 


\subsection{Results}

\subsubsection{W3 Beta from Shewanella sp. W3-18-1 is homologous to $\lambda$-Red Beta}

First, we tested for any toxicity effect when expressing the recombinases under the arabinose (ara) inducible pBAD promoter in the pBTBX2 plasmid. Unexpectedly, $\lambda$ Red Beta caused a significant growth defect when expressed in $S$. oneidensis at low levels of induction of 1 $\mathrm{mM}$ ara. No change in growth was observed when expressing RecT up to $5 \mathrm{mM}$ ara (Fig. 3.2).

We also searched for a new recombinase from a native Shewanella phage and identified a potential $\lambda$ Red Beta homolog (Fig. 3.3) in Shewanella sp. W3-18-1, termed W3 Beta (NCBI accession WP_011788511, locus Sputw3181_1153, annotated herein as sw_1153), which did not adversely affect cell growth when induced (Fig. 3.2) . 
a

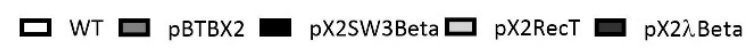

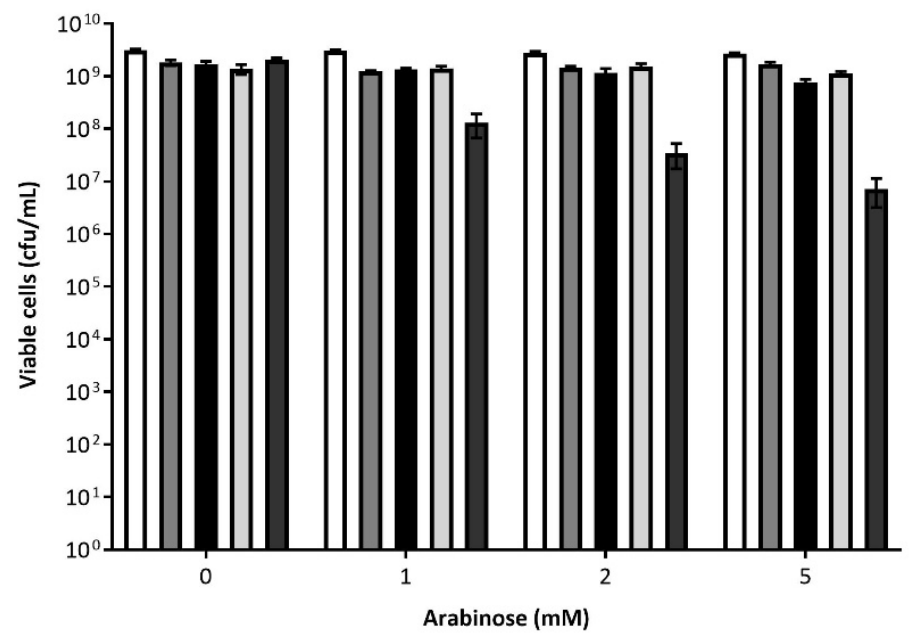

b

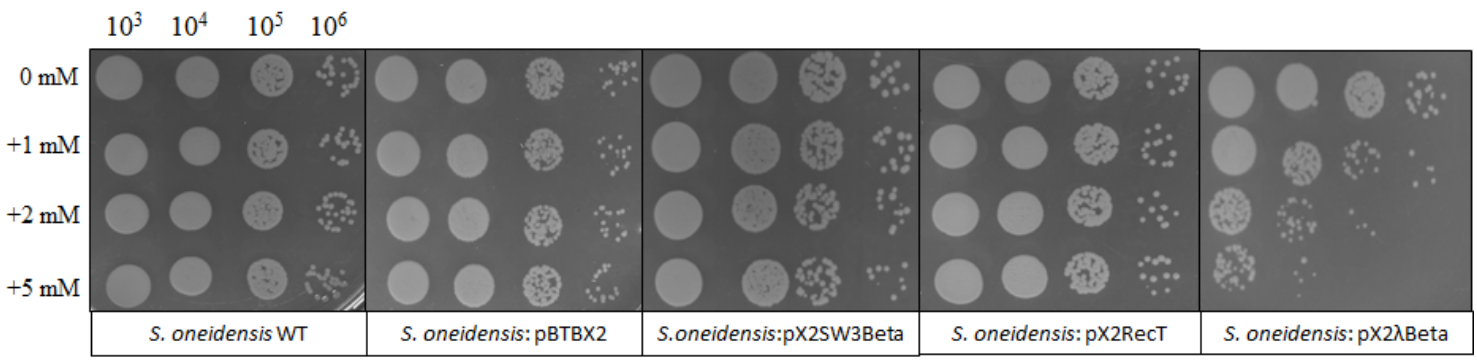

Figure 3. 2. Effect of recombinase expression on $S$. oneidensis growth.

(a) Effect of varying arabinose concentration on viability of wild-type cells (WT), cells carrying the empty vector pBTBX2 and cells with the vector expressing the recombinase under pBAD (W3 Beta, RecT or $\lambda$ Red Beta). (b) Colony forming units from a range of 10 -fold serial dilutions. Cells were subcultured to a starting $\mathrm{OD}_{600}$ of 0.08 and grown to an $\mathrm{OD}_{600}$ of $\sim 0.4-0.5$ and then, the specified arabinose concentration was added. Cells were induced for $3 \mathrm{~h}$ and after induction, aliquots were spotted onto LB agar (WT cells) or LB $+\mathrm{Km}$ (cells with plasmid) at different dilutions. Colonies were counted after $\sim 24 \mathrm{~h}$ incubation at $30^{\circ} \mathrm{C}$. The error bars represent standard error from three independent experiments. 


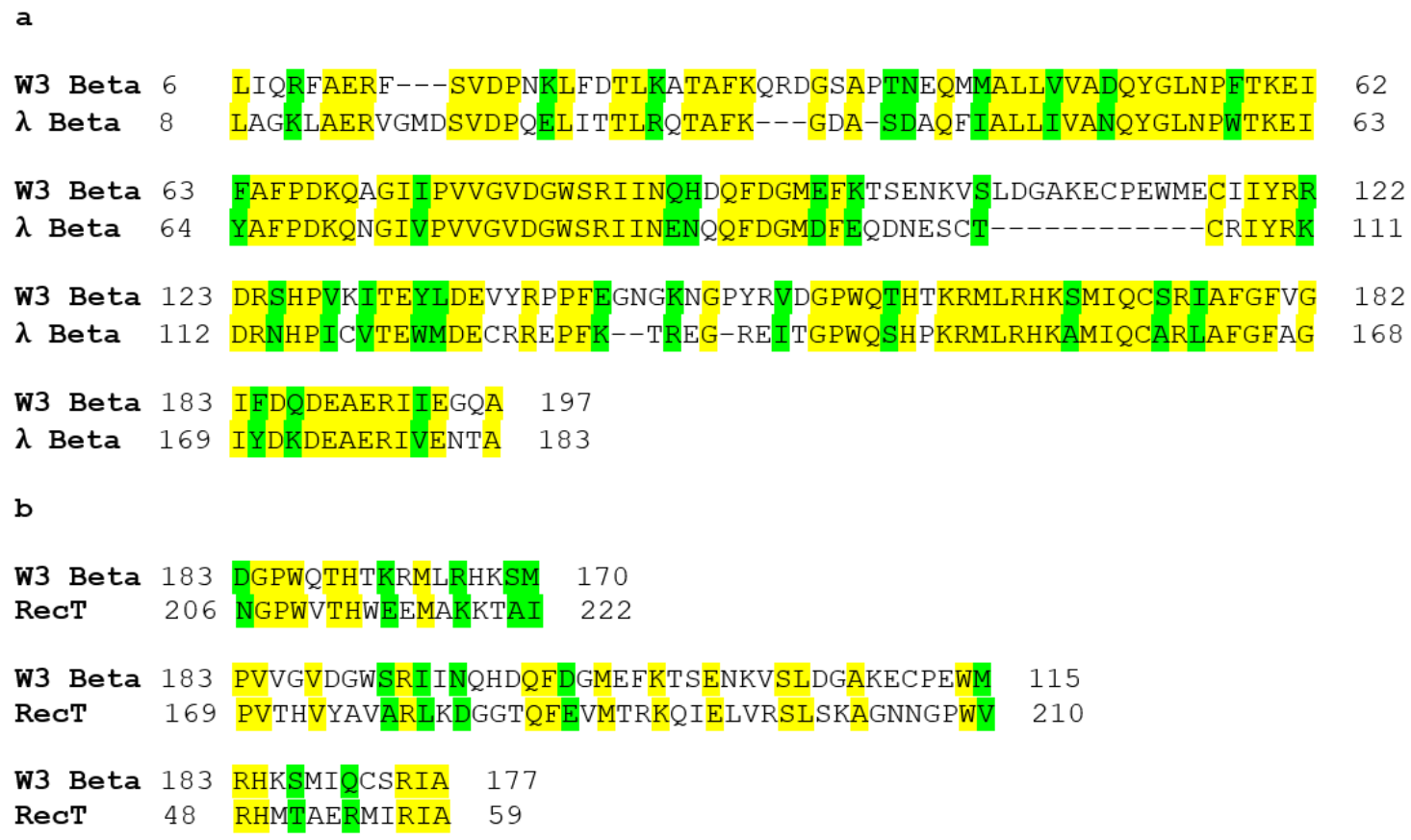

Figure 3. 3. Recombinase protein alignments.

The National Center Biotechnology Institute Blastp tool was used. Yellow highlighting indicates exact identity, whereas green indicates similarity. (a) W3 Beta versus $\lambda$ Red Beta. (b) W3 Beta versus RecT.

\subsubsection{Recombineering of lac Z in S. oneidensis}

We first evaluated oligo recombineering mediated by $\lambda$ Red Beta and RecT by targeting the $E$. coli lacZ gene integrated in a single copy on the $S$. oneidensis chromosome. Although in our plasmid transformation optimization we obtained higher efficiencies using late exponential phase cells, actively replicating chromosomes is fundamental when performing recombineering as these recombinases exploit replication forks (Thomason et al. 2016), thus we used cells at early exponential phase. Additionally, sorbitol was used as wash buffer since it gave the best results when using early exponential phase cells.

Previous studies in E. coli show that most single base pair mistmatches as well as insertions up to three nucleotides are corrected by the methyl-directed mismatch repair (MMR) system present in the cell (Modrich 1991; Costantino \& Court 2003; Sawitzke et al. 2011). On this basis, we used synthetic ssDNA oligos containing a 10 nucleotide (nt) mutation to disrupt lacZ (Fig. 3.4a), which evades the MMR and causes a mutant lacZ- phenotype that can be identified by white/blue screening(Shuman \& Silhavy 2003). 


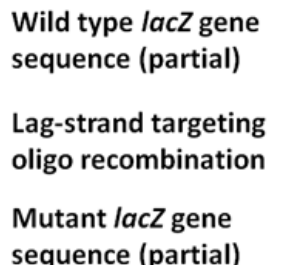

sequence (partial)
5'- CATTACGGTCAATCCGCCGTTTGTTCCCACGGAGAATCCGACGGGTTGTTACTCGCTCACATTTAATGTTGATGAAAGCTGGCTA- 3' 3'- GTAATGCCAGTTAGGCGGCAAACAAGGGTGCCTCTTAGGCTGCCCAACAATGAGCGAGTGTAAATTACAACTACTTTCGACCGAT- 5'

5'- CATTACGGTCAATCCGCCGTTTGTTCCCACGGAGAATCCG $\stackrel{\text { taataataa }}{\text { TTGTTACTCGCTCACATTTAATGTTGATGAAAGCTGGCTA- } 3}$ 3'- GTAATGCCAGTTAGGCGGCAAACAAGGGTGCCTCTTAGGCTGCCCAACAATGAGCGAGTGTAAATTACAACTACTTTCGACCGAT -

5'- CATTACGGTCAATCCGCCGTTTGTTCCCACGGAGAATCCGTAATAAGTAATTGTTACTCGCTCACATTTAATGTTGATGAAAGCTGGCTA- 3' 3'- GTAATGCCAGTTAGGCGGCAAACAAGGGTGCCTCTTAGGCATTATTCATTAACAATGAGCGAGTG TAAATTACAACTACTTTCGACCGAT- 5

Figure 3. 4. Disruption of chromosomal lacZ in S. oneidensis.

Sequence corresponding to the site of the mutation is shown in bold. A recombinogenic oligonucleotide was utilized to introduce ten consecutive base pair changes, which resulted in 10 bp mutations coupled with a frameshift, shown in red.

Unexpectedly, $\sim 1 \times 10^{4}$ recombinants in $10^{8}$ viable cells were obtained without the expression of a heterologous recombinase, suggesting that a low level of recombination occurs by a recombinase-independent process. However, neither $\lambda$ Red Beta or RecT were functional, since the number of recombinants obtained was marginally above the minus-recombinase control (Fig. 3.5a). Thus, we then tested the new recombinase W3 Beta, which was highly efficient for oligo recombineering (Fig. 3.5a), obtaining $\sim 5 \times 10^{6}$ recombinants in $10^{8}$ viable cells. No recombinants were observed in control experiments lacking the oligo in the system.

$\mathrm{Tu}$ and colleagues ( $\mathrm{Tu}$ et al. 2016) found that $\lambda$ Red recombineering of a plasmid with dsDNA in E. coli was improved $\sim 3$-fold when the electrocompetent cells were prepared on ice and shifted to room temperature for three minutes prior to electroporation. We sought to test recombineering with W3 Beta in S. oneidensis by preparing the electrocompetent cells at different temperatures. We found that room temperature prepared cells resulted in $\sim 6$-fold more recombinants when compared to preparing cells on ice, even if we allowed transient swelling of the cells for three minutes at room temperature (Fig. 3.5b). As in our plasmid transformations, we observed a decrease in cell viability when cells were prepared on ice. Similarly to E. coli (Sawitzke et al. 2011; Costantino \& Court 2003), most colonies were still lacZ $Z^{+}$, sectored blue and white, indicating the segregation of recombinant chromosomes in the original recombinant cell. Sectored colonies were streaked for isolated $l a c Z$ pure colonies, followed by PCR amplification of $l a c Z$ and sequencing of 27 colonies from these different temperature tests, which confirmed the mutations introduced (Fig. 3.5d).

It has been shown in E. coli that 40nt of homology upstream and downstream of the target site is sufficient to achieve high efficiency of oligo recombineering (Sawitzke et al. 2011). We tested the system using oligos containing different length of homology arms ranging from 40nt to $80 \mathrm{nt}$ to find the minimum optimal length in S. oneidensis. Although 80nt resulted in higher efficiency, generating up to $\sim 5 \%$ recombinants among treated cells, the difference was marginal, as we obtained $\sim 3 \%$ recombinants with the shortest 40 nt homology length (Fig. 3.5c). 
A clear bias for oligo annealing to the lagging strand versus the leading strand has been demonstrated in E. coli (Datta et al. 2008; Thomason et al. 2016; Ellis et al. 2001; Costantino \& Court 2003). We sought to evaluate this bias with W3 Beta in S. oneidensis. All the oligos tested corresponding in sequence to the leading strand and thus complement to the lagging strand (lagstrand oligo) generated higher numbers of recombinants than the oligos complementing the leading strand (lead-strand oligo) (Fig. 3.5c).

a
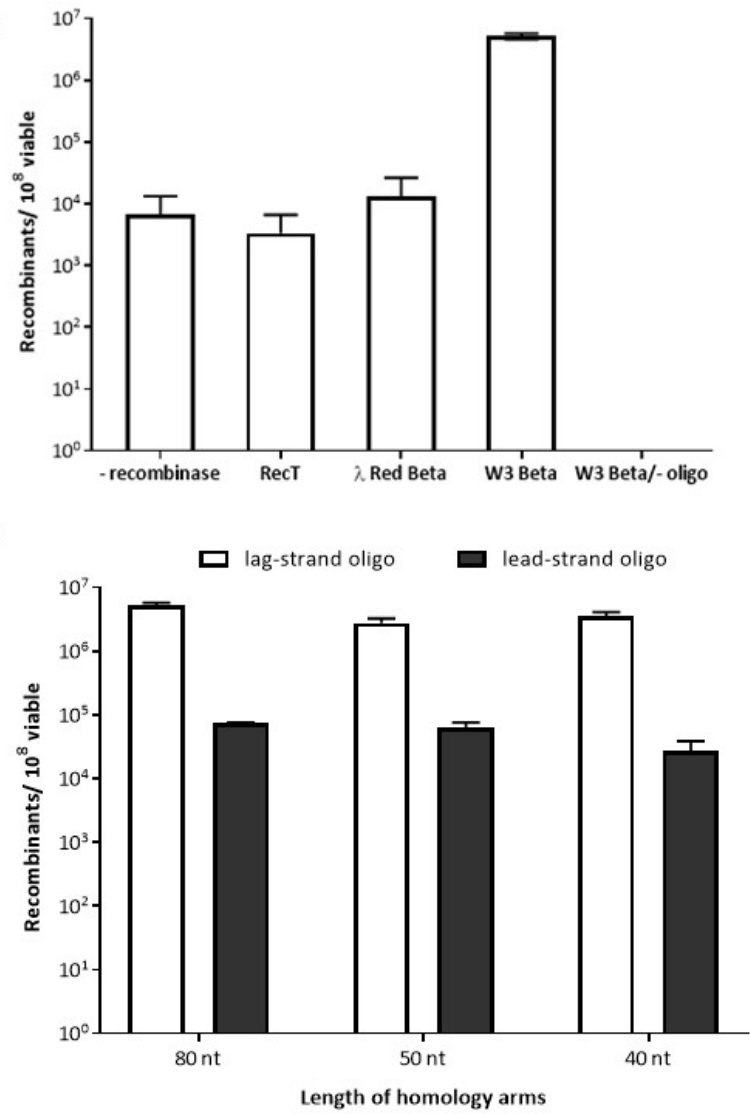

b
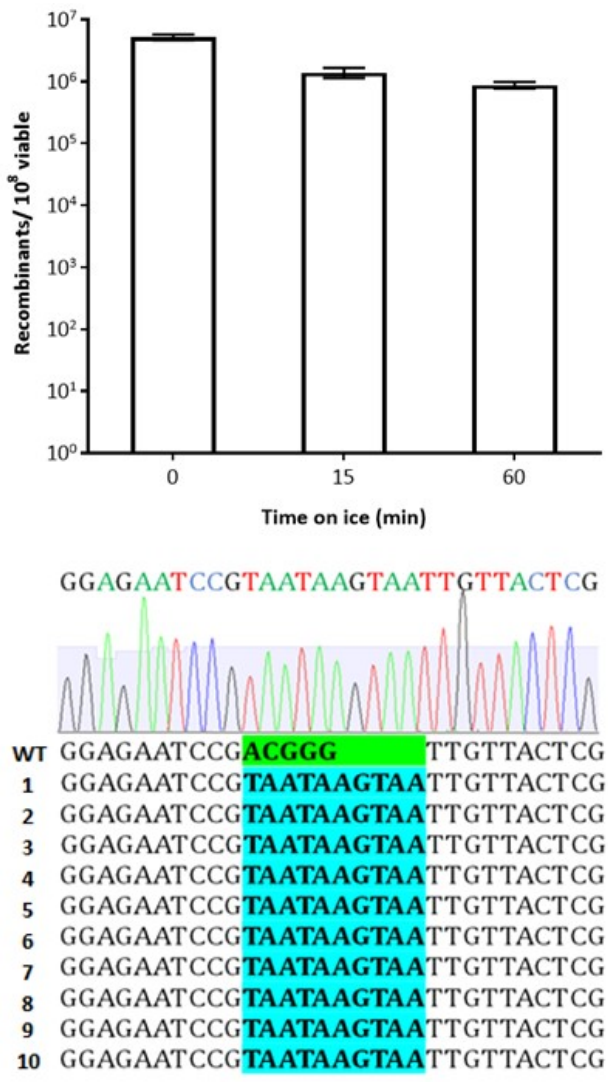

Figure 3. 5. Recombineering of $l a c Z$ in $S$. oneidensis using W3 Beta recombinase.

(a) Activity of different recombinases when expressed exogenously in $S$. oneidensis compared to recombinase-independent (pBTBX2 empty plasmid) recombination and a mock transformation control lacking mutagenic oligonucleotide (80nt homology arms, lag-strand oligo). (b) Effect of temperature when making electrocompetent cells on recombineering. Cells were prepared at room temperature or kept on ice (15 or 60 min before washing) followed by the conventional preparation on ice prior to electroporation (80nt homology arms, lag-strand oligo). (c) Homology arm length of the oligo and targeting strand effect on recombineering. The number of recombinants was calculated based on the lacZ- colonies on the X-gal plate. The error bars represent standard error from three independent experiments. (d) Sequence confirmation of recombinants identified by white/blue screening, which revealed the mutation of the DNA sequence ACGGG to TAATAAGTAA for all samples, as expected (10 mutants are shown here). 


\subsubsection{Recombineering of rpsL in S. oneidensis}

We further demonstrate oligo recombineering targeting a native gene in the chromosome of $S$. oneidensis, the rpsL gene. A recombinogenic oligo targeting the lagging strand (Fig 3.6a) with short homology arms of $40 \mathrm{nt}$ was used (Fig. 3.6b). Similar to Swingle and colleagues (Swingle, Bao, et al. 2010; Swingle, Markel, et al. 2010), the oligos were designed to introduce 4 bp changes containing the K43R (AAA->CGG) mutation, which confers streptomycin resistance (Strep ${ }^{\mathrm{R}}$ ), and a synonymous mutation in P42 (CCT->CCA) to evade MMR (Fig. 3.6b).

b

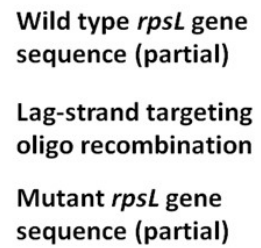

a
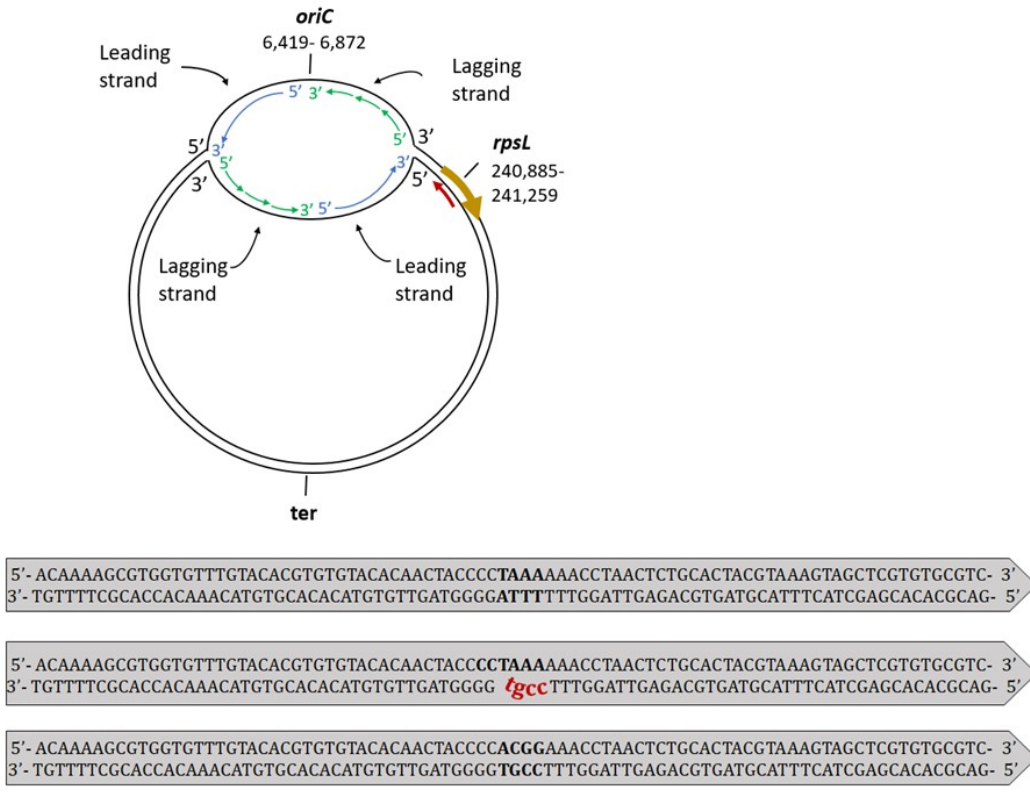

3'- TGTTTTCGCACCACAAACATGTGCACACATGTGTTGATGGGGTGCCTTTGGATTGAGACGTGATGCATTTCATCGAGCACACGCAG-

Figure 3. 6. Mutagenesis of chromosomal rpsL in $S$. oneidensis.

(a) Organization of the $S$. oneidensis MR-1 chromosome showing the location of $r p s L$ respective to the origin of replication ( $\mathrm{oriC}$ ). The relative position of oriC (determined using DoriC database) and terminus (estimated as the location of the origin plus half of the chromosome length) define two replichores through which DNA synthesis proceeds. As the oligos mimic Okazaki fragments at the replication fork, the orientation of the target gene in respect to the oriC needs to be known beforehand for an optimal recombineering efficiency. Shown in red is the 5' $\rightarrow$ 3' alignment of the recombinogenic oligo, complement to the lagging strand template, to target $r p s L$. (b) Sequence corresponding to the site of the mutation is shown in bold. A recombinogenic ooligo targeting the lagging strand was utilized to introduce four consecutive base pair changes, which resulted in the K43R mutation and a synonymous mutation in P42, shown in red.

Because the frequency of recombination was determined by the number of colonies that grew on streptomycin-containing LB agar, a longer outgrowth was necessary to allow segregation of the recombinant allele before selection (Fig. 3.7a). Again, a difference in efficiency was observed based on the target strandedness, specifically $\sim 3 \times 10^{6}$ and $\sim 3 \times 10^{4}$ recombinants were 
obtained when targeting the lagging and leading strand, respectively (Fig. 3.7b). The percentage of recombinants among viable cells was lower than when editing lac $Z, \sim 1 \%$ targeting the lagging strand, due to the increased recovery step to obtain pure rpsLK43R genotypes (Pines et al. 2015). Without the addition of the recombinase in the cells, $\sim 3 \times 10^{4}$ recombinants were obtained by targeting the lagging strand, in agreement with our lacZ editing experiments. Ten mutants each from the + and - recombinase experiments were screened by colony PCR with a reverse primer specific to the mutation introduced, which revealed that all recombinants contained the correct 4 bp change (Fig. 3.8a). Sequencing further confirmed the success of the experiment (Fig. 3.8b).

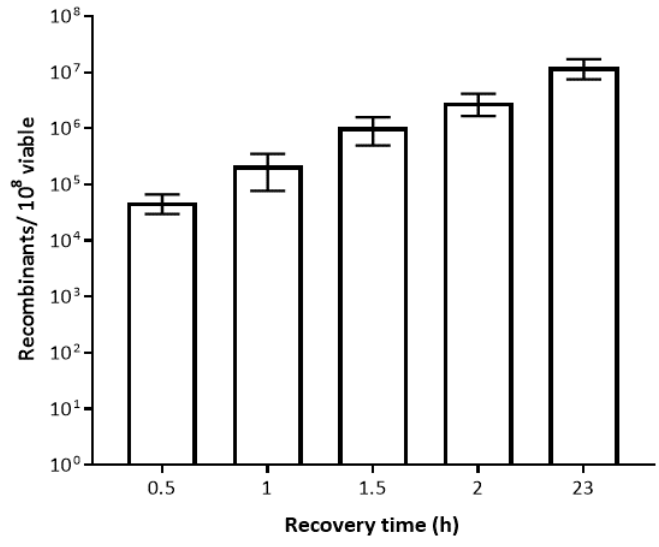

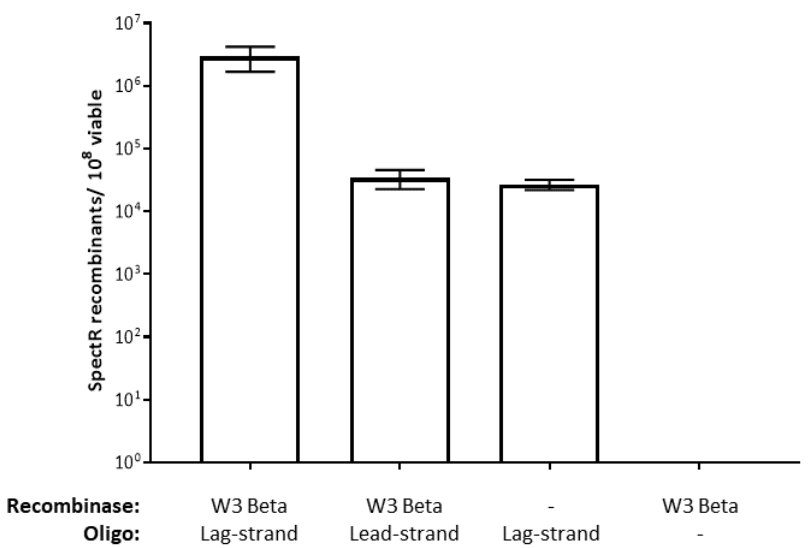

Figure 3. 7. Recombineering of $r p s L$ in $S$. oneidensis using W3 Beta recombinase.

(a) Effect of recovery time on recombineering of the rpsLK43R oligo (40nt homology arms, lagstrand oligo). (b) Targeting strand effect on recombineering compared to recombinase-independent and mock transformation lacking mutagenic oligonucleotide controls (40nt homology arms oligo, $2 \mathrm{~h}$ recovery). The number of recombinants was calculated based on the Spect ${ }^{\mathrm{R}}$ colonies. The error bars represent standard error from three independent experiments. 
a

$\begin{array}{lllllllllll}1 & 2 & 3 & 4 & 5 & 6 & 7 & 8 & 9 & 10 & \emptyset\end{array}$

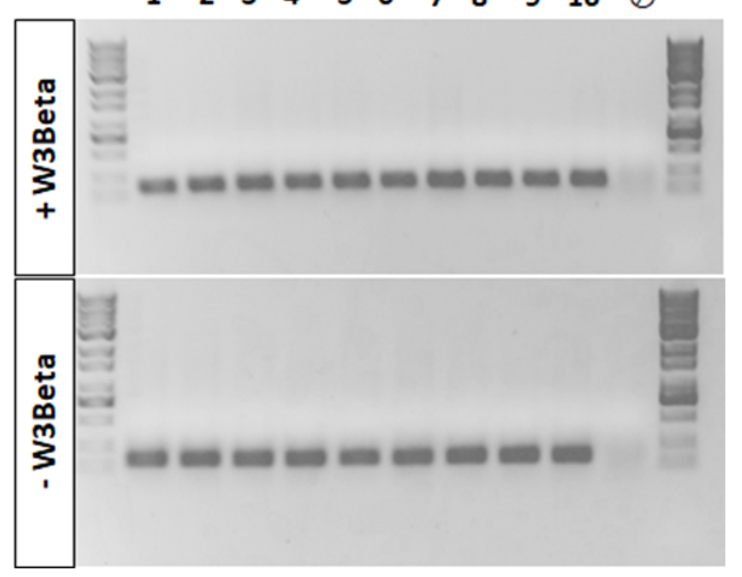

b

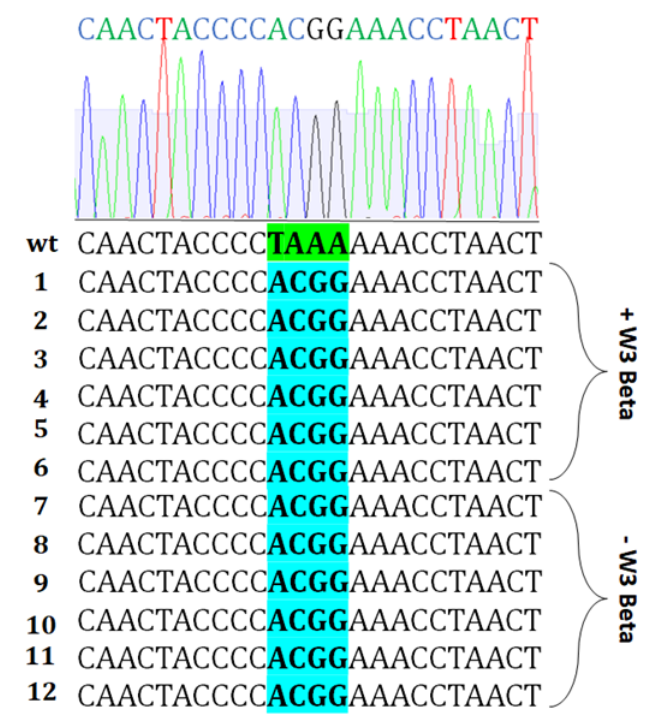

Figure 3. 8. S. oneidensis rps $L K 43 R$ recombinants verification.

Mutants were identified as streptomycin resistant (StepR) colonies. (a) Ten mutants from each experiment, the $+\mathrm{W} 3$ Beta and - W3 Beta (pBTBX2 empty plasmid control), were screened by colony PCR with a reverse primer specific to the DNA change introduced, validating the correct mutation in all colonies. $\oslash$ indicates a PCR negative control of wild-type rpsL. The gel was cropped to show the relevant information and no high-contrast of the image was used. (b) Sequence confirmation of 6 recombinants identified by StrepR from each experiment, which further revealed the mutation of the DNA sequence TAAA to ACGG for all samples, as expected.

\subsubsection{Recombineering of $r p s L$ in $S$. amazonensis}

In order to asses our system in $S$. amazonensis, we employed the same methodology and targeted the lagging strand of the rpsL (Figure 3.9). Using an oligo with 40 nt homology arms (Table 3.3), we obtained $\sim 10^{5}$ spectinomycin resistant (SpectR) CFUs among total viable cells (Figure 3.10a), a 100-fold lower frequency compared to MR-1 (Corts et al. 2019). However, colony PCR and sequencing confirmed the engineered mutation TAAG-> ACGG (Figure 3.10b, c).

We observed that $S$. amazonensis is slightly resistant to $100 \mu \mathrm{g} / \mathrm{ml}$ spectinomycin; about $10^{3} \mathrm{cfu} / \mathrm{mL}$ were obtained in the controls with either no oligo or no recombinase present (Figure 3.10a). Increasing the concentration of spectinomycin did not eliminate this background.

Although, ssDNA recombineering may require some optimization in S. amazonensis, this efficiency is adequate to allow construction of single mutants and shows promise for the application in other Shewanella strains when electroporation is possible 
b)

Wild type $r p s L$ gene sequence (partial)

Lag-strand targeting oligo recombination

Mutant rpsL gene sequence (partial) a)

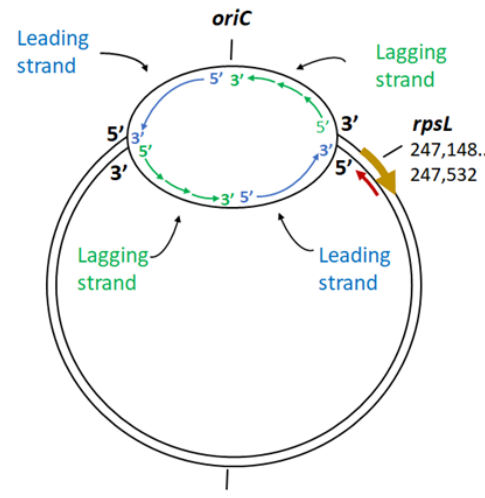

ter

5' - ACAAAAGCGTGGTGTTTGTACACGTGTGTACACAACTACCCCTAAAAAACCTAACTCTGCACTACGTAAAGTAGCTCGTGTGCGTC- 3' 3'- TGTTTTCGCACCACAAACATGTGCACACATGTGTTGATGGGGATTTTTTGGATTGAGACGTGATGCATTTCATCGAGCACACGCAG- 5'

5'- ACAAAAGCGTGGTGTTTGTACACGTGTGTACACAACTACCCCTAAAAAACCTAACTCTGCACTACGTAAAGTAGCTCGTGTGCGTC3'- TGTTTTCGCACCACAAACATGTGCACACATGTGTTGATGGGG 'gcc TTTGGATTGAGACGTGATGCATTTCATCGAGCACACGCAG-

5'- ACAAAAGCGTGGTGTTTGTACACGTGTGTACACAACTACCCCACGGAAACCTAACTCTGCACTACGTAAAGTAGCTCGTGTGCGTC3'- TGTTTTCGCACCACAAACATGTGCACACATGTGTTGATGGGGTGCCTTTGGATTGAGACGTGATGCATTTCATCGAGCACACGCAG- 5',

Figure 3. 9. Mutagenesis of chromosomal rpsL in $S$. amazonensis.

(a) Organization of the $S$. amazonensis SB2B chromosome showing the location of $r p s L$ respective to the origin of replication ( $\mathrm{oriC}$ ). The relative position of oriC and terminus (estimated as the location of the origin plus half of the chromosome length) define two replichores through which DNA synthesis proceeds. As the oligos mimic Okazaki fragments at the replication fork, the orientation of the target gene in respect to the oriC needs to be known beforehand for an optimal recombineering efficiency. Shown in red is the $5^{\prime} \rightarrow 3^{\prime}$ alignment of the recombinogenic oligo, complement to the lagging strand template, to target rpsL. (b) Sequence corresponding to the site of the mutation is shown in bold. A recombinogenic oligo targeting the lagging strand was utilized to introduce four consecutive base pair changes, which resulted in the K43R mutation and a synonymous mutation in $\mathrm{P} 42$, shown in red. 
b)

$\begin{array}{llllllllllll}1 & 2 & 3 & 4 & 5 & 6 & 7 & 8 & 9 & 10 & \emptyset\end{array}$
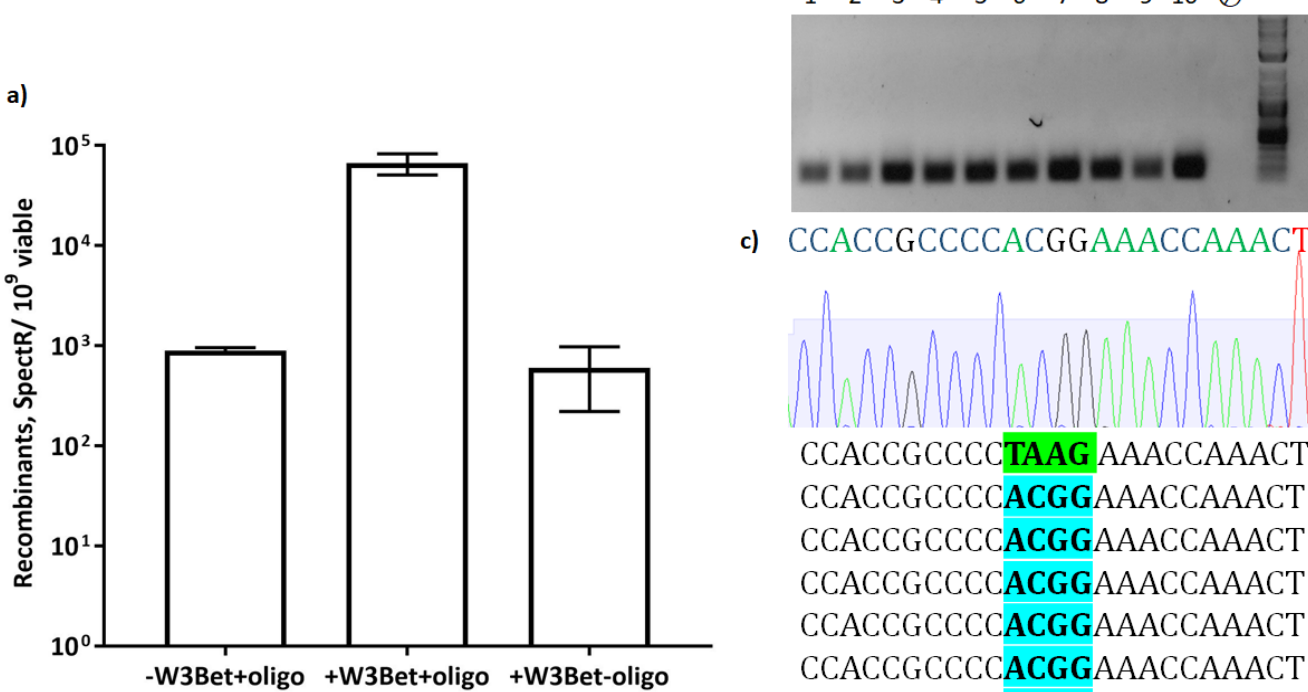

c) CCACCGCCCCACGGAAACCAAACT

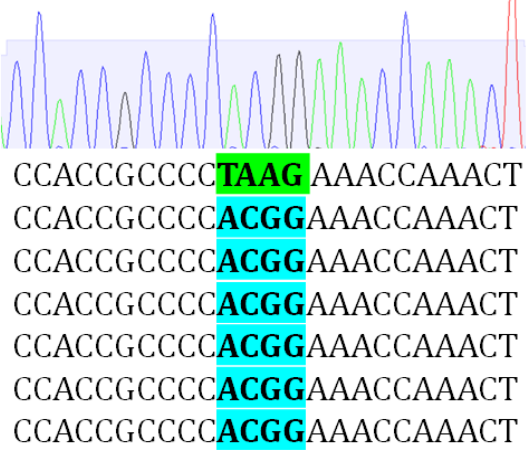

Figure 3. 10. Recombineering in S. amazonensis SB2B.

(a) Total SpectR colonies from recombineering with W3 Beta and SAMArpsLK43R ssDNA oligo (40nt homology arms, lag-strand oligo). A minus-recombinase and a minus-oligo control are included. Cells were grown to early-exponential phase with arabinose to induce W3 Beta, then cells were made electrocompetent and ssDNA oligo was transformed. Cells were recovered for 2 hours prior to plating on $\mathrm{LB}+\mathrm{km}+$ Spect. The error bars represent standard error from three independent experiments. (b) 10 mutants were screened by colony PCR with a reverse primer specific to the DNA change introduced, validating the correct mutation in all colonies. $\oslash$ indicates a PCR negative control of wild-type rpsL. The gel was cropped to show the relevant information and no high-contrast of the image was used. (c) Sequence confirmation of recombinants identified as SpectR, which revealed the mutation of the DNA sequence TAAG to ACGG for all samples, as expected (6 mutants are shown here).

\subsubsection{W3 Beta is a broad host recombinase}

Surprisingly, W3 Beta was also functional in E. coli at an efficiency comparable to $\lambda$ Red Beta. In fact, we observed that preparing electrocompetent cells and performing electroporation at room temperature also increased the number of recombinants $\sim 5$-fold when using $\lambda$ Red Beta and W3 Beta in E. coli (Figure 3.11a), in contrast to the dsDNA plasmid recombineering previous findings ( $\mathrm{Tu}$ et al. 2016). Accomplishment of the DNA change introduced by W3 Beta was confirmed by colony PCR of 14 mutants with a reverse primer specific to the mutation and sequencing of 10 colonies (Figure 3.11b,c). 

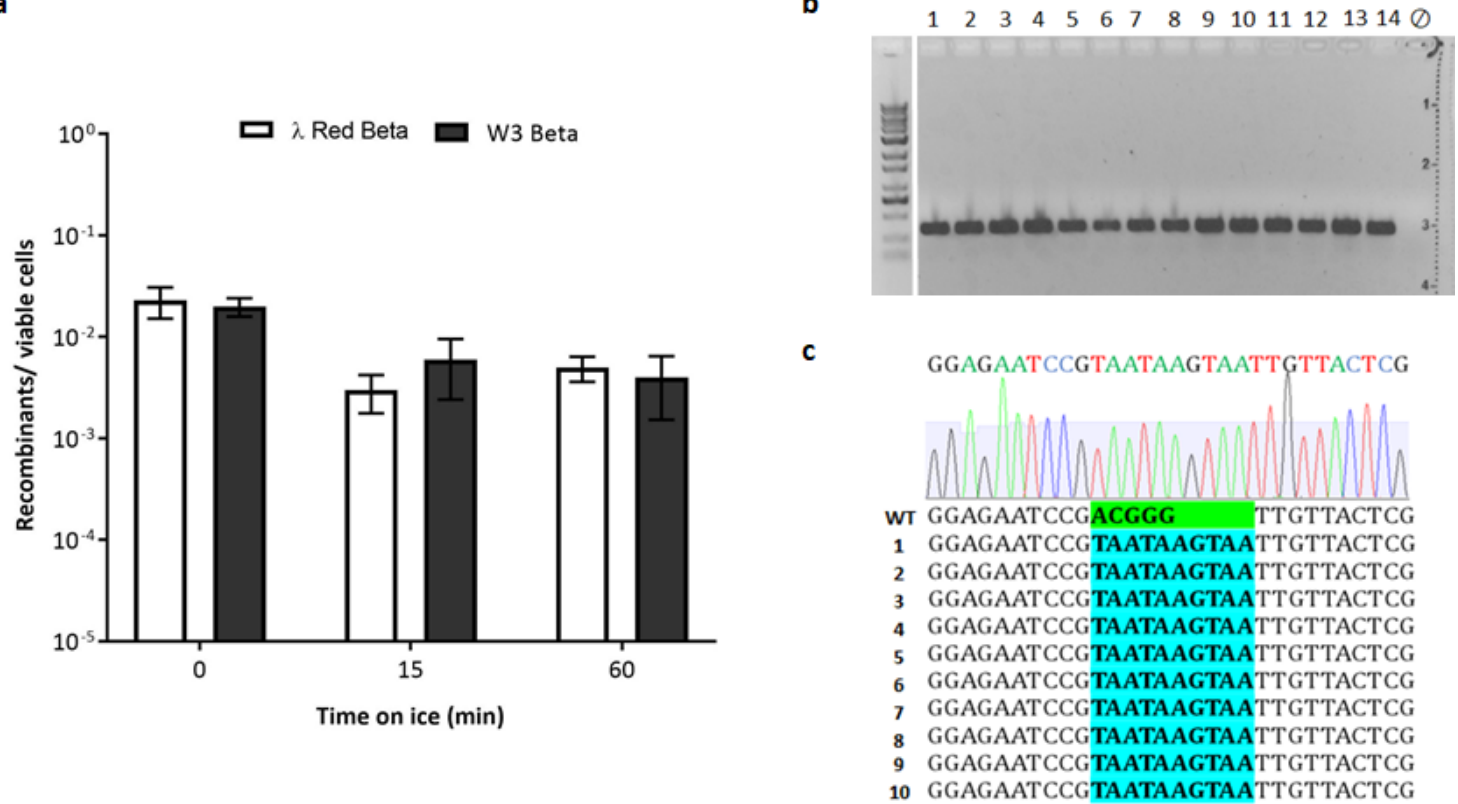

Figure 3. 11. ssDNA recombineering in $E$. coli MG1655 by preparing electrocompetent cells at different temperatures.

(a) Comparison of $\lambda$ Red Beta and W3 Beta activity in ssDNA recombineering using a lag-strand targeting oligo with 80nt homology arms to disrupt lacZ, as shown in Fig. 3.4. The number of recombinants was calculated by scoring blue colonies containing white sectors on the $\mathrm{LB}+\mathrm{Km}+$ $\mathrm{X}$-gal agar plates. The error bars represent standard error from three independent experiments. (b) Sectored colonies were streaked for isolated lacZ pure colonies and screened by colony PCR with a reverse primer specific to the DNA change introduced, validating the correct mutation in all colonies. $\oslash$ indicates a PCR negative control of wild-type lacZ. The gel was cropped to show the relevant information and no high-contrast of the image was used. The molecular size marker was run on the same gel as the samples shown. The white line indicates the gel contained other samples not relevant. (c) Sequence confirmation of 10 recombinants identified by white/blue screening, which revealed the mutation of the DNA sequence ACGGG to TAATAAGTAA for all samples, as expected.

\subsection{Discussion}

Homologous recombination using phage functions, known as recombineering, which has been coupled with electrotransformation in E. coli (Sharan et al. 2009) and a few other bacteria (van Kessel \& Hatfull 2007; Yin et al. 2015; Yang et al. 2015; Swingle, Bao, et al. 2010; Oh \& Van Pijkeren 2014; Aparicio et al. 2018; Penewit et al. 2018; Lee et al. 2017), is a powerful tool for in vivo genome editing. In E. coli, recombineering has enabled targeted multiplexed editing strategies such as MAGE (Multiplex Automated Genome Engineering) (Wang et al. 2009), TRMR (Trackable Multiplex Recombineering) (Warner et al. 2010) and CREATE (CRISPR EnAbled Trackable genome Engineering) (Garst et al. 2016), all which take advantage of massive DNA 
delivery by electroporation, highlighting the importance of this transformation method when performing recombineering experiments.

In contrast to traditional genetic engineering strategies, recombineering allows researchers to rapidly and precisely introduce a variety of changes using linear DNA fragments. The use of ssDNA for recombineering, which was first described in E. coli over ten years ago (Ellis et al. 2001), is independent of a selective marker, does not leave any extraneous genetic scars and is mechanistically simpler than using dsDNA, requiring only the expression of the recombinase alone. However, the well-studied $\lambda$ Red Beta and RecT recombinases were not functional in S. oneidensis. The number of recombinants obtained when expressing $\lambda$ Red Beta or RecT was marginally above that of the recombinase-independent control. As shown in other bacteria (Swingle, Markel, et al. 2010), S. oneidensis is able to recombine ssDNA in a recombinase-independent way at a frequency of $\sim 10^{-4}$ recombinants/viable cells. According to Li and colleagues (Li et al. 2013), host factors such as replicative DNA polymerases and DNA ligase can play a role in completing recombination of the ssDNA at the replication fork (Li et al. 2013), which could explain why $\lambda$ Red Beta and RecT are specific to E. coli and did not promote recombination in $S$. oneidensis.

In this study, we identified a $\lambda$ Red Beta recombinase homolog in Shewanella sp. W3-181, termed W3 Beta, and demonstrated its functionality for ssDNA recombineering in S. oneidensis. We obtained a recombination efficiency of $\sim 5 \%$, similar to that of studies in E. coli (Ellis et al. 2001) and higher than previous $\lambda$ Red Beta and RecT homologous systems found in other bacteria (van Kessel \& Hatfull 2007; Yin et al. 2015; Yang et al. 2015; Swingle, Bao, et al. 2010; Oh \& Van Pijkeren 2014; Aparicio et al. 2018; Penewit et al. 2018; Lee et al. 2017). In addition, W3 Beta was also functional in E. coli and performed at similar efficiencies to $\lambda$ Red Beta. The observation that W3 Beta outperforms other systems and that it is also functional in phylogenetically distant bacteria highlights the efficient recombineering activity of this recombinase.

Like other phage recombinases, W3 Beta appears to act at the replication fork since it displays a strand bias, as do similar systems (Lee et al. 2017; Datta et al. 2008). We found that recombineering with W3 Beta results in higher efficiency when electrocompetent cells are prepared at room temperature instead of ice-cold temperature in S. oneidensis and E. coli, in agreement with our results using $\lambda$ Red Beta in E. coli.

The recombineering system described here is the first effective and simple strategy for targeted and markerless genome-editing in S. oneidensis, yielding a high efficiency of recombinant formation that permits screening for desired mutations in the absence of selection. Our system was developed without strain engineering, however rational removal of single-strand exonucleases could potentially enhance efficiency as shown in $V$. cholera (Dalia et al. 2017) and E. coli (Mosberg 
et al. 2012; Costantino \& Court 2003; Li et al. 2013). In chapter IV, we show that coupling the recombineering system developed here with the cutting edge CRISPR/Cas9-technology (Ronda et al. 2016; Yan et al. 2017), enables facile isolation of recombinants. In its current state, however, this system should facilitate genome editing projects that require precise modifications of small to medium-throughput scale. We postulate that this study will pave the way to further strain engineering of $S$. oneidensis.

\subsection{Materials and Methods}

\subsubsection{Bacterial Strains, plasmids and culture conditions}

All strains and plasmids used in this study are listed in Table 3.1. Shewanella was routinely grown in LB liquid medium or on LB agar at $30^{\circ} \mathrm{C}$. When needed, culture media was supplemented kanamycin $(\mathrm{Km}, 50 \mu \mathrm{g} / \mathrm{mL}$ ) or spectinomycin (Spect, $50 \mu \mathrm{g} / \mathrm{mL}$ ). Plasmid pBTBX2 from UQ950 and methylation-minus E. coli GM2163.

Strain JG2150 was constructed by an in-frame insertion of lacZ from E. coli MG1655 under the native promoter of $m t r C$ at the $g \operatorname{lmS}$ site in S. oneidesis MR-1. In brief, $1 \mathrm{~kb}$ regions upstream and downstream of $g \operatorname{lm} S$ were amplified and ligated into a suicide vector, pSMV3, which was transferred into MR-1 and screened for double recombination events, as previously described (Coursolle \& Gralnick 2010). 
Table 3. 1. Strains and plasmids used in this work.

\begin{tabular}{|c|c|c|}
\hline Strain or plasmid & Description & Reference or source \\
\hline \multicolumn{3}{|l|}{ Strain } \\
\hline $\mathrm{JG} 274$ & S. oneidensis MR-1, wild type & Gralnick Lab \\
\hline JG239 & Shewanella sp. W3-18-1 & Gralnick Lab \\
\hline JG98 & S. amazonensis SB2B & Gralnick Lab \\
\hline JG2150 & S. oneidensis, lacZ & Gralnick Lab \\
\hline JG3653 & S. oneidensis, pBTBX2 & This study \\
\hline JG4239 & S. oneidensis, pBTBX2 & This study \\
\hline JG4123 & S. oneidensis, pX2SW3Beta & This study \\
\hline JG4240 & S. amazonensis, pX2SW3Beta & This study \\
\hline JG3909 & S. oneidensis, $\mathrm{pX} 2 \lambda \mathrm{Beta}$ & This study \\
\hline JG3916 & S. oneidensis, pX2RecT & This study \\
\hline JG4140 & S. oneidensis, lacZ, pBTBX2 & This study \\
\hline JG4127 & S. oneidensis, lacZ, pX2SW3Beta & This study \\
\hline JG3940 & 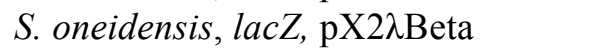 & This study \\
\hline JG3942 & S. oneidensis, lacZ, pX2RecT & This study \\
\hline UQ950 & E. coli DH5 $\alpha \lambda$ (pir) host for cloning & $\begin{array}{l}\text { (Saltikov \& Newman } \\
\text { 2003) }\end{array}$ \\
\hline GM2163 & E. coli methylation-minus, $\mathrm{CmR}$ & CGSC\#: 6581 \\
\hline GM1674 & E. coli methylation-minus & CGSC\#7971 \\
\hline MG1655 & E. coli F- lambda- $i l v G-r f b-50 r p h-1$ & Gralnick Lab \\
\hline JG4130 & E. coli, pX2SW3Beta & This study \\
\hline JG4128 & E. coli, $\mathrm{pX} 2 \lambda \mathrm{Beta}$ & This study \\
\hline \multicolumn{3}{|l|}{ Plasmid } \\
\hline pBTBX2 & pBBR1 ori, KmR, pBAD & (Prior et al. 2010) \\
\hline $\mathrm{pX} 2 \mathrm{Cas} 9$ & pBBR 1 ori, $\mathrm{KmR}$, pBAD, Cas9 & Gill lab (Garst et al. 2016) \\
\hline pSIM5 & pSC101 ori, cmR, pL, $\lambda$ Red & Court lab \\
\hline pBAD-ETg & pSC101 ori, pBAD, recET, $\lambda$ Gam & $\begin{array}{l}\text { Stewart Lab (Fu et al. } \\
\text { 2012) }\end{array}$ \\
\hline pX2SW3Beta & pBBR1 ori, KmR, pBAD, W3Beta & This study \\
\hline $\mathrm{pX} 2 \lambda$ Beta & pBBR1 ori, $\mathrm{KmR}$, pBAD, $\lambda$ Red Beta & This study \\
\hline $\mathrm{pX} 2 \operatorname{RecT}$ & pBBR1 ori, KmR, pBAD, RecT & This study \\
\hline
\end{tabular}




\subsubsection{DNA manipulations}

All primers used in this study are listed in Table 3.2. Plasmid DNAs were isolated using Invitrogen Plasmid mini Kit.

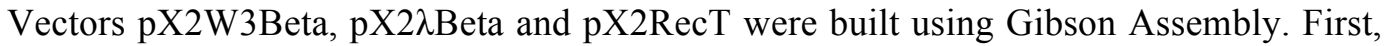
plasmid pX2Cas9 was linearized by PCR amplification. The recombinase encoding genes were PCR amplified with overhang sequences for assembly; $s w_{-} 1153$ was PCR amplified from a Shewanella $\mathrm{sp}$. W3-18-1 colony, $\lambda$ beta from plasmid pSIM5 and recT from pBAD-ETg.

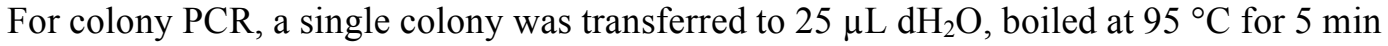
and frozen at $-80{ }^{\circ} \mathrm{C}$ for $5 \mathrm{~min}$. The cell debris was removed by centrifugation for $1 \mathrm{~min}$ and $2 \mu \mathrm{L}$ of the supernatant were used as a source of template in a $20 \mu \mathrm{L}$ reaction. GoTaq $2 \mathrm{X}$ MM (Promega) was used and supplier instructions were followed. After PCR, $10 \mu \mathrm{L}$ aliquots were analyzed by agarose gel electrophoresis.

Table 3. 2. Primers used in this study.

\begin{tabular}{|c|c|c|}
\hline \multicolumn{3}{|c|}{ Name $\quad$ Sequence $\left(5^{\prime}->3^{\prime}\right)^{a}$} \\
\hline \multicolumn{3}{|c|}{ Primers for construction of recombineering plasmids } \\
\hline ADC102 & $\begin{array}{l}\text { GGGTATATCTCCTTCTTAAAGTTC } \\
\text { GTATC }\end{array}$ & $\begin{array}{l}\text { Rev primer linearize } \mathrm{pX} 2 \mathrm{Cas} 9 \text { for } \\
\text { cloning the recombine ORF in place of } \\
\text { cas } 9\end{array}$ \\
\hline ADC103 & TCTAGAGAATTCGTCAACGA & $\begin{array}{l}\text { For primer linearize } \mathrm{pX} 2 \mathrm{Cas} 9 \text { for } \\
\text { cloning the recombinase ORF in place } \\
\text { of cas } 9\end{array}$ \\
\hline $\mathrm{ADC} 116$ & $\begin{array}{l}\text { cgaactttaagaaggagatatacccATGAGTAC } \\
\text { TGCACTCGCAACG }\end{array}$ & $\begin{array}{l}\text { For primer to amplify } \lambda \text {-beta for } \\
\text { cloning in place of } \operatorname{cas} 9\end{array}$ \\
\hline $\mathrm{ADC} 120$ & $\begin{array}{l}\text { cgttgacgaattctctagaTCATGCTGCCACC } \\
\text { TTCTGCT }\end{array}$ & $\begin{array}{l}\text { Rev primer to amplify } \lambda \text {-beta for } \\
\text { cloning in place of cas } 9\end{array}$ \\
\hline ADC119 & $\begin{array}{l}\text { cgaactttaagaaggagatatacccATGACTAA } \\
\text { GCAACCACCAATC }\end{array}$ & $\begin{array}{l}\text { For primer to amplify racT for cloning } \\
\text { in place of cas } 9\end{array}$ \\
\hline ADC118 & $\begin{array}{l}\text { cgttgacgaattctctagaTTATTCCTCTGAA } \\
\text { TTATCGATTACACTG }\end{array}$ & $\begin{array}{l}\text { Rev primer to amplify racT for cloning } \\
\text { in place of cas } 9\end{array}$ \\
\hline $\mathrm{ADC} 133$ & $\begin{array}{l}\text { cgaactttaagaaggagatatacccATGGAAAA } \\
\text { ACCAAAGCTAATCCAACG }\end{array}$ & $\begin{array}{l}\text { For primer to amplify sw_ } 1153 \text { for } \\
\text { cloning in place of cas } 9\end{array}$ \\
\hline ADC132 & $\begin{array}{l}\text { cgttgacgaattctctagaCTAAGAAGCTAA } \\
\text { AGGCTGTGTGAGCG }\end{array}$ & $\begin{array}{l}\text { Rev primer to amplify } \mathrm{s} w \_1153 \text { for } \\
\text { cloning in place of cas } 9\end{array}$ \\
\hline \multicolumn{3}{|c|}{ Primers for plasmid sequence verification } \\
\hline ADC126 & ATACCAGGATCTTGCCATCC & For primer to sequence $\operatorname{araC}$ \\
\hline $\mathrm{ADC} 121$ & TATGGAACTGCCTCGGTGAG & For primer to sequence $\operatorname{araC}$ \\
\hline ADC122 & GACGAAAGTAAACCCACTGG & For primer to sequence $\operatorname{araC}$ and $\mathrm{P}_{\mathrm{BAD}}$ \\
\hline $\mathrm{ADC} 31$ & ATTAGCGGATCCTACCTGACG & $\begin{array}{l}\text { For primer to sequence the } \\
\text { recombinase ORF }\end{array}$ \\
\hline $\mathrm{ADC} 33$ & CGCATCATCAATGAAAACCAGCAG & For primer to sequence $\lambda$-beta \\
\hline
\end{tabular}




\begin{tabular}{|c|c|c|}
\hline ADC194 & ATCCATGATCCAGTGTTCCCGC & For primer to sequence $\mathrm{s} w \_1153$ \\
\hline ADC105 & AATCCCTGTGGTCAAGCTCG & $\begin{array}{l}\text { Rev primer to sequence verify soxR } \\
\text { terminator and the recombinase ORF }\end{array}$ \\
\hline $\mathrm{ADC} 110$ & & For primer to sequence verify $r a c T$ \\
\hline \multicolumn{3}{|c|}{ Primers for plasmid analysis } \\
\hline $\mathrm{ADC} 44$ & GTTTCACTTGATGCTCGATGAG & $\begin{array}{l}\text { For primer to check for the presence of } \\
\text { plasmid pBTBX2 in the cells }\end{array}$ \\
\hline ADC105 & AATCCCTGTGGTCAAGCTCG & $\begin{array}{l}\text { Rev primer to check for the presence } \\
\text { of plasmid pBTBX } 2 \text { in the cells }\end{array}$ \\
\hline \multicolumn{3}{|c|}{ Primers for screening recombinants } \\
\hline \multicolumn{3}{|c|}{ S. oneidensis } \\
\hline ADC51 & TGGCGTAATAGCGAAGAGG & $\begin{array}{l}\text { For primer to PCR amplify lacZ and for } \\
\text { sequencing }\end{array}$ \\
\hline ADC52 & CCTGATCTTCCAGATAACTGCC & $\begin{array}{l}\text { Rev primer to PCR amplify lacZ for } \\
\text { sequencing }\end{array}$ \\
\hline ADC207 & $\begin{array}{l}\text { GTGAGCGAGTAACAATTACTTAT } \\
\text { TA }\end{array}$ & $\begin{array}{l}\text { Rev primer specific to the mutated lacZ } \\
\text { for PCR amplification/screening }\end{array}$ \\
\hline ADC206 & ATGGCAACTGTAAACCAG & $\begin{array}{l}\text { For primer to PCR amplify rps } L \text { and for } \\
\text { sequencing }\end{array}$ \\
\hline ADC205 & TTAAGACTTAGGACGCTTAGC & $\begin{array}{l}\text { Rev primer to PCR amplify rpsL for } \\
\text { sequencing }\end{array}$ \\
\hline ADC208 & CAGAGTTAGGTTTCCGTGG & $\begin{array}{l}\text { Rev primer specific to the mutated } r p s L \\
\text { for PCR amplification/screening }\end{array}$ \\
\hline \multicolumn{3}{|c|}{ S. amazonensis } \\
\hline ADC229 & AAATCAGTTGGTACGTAAGCC & $\begin{array}{l}\text { For primer to PCR amplify } r p s L \text { and for } \\
\text { sequencing }\end{array}$ \\
\hline $\mathrm{ADC} 230$ & TTAAGACTTAGGACGCTTGGC & $\begin{array}{l}\text { Rev primer to PCR amplify } r p s L \text { for } \\
\text { sequencing }\end{array}$ \\
\hline $\mathrm{ADC} 231$ & GTGCAGAGTTTGGTTTCCGT & $\begin{array}{l}\text { Rev primer specific to the mutated } r p s L \\
\text { for PCR amplification }\end{array}$ \\
\hline
\end{tabular}

${ }^{a}$ Lower case designates Gibson assembly overhangs.

\subsection{3. ssDNA oligo recombineering in $S$. oneidensis and $S$. amazonensis}

All oligos described in Table S2, were ordered from IDT as 4 nmole ultramer dried DNA and suspended in $\mathrm{dH}_{2} \mathrm{O}$ to a concentration of $0.5 \mu \mathrm{g}$. 
Table 3. 3. Oligonucleotides used for chromosomal mutations.

\begin{tabular}{|c|c|}
\hline Name & Sequence (5'-> 3') \\
\hline 80nt-lag-lacZ & $\begin{array}{l}\text { 5'acggttacgatgcgeccatctacaccaacgtgacctatcccattacggtcaatccgccgtttgt } \\
\text { tcccacggagaatccgTAATAAGTAAttgttactcgctcacatttaatgttgatgaaagc } \\
\text { tggctacaggaaggccagacgcgaattattttgatggegttaact }\end{array}$ \\
\hline 80nt- lead-lacZ & $\begin{array}{l}\text { 5'agttaacgccatcaaaaataattcgcgtctggcettcctgtagccagctttcatcaacattaaat } \\
\text { gtgagcgagtaacaaTTACTTATTAcggattctccgtgggaacaaacggeggattga } \\
\text { ccgtaatgggataggtcacgttggtgtagatgggcgcatcgtaaccgt }\end{array}$ \\
\hline 50nt- lag- lacZ & $\begin{array}{l}\text { 5'tgacctatcccattacggtcaatccgccgtttgttccacggagaatccgTAATAAGT } \\
\text { AAttgttactcgctcacatttaatgttgatgaaagetggctacaggaaggec }\end{array}$ \\
\hline 50nt- lead-lacZ & $\begin{array}{l}\text { 5'ggcettcctgtagccagctttcatcaacattaaatgtgagcgagtaacaaTTACTTATT } \\
\text { Acggattctccgtgggaacaaacggeggattgaccgtaatgggataggtca }\end{array}$ \\
\hline 40nt- lag-lacZ & $\begin{array}{l}\text { 5'cattacggtcaatccgecgtttgttcccacggagaatccgTAATAAGTAAttgttact } \\
\text { cgctcacatttaatgttgatgaaagctggcta }\end{array}$ \\
\hline 40nt- lead-lacZ & $\begin{array}{l}\mathbf{5} \text { 'tagccagctttcatcaacattaaatgtgagcgagtaacaaTTACTTATTAcggattct } \\
\text { ccgtgggaacaaacggcggattgaccgtaatg }\end{array}$ \\
\hline 40nt- lag-rps $L$ & $\begin{array}{l}\text { 5'gacgcacacgagctactttacgtagtgcagagttaggtttCCGTggggtagttgtgtacac } \\
\text { acgtgtacaaacaccacgcttttgt }\end{array}$ \\
\hline 40nt- lead-rpsL & $\begin{array}{l}\text { 5'acaaaagcgtggtgtttgtacacgtgtgtacacaactaccccACGGaaacctaactetgc } \\
\text { actacgtaaagtagctcgtgtgcgtc }\end{array}$ \\
\hline $\begin{array}{l}\text { 40nt-lag } \\
\text { SAMArpsLK43R }\end{array}$ & $\begin{array}{l}\text { 5'aacgcacacgagctactttacgcagtgcagagtttggtttCCGTggggcggtggtgtaca } \\
\text { cacgagtacaaacaccacgcttctgt }\end{array}$ \\
\hline
\end{tabular}

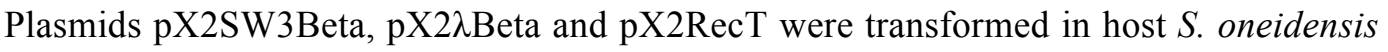
cells JG2150 or JG274 or S. amazonensis cells (JG98), followed by plating on LB + Km and growth at $30{ }^{\circ} \mathrm{C}$. The resulting strain cultured on $\mathrm{LB}+\mathrm{Km}$ was supplemented with arabinose $(20 \mathrm{mM})$ to express the recombinase to $\mathrm{OD} \simeq 0.4-0.5$. Cells were made electrocompetent using sorbitol $(1 \mathrm{M})$ as wash buffer and incubated with $2.5 \mu \mathrm{g}(5 \mu \mathrm{L})$ of ssDNA for three min at room temperature prior to electroporation. After electroporation at $12 \mathrm{kV} / \mathrm{cm}$, cells were allowed a $30 \mathrm{~min}$ ( $2 \mathrm{~h}$ for $\mathrm{rpsL}$ recombineering) recovery shacking at $30{ }^{\circ} \mathrm{C}$ prior to plating. For the experiments at lower temperatures, the same procedure as ice-chilled electrotransformation was followed after keeping the cells on ice for 15 or $60 \mathrm{~min}$.

Following electroporation and the recovery period, aliquots were diluted $10^{4}$-fold on LB medium and all cells of this dilution were spread on $\mathrm{LB}+\mathrm{Km}(50 \mu \mathrm{g} / \mathrm{mL})$ and X-gal $(60 \mu \mathrm{g} / \mathrm{mL})$ agar plates to determine $l a c Z$ cells. To assess the frequency of $r p s L K 43 \mathrm{R}$ mutants, $100 \mu \mathrm{L}$ of a range of 10 -fold serial dilutions were plated on $\mathrm{LB}+\mathrm{Km}(50 \mu \mathrm{g} / \mathrm{mL})$ and Spect $(50 \mu \mathrm{g} / \mathrm{mL})$ agar plates at appropriate dilutions to yield single colonies, while viable cells were determined from colonies growing on $\mathrm{LB}+\mathrm{Km}(50 \mu \mathrm{g} / \mathrm{mL})$ plates. A reverse primer specific to the mutation introduced (lacZ-mut-R or rpsL-mut-R) was utilized for screening by colony PCR prior to sequencing. 


\section{CHAPTER IV:}

\section{EFFICIENT AND PRECISE GENOME EDITING IN SHEWANELLA THROUGH RECOMBINEERING AND CRISPR/CAS9-MEDIATED COUNTERSELECTION}

This chapter was adapted from "Efficient and Precise Genome Editing in Shewanella with Recombineering and CRISPR/Cas9-mediated Counter-selection", submitted in ACS Synthetic Biology, in 2019.

\subsection{Introduction}

The establishment of ssDNA recombineering has been a major advance for Shewanella genome-engineering, but somewhat limited in application in the absence of a robust method for counter-selection against unedited cells. In other bacteria, CRISPR (clustered regularly interspaced short palindromic repeats)- Cas (CRISPR associated) systems have been adopted as a counterselectable marker combined with recombineering, and this combination has proven to be a powerful technology for extremely efficient genome engineering (Yan et al. 2017; Jiang et al. 2013; Ronda et al. 2016). CRISPR and their associated Cas proteins function as an RNA-guided adaptive immune system that allows bacteria and archaea to defend against invading nucleic acids. The CRISPR loci consist of arrays of short repetitive sequences separated by short hypervariable sequences (spacers), which match regions of viruses and plasmids, and thus represent a recording of previous encounters with these foreign genetic elements. Transcription of a CRISPR array by the bacterial RNA polymerase yields a long transcript, the pre-crRNA, which is subsequently cleaved at the repeated sequences and processed to form specific short mature CRISPR RNAs, crRNAs. Primarily via RNA-DNA binding, these crRNAs guide the associated Cas machinery to cleave a specific region of the target foreign DNA known as protospacer, which is complementary to the spacer sequence. Many CRISPR/Cas systems require the presence of a short 2 to $7 \mathrm{nt}$ motif, the protospacer-adjacent motif (PAM), juxtaposed to the targeting region of the intruder DNA. The existence of the PAM site allows tolerance to self by preventing autoimmunity against the spacers from the CRISPR array they encode. Cas-mediated double-stranded DNA (dsDNA) cleavage then 
results in degradation of the attacker's genome and thus immunity (Peters et al. 2015; Selle \& Barrangou 2015).

There are three major groups of CRISPR/Cas systems, categorized as Type I, II and III, which differ in the nature of the cas genes and the mechanism of immunity (Rath et al. 2015; Makarova et al. 2012). Type II is the most widely used in genome engineering due to its inherent simplicity. Type I and III CRISPR systems are more complex, requiring multiple Cas proteins to induce the cleavage of their target foreign DNA. In contrast, Type II systems rely on a simpler mechanism, requiring only the crRNA-guided endonuclease Cas9 and an additional trans-encoded small RNA (tracRNA) for activity, making it a highly suitable tool for genome-editing.

Most studies have used the Streptococcus pyogenes Cas9 (SpCas9) Type II system, in which a chimeric single guide RNA (sgRNA) has been created to mimic the natural two RNA duplexes (Jiang \& Marraffini 2015; Garst et al. 2016; Reisch \& Prather 2015; Ronda et al. 2016). This sgRNA contains a user-programmable 20nt unique spacer (guide sequence) complementary to the target allele of choice, and the SpCas9 binding scaffold to form the sgRNA-SpCas9 ribonucleoprotein complex (Jinek et al. 2012). The first 8-12nt at the 3'- end of the guide are known as the 'seed' sequence; mutations within this region abrogate Cas9 binding activity, while mutations outside the 'seed' can be tolerated (Jinek et al. 2012; Jiang et al. 2013). In addition to the sgRNA, SpCas9 requires the PAM site sequence, NGG, located at the 3' end of the DNA target (20nt target-NGG) to enable Cas9-mediated recognition of the DNA target and creation of a double-strand break (DSB). SpCas9 can also recognize the nt sequence NAG as PAM, albeit with much lower efficiency (Jiang et al. 2013). The PAM site is not included in the targeting sequence used for the sgRNA. Cas9 nucleases cleave DNA through the combined activity of their RuvC and HNH domains, each of which nicks one strand of DNA to generate a blunt-ended DSB. Complete Cas9-mediated cleavage of the bacterial chromosome is usually lethal, since most bacteria lack endogenous nonhomologous end joining (NHEJ) activity (Peters et al. 2015; Selle \& Barrangou 2015). Thus, Cas9-mediated killing can be used as a powerful tool to enhance selection of genetic alterations introduced with recombineering by elimination of chromosomes carrying the wild-type (WT) DNA sequence (Peters et al. 2015; Selle \& Barrangou 2015). However, care should be taken to design spacers with high specificity, since Cas9 bound to weak targeting spacers fails to cut all copies of the E. coli chromosome, leading to cell survival via RecA-dependent homologous recombination between sister chromosomes (Cui \& Bikard 2016).

Jiang et al. (Jiang et al. 2013) were the first to couple CRISPR/Cas9 to recombineering using the $\lambda$ Red system in $E$. coli. Using Cas9 as a counter-selection, a recombination efficiency of $\simeq 65 \%$ of surviving cells was obtained when introducing a point mutation into the $r p s L$ gene. Since 
then, this hybrid in vivo genetic engineering technology has been successfully transferred to other bacterial hosts including T. citrea (Jiang et al. 2015), L. reuteri (Oh \& Van Pijkeren 2014), C. glutamicum (Sung et al. 2017), P. putida (Aparicio et al. 2018) and S. aureus (Penewit et al. 2018). Some of these studies used either several plasmids or expressed components of the two systems from the bacterial chromosome. Even though these strategies allow for control over each component of the system, a disadvantage is the limited portability to other hosts. We set out to explore the possibility of combining ssDNA recombineering with CRISPR/Cas9 in Shewanella to enhance our ability to engineer the genome of these bacteria. Here, we describe a simple twoplasmid system that expresses both Cas 9 and the W3 Beta recombinase from one vector and the targeting sgRNA from a second vector. Our system enables precise site-directed mutagenesis of the bacterial chromosome at a high efficiency ( $>90 \%)$ when the recombinogenic ssDNA oligos and the sgRNA plasmid are introduced by co-transformation into the cells harboring Cas9 and W3 Beta. Using this system (Fig 4.1) we have successfully modified several different genes and introduced various types of mutations including individual base changes and net gene deletions in the model strain S. oneidensis MR-1, as well as in S. amazonensis SB2B. This new method not only simplifies genetic manipulation of Shewanella, but will facilitate implementation of high-throughput genomic engineering technologies. 


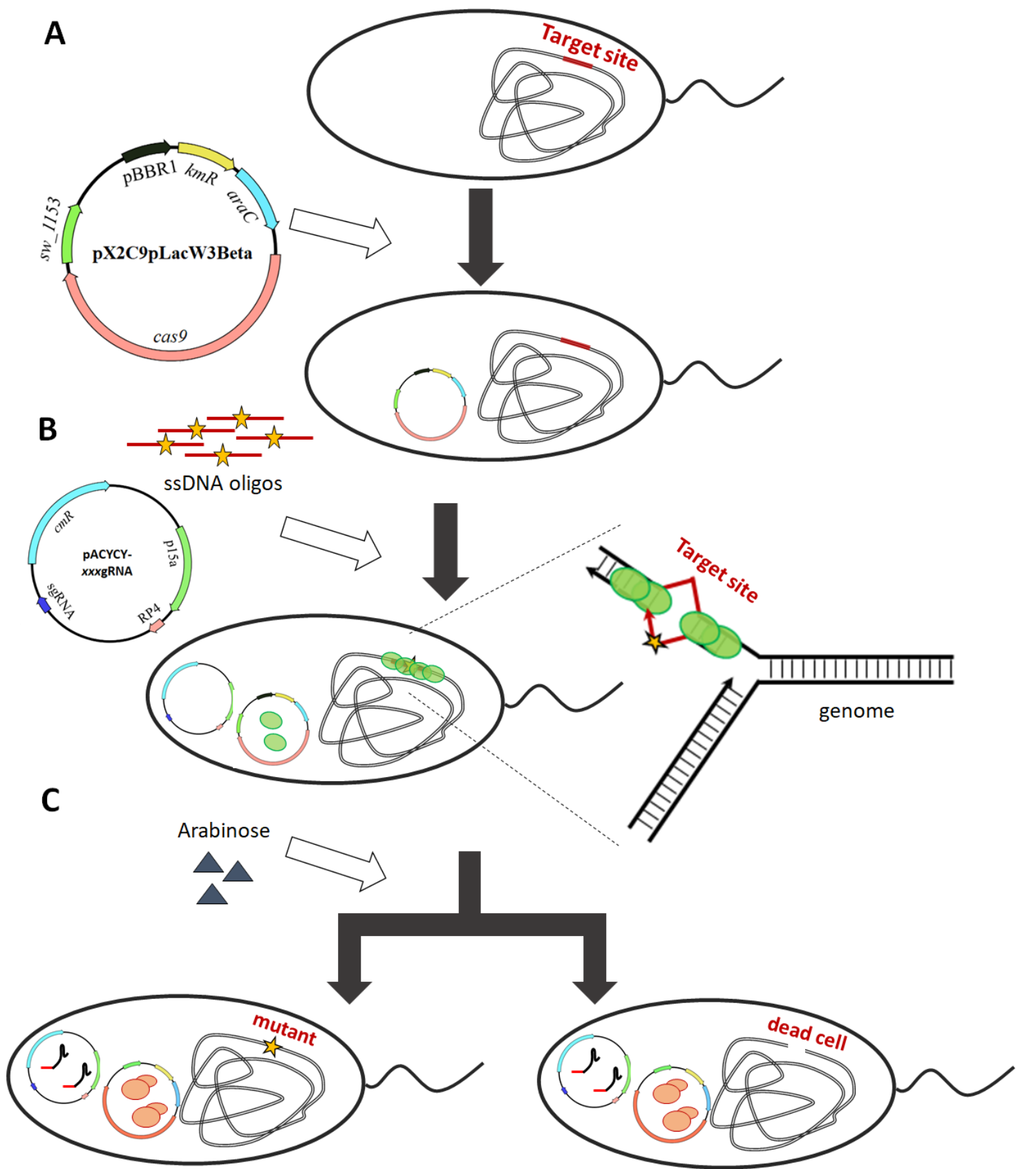

Figure 4. 1. Overview of ssDNA recombineering combined with CRISPR/Cas9 as a counterselection.

The system consists of two plasmids: $\mathrm{pX} 2 \mathrm{C} 9 \mathrm{pLacW} 3 \mathrm{Bet}$ expresses $s p \operatorname{Cas} 9$ from the arabinose inducible $\mathrm{P}_{\mathrm{BAD}}$ promoter, regulated by the arabinose repressor ( $\left.\operatorname{araC}\right)$, and the $\mathrm{s} w_{-} 1153$ recombinase from the constitutive $\mathrm{P}_{\text {Lac }}$ promoter; while plasmid pACYC- $x x x \mathrm{gRNA}$ constitutively expresses the gRNA. For genomic engineering, plasmid pX2C9pLacW3Bet is first introduced into host cells by electroporation, and the transformed cells are plated on L Km50 agar medium and incubated at $30^{\circ} \mathrm{C}$. Plasmid-bearing cells are made electrocompetent and the pACYC- $x x x \mathrm{gRNA}$ is co-transformed with the ssDNA oligos to generate targeted mutations. After recovery from electroporation, cells are plated on $\mathrm{L} \mathrm{Km} 25 \mathrm{Cm} 6.5$ agar medium, supplemented with arabinose to induce the Cas9-mediated counter-selection, and incubated at $30^{\circ} \mathrm{C}$. Mutations are verified by colony PCR using specific primers followed by sequencing of the PCR product. 


\subsection{Results}

\subsubsection{Lethality of DSB Induced by Active Cas9 in Shewanella}

Initially, we asked whether the model bacterium $S$. oneidensis MR-1 contains any CRISPR array or Cas proteins, using the CRISPRDetect webtool, developed by the Brown group at the University of Otago (Biswas et al. 2016). No CRISPR/Cas system was identified when the program default values were used. We then modified the parameters to look for smaller potential CRISPR arrays, and found a possible array consisting of 2 repeats and one spacer located at position 907,711-907,823 of the chromosome; however, no Cas proteins could be detected. Encouraged by our findings, we sought to harness the engineered CRISPR/Cas9 system from $S$. pyogenes through heterologous expression of the key components; the Cas9 nuclease and synthetic chimeric sgRNAs.

Since a non-specific binding activity associated with overexpression of Cas 9 was detected at high concentrations (Cobb et al. 2014; Huang et al. 2015; Wang et al. 2018; Cui et al. 2018), we modulated expression of Cas9 by placing it under control of the arabinose inducible $\mathrm{P}_{\mathrm{BAD}}$ promoter on the kanamycin $(\mathrm{Km})$ resistant pX2Cas9 plasmid (Garst et al. 2016). As a control we used a defective Cas9 (dCas9) from the pdCas9 plasmid (Garst et al. 2016), which binds the target DNA sequence but is unable to cleave. S. oneidensis cells harboring active Cas9 displayed a small decrease in cell viability at high arabinose concentration ( $\simeq 4$-fold at $5 \mathrm{mM}$ arabinose) (Figure 4.2 ), thus low arabinose concentrations were required. No decrease in viability was observed with inactive Cas 9 at any of the concentrations tested. 


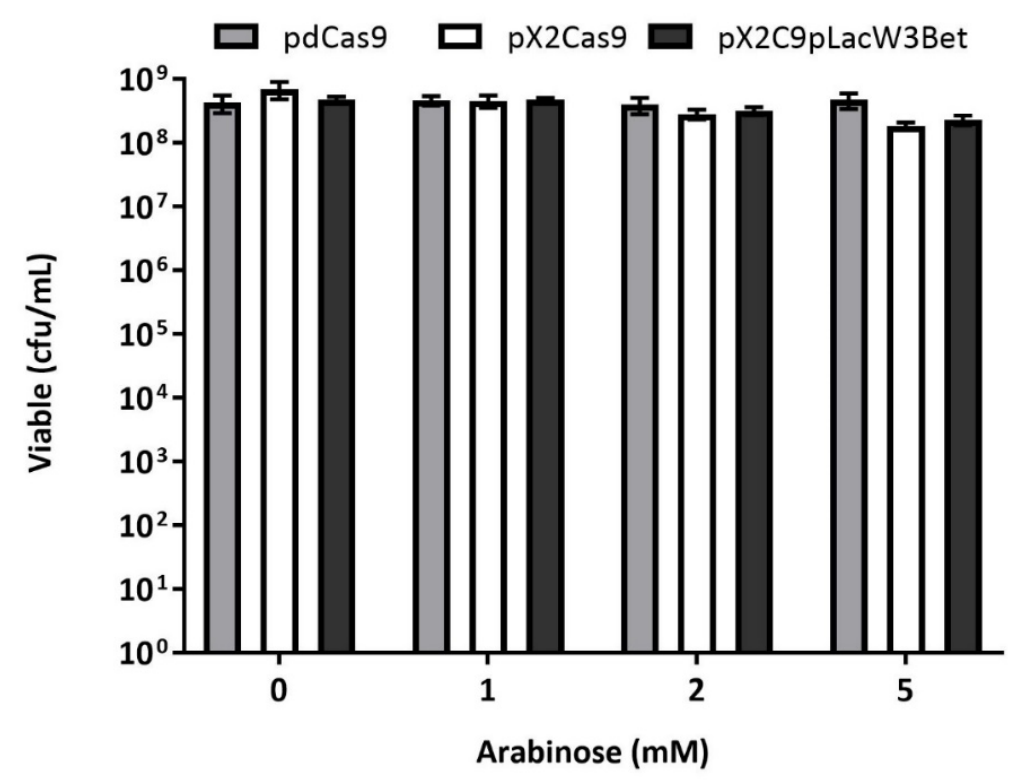

Figure 4. 2. Effect of Cas9 expression on S. oneidensis growth.

Cells were grown to early exponential phase and induced with different arabinose concentrations for 3 hours prior to plating. After induction, different diluted aliquots were spot plated on Km plates and incubated at $30^{\circ} \mathrm{C}$ for $\sim 24$ hours. The error bars represent standard error from three independent experiments.

To assess the specific lethality of Cas9-induced DSB in S. oneidensis, we expressed different combinations of the CRISPR/Cas components. We designed sgRNAs targeting several genes on the S. oneidensis chromosome (Table 4.7 and 4.8). The non-essential genes lacZ, exeM (encoding an extracellular endonuclease) and $m t r A$ (encoding a periplasmic $c$-cytochrome from the Mtr pathway) were chosen, since mutations that suppress these functions are not detrimental to cell growth under aerobic conditions. S. oneidensis MR-1 does not contain an endogenous lacZ gene, thus, we used a strain with the E. coli lacZ gene integrated in single copy on the bacterial chromosome (Corts et al. 2019), which allowed for rapid quantitation of genome editing events. Cells harboring active Cas9 or dCas9 were electroporated with the chloramphenicol resistant (CmR) sgRNA expressing plasmid, pACYC- $X X X \mathrm{gRNA}$. For each of these genes, when both Cas9 and a sgRNA were co-expressed, a large decrease in cell viability was observed. More than $99 \%$ of the cells were killed, resulting in $\simeq 10^{2}-10^{3}$ transformants $/ \mathrm{mL}$ compared to $\simeq 10^{6}$ transformants $/ \mathrm{mL}$ obtained when Cas9 was not induced (Figure $4.3 \mathrm{~A}$ ). A background of $\mathrm{CmR}$ colonies appeared on the $\mathrm{Km} 25 \mathrm{Cm} 6.5$ agar plates, at a rate of $\simeq 10^{1}$ total colonies $/ \mathrm{mL}$ (Figure 4.3A, -DNA control), as observed previously (Groh et al. 2007). Increasing the concentration of $\mathrm{Cm}$ up to $15 \mu \mathrm{g} / \mathrm{mL}$ did not enhance selection but proved detrimental for the cells. Use of a drug marker other than chloramphenicol may enhance editing efficiency in $S$. oneidensis. The rest of the 
colonies surviving the DSB are probably cells that acquired mutations in the cas 9 gene itself or that allow evasion of Cas9 targeting (Garst et al. 2016; Ronda et al. 2016; Stout et al. 2018).
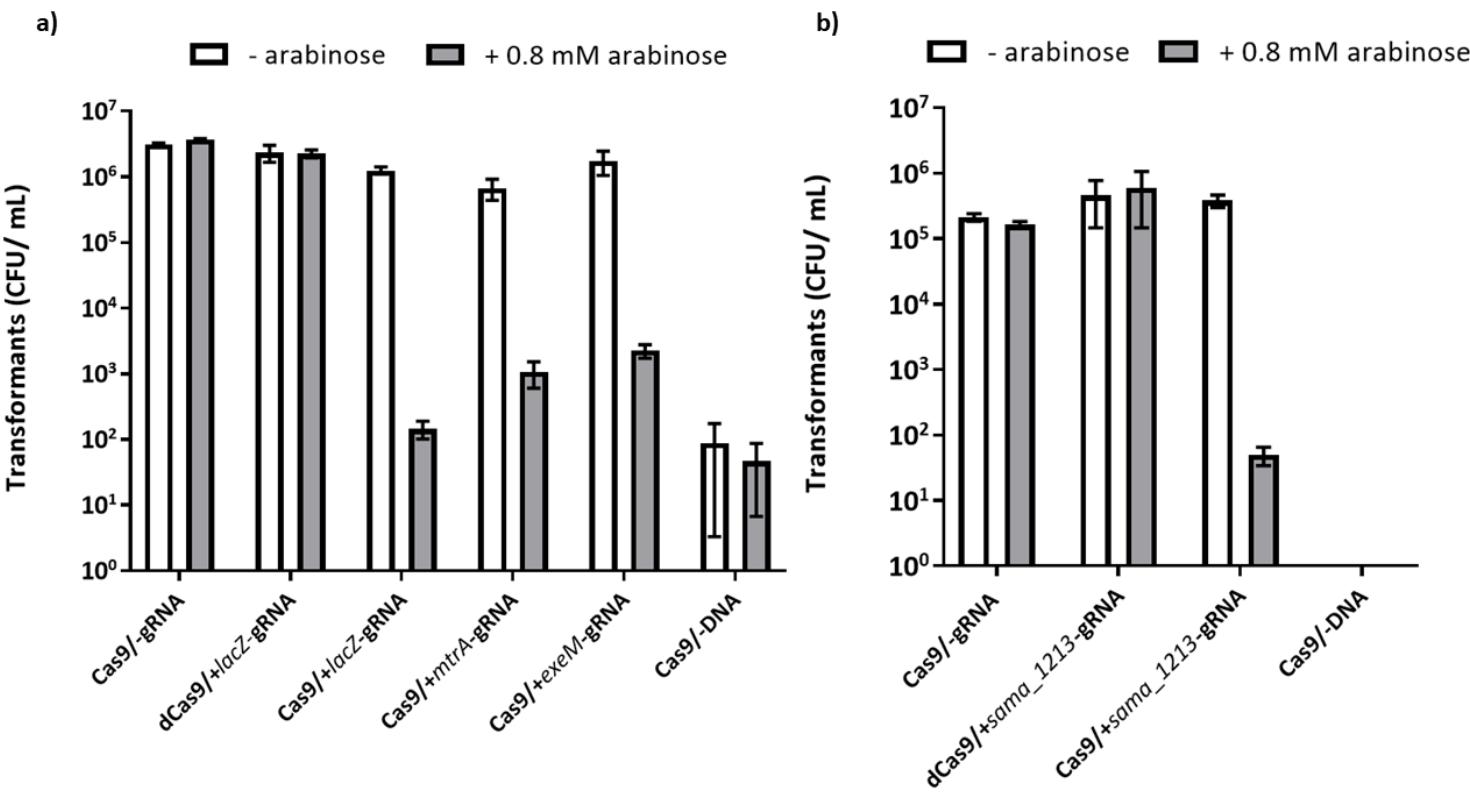

Figure 4. 3. Lethality of DSBs induced by active Cas9 in Shewanella.

a) $S$. oneidensis, b) S. amazonensis. Results were determined as total colony-forming units (CFU) on $\mathrm{L}$ agar plates containing both antibiotics, $\mathrm{Km}$ and $\mathrm{Cm}$, supplemented with or without arabinose. Data are averages of three independent experiments; error bars indicate standard deviation.

In order to evaluate the power of this in vivo genetic engineering technology in another Shewanella species, we assessed CRISPR/Cas9 activity in S. amazonensis SB2B. We designed a sgRNA to target sama_1213, which encodes for the decaheme cytochrome $c$ MtrD. Using the same conditions for $S$. oneidensis, more than 99\% killing was observed in S. amazonensis SB2B, resulting in $\simeq 50$ transformants $/ \mathrm{mL}$ compared to $\simeq 10^{5}$ transformants $/ \mathrm{mL}$ without CRISPR/Cas9 induction (Figure 4.3B). S. amazonensis appears to be more sensitive to chloramphenicol than $S$. oneidensis, since no spontaneous colonies were observed on the selective plates containing $\mathrm{Km} 25$ and Cm6.5 (Figure 4.3B, -DNA control). As controls, we tested variants deficient in Cas9 cutting, using dCas9 (provided from pdCas9) or an empty vector lacking sgRNA (pACYC'). Omitting either part of the CRISPR/Cas9 system, Cas9 or sgRNA, eliminated the capacity of Cas9 to be used as a counter-selection in both strains tested, since the transformation efficiency was not reduced even when induced. This inactivity stands in contrast to the successful CRISPR/Cas9 counterselection we observe when both active Cas9 and sgRNA are expressed (Figure 4.3). 


\subsubsection{Recombinase-independents ssDNA genome editing coupled to CRISPR/Cas9 counter- selection}

In chapter III, we found that $S$. oneidensis can naturally recombine ssDNA without the addition of phage-encoded proteins, albeit at a low frequency of $\simeq 10^{4}$ recombinants $/ 10^{8}$ viable cells, as had been observed in other hosts (Bryan \& Swanson 2011; Swingle, Markel, et al. 2010). Thus, we asked whether we could use CRISPR/Cas9 as a counter-selection to eliminate the WT copies of a gene after recombination in the absence of any phage recombinase expression, as this would provide a very simple genome-editing tool (Figure 4.4). We used 170nt long recombinogenic ssDNA donor templates to introduce ten consecutive base pair (bp) changes within the gene open reading frame (ORF), which resulted in 10bp mutations coupled with a frameshift (Table 4.6). The oligos contain the centered mutations flanked by 80nt homology arms. These mutations also introduce a change in the PAM site, to avoid retargeting by Cas9. We targeted lacZ in S. oneidensis using an oligo complementary to the lagging strand template, since it has been shown in other bacteria that oligos annealing to the lagging strand template results in higher efficiencies (Swingle, Markel, et al. 2010). The $l a c Z$ gene was used because loss of gene function is easily screened for using X-gal supplemented plates, where colonies capable of metabolizing X-gal via activity of the lac $Z$ gene product, $\beta$-galactosidase, turn blue. Different ratios of sgRNA/ssDNA were evaluated, as well as different recovery times after electroporation (Figure 4.5). The optimal result was obtained when $250 \mathrm{ng}$ of sgRNA plasmid and $1 \mu \mathrm{g}$ of ssDNA were co-transformed (ratio 1/4) and the cells were recovered for 4 hours prior to applying the CRISPR/Cas9-based counter-selection. Under these conditions, an efficiency of $\simeq 80 \%$ mutants among total transformed cells was achieved, as confirmed by colony PCR and sequencing (Figure 4.6). Although the frequency of modified cells is the same as in our previous study (Corts et al. 2019) lacking CRISPR/Cas9, $\simeq 10^{-}$

${ }^{4}$ recombinants/total cells, the efficiency increased $\simeq 2 \times 10^{4}$-fold with the addition of the CRISPR system, due to the Cas9-mediated counter-selection. Given that CRISPR systems are broadly active in bacteria, this approach is likely to be generally useful in other species that display a low level of recombinase-independent ssDNA recombination and for which no phage mediated recombination system has been developed. 


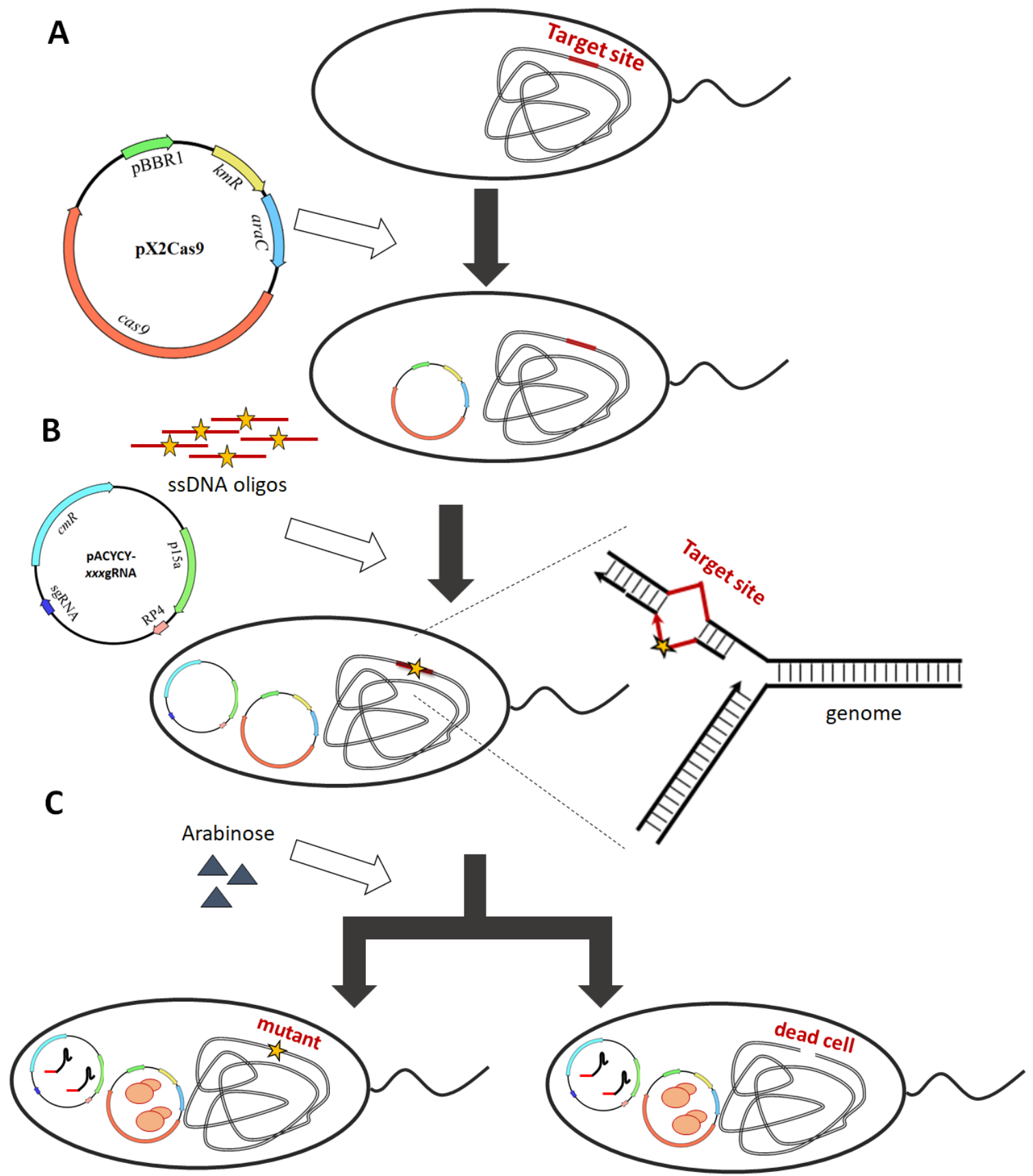

Figure 4. 4. Overview of CRISPR/Cas9 counter-selection coupled with recombinaseindependent ssDNA genome-editing.

The system consists of two plasmids, pX2Cas9, expressing SpCas9 under the inducible arabinose promoter which is regulated by the arabinose repressor (araC), and the pACYC-xxxgRNA, expressing the gRNA to target the gene of interest. Plasmid pX2Cas9 is first introduced into host cells by electrotransformation, followed by plating on $\mathrm{LB}+\mathrm{Km}$ and growth at $30^{\circ} \mathrm{C}$. The resulting cells are made electrocompetent and ssDNA oligos combined with plasmid pACYC- $x x x \mathrm{gRNA}$ are co-transformed by electroporation to generate targeted mutations. After recovery, the cells are plated on $\mathrm{LB}+\mathrm{Km}+\mathrm{Cm}$, supplemented with arabinose to induce the Cas9-mediated counterselection, and incubated at $30^{\circ} \mathrm{C}$. Mutations are verified by colony PCR with specific primers. 

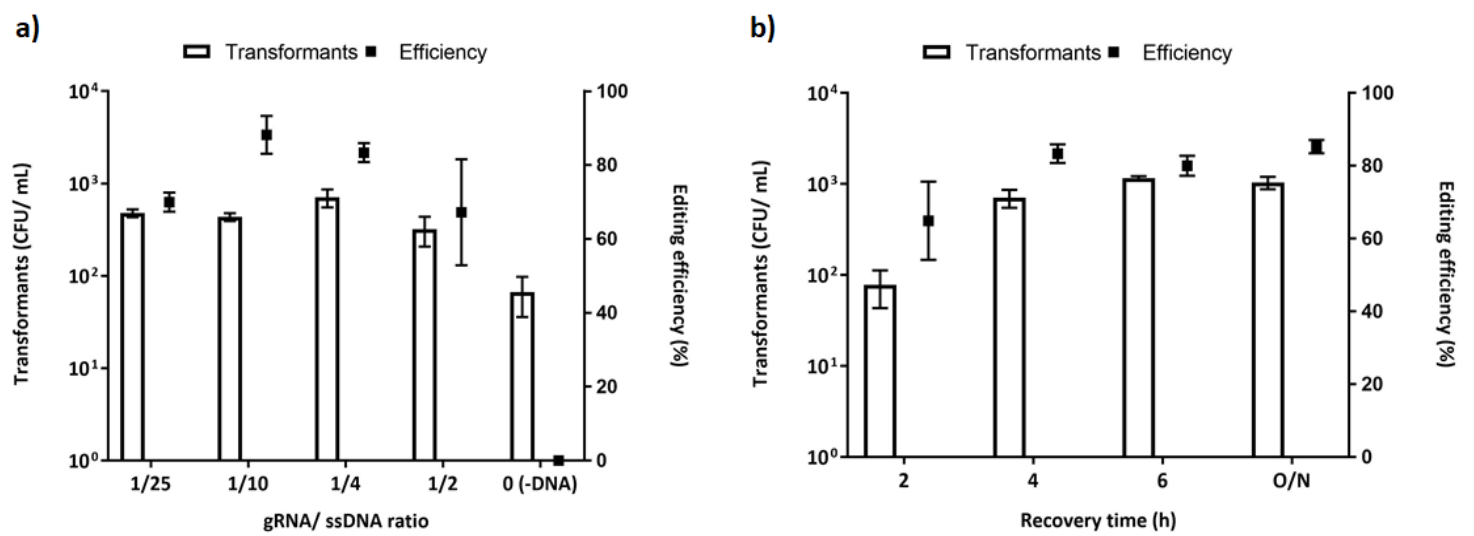

Figure 4. 5. Recombinase-independent ssDNA genome-editing coupled to CRISPR/Cas9 counter-selection.

Genome-editing of $l a c Z$ a) using different ratios of sgRNA to ssDNA with a 4 hour recovery, $b$ ) using a ratio of sgRNA plasmid/ssDNA of $1 / 4$, but varying recovery times after electroporation. Results were determined as total CFU growing on $\mathrm{L}$ agar plates containing both $\mathrm{Km}$ and $\mathrm{Cm}$ and supplemented with arabinose. For lacZ modification the solid agar was also supplemented with XGal and efficiency was calculated as the percentage of white (mutant) colonies over the total. Data are the average of three independent experiments; error bars indicate standard deviation.

a)

\section{$\begin{array}{lllllllllllll}1 & 2 & 3 & 4 & 5 & 6 & 7 & 8 & 9 & 10 & 11 & 12 & \emptyset\end{array}$}

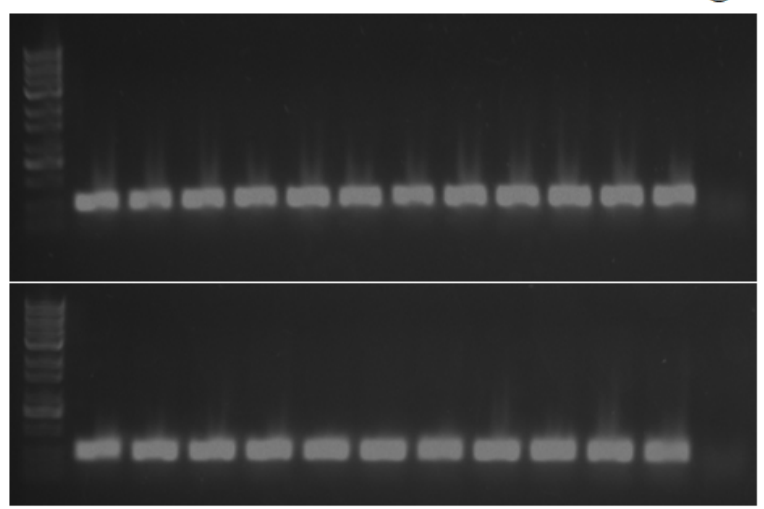

$1314151617181920212223 \quad \emptyset$ b)

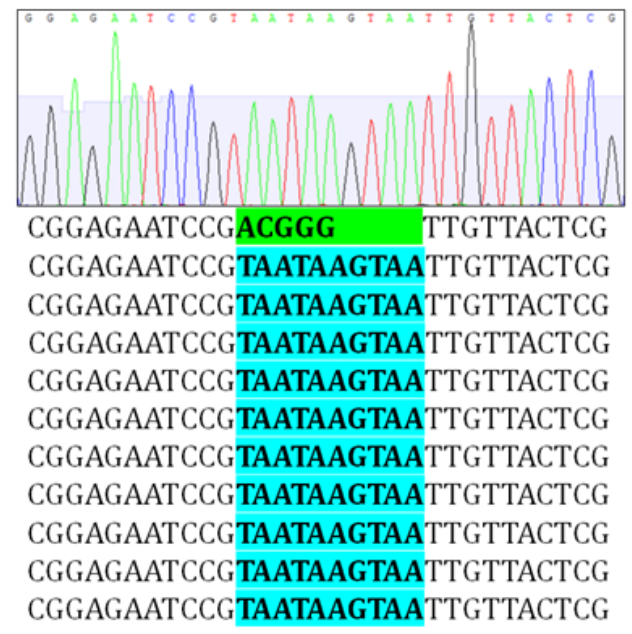

Figure 4. 6. Verification of $l a c Z$ edits in $S$. oneidensis using CRISPR/Cas9 counter-selection coupled with recombinase-independent ssDNA genome-editing.

(a) 23 mutants were screened by colony PCR with a reverse primer specific to the DNA change introduced, validating the correct mutation in all colonies. $\oslash$ indicates a PCR negative control of wild-type lacZ. (b) Sequence confirmation of 10 recombinants identified by white/blue screening, which revealed the mutation of the DNA sequence ACGGG to TAATAAGTAA for all samples, as expected. 


\subsubsection{SsDNA recombineering coupled to CRISPR/Cas9 counter-selection gives high efficiency of genome-editing}

Although the previous method results in a high efficiency of editing and is attractive for its great simplicity, since it requires only the CRISPR/Cas9 system and ssDNA, for large-scale mutagenesis studies, a higher editing efficiency is desired to maximize the number of mutations generated. We previously found that expression of the W3 Beta recombinase in S. oneidensis increases the frequency of recombinant formation $\simeq 10^{3}$-fold when compared to the recombinaseindependent reaction (Corts et al. 2019). Thus, to improve our system, we constructed a vector harboring cas 9 under the arabinose inducible promoter and the gene encoding W3 Beta under a constitutive lac promoter. The resulting plasmid, pX2C9pLacW3Bet editing plasmid (Figure 4.7) was tested for any toxicity effect as we previously did with pX2Cas9. No difference in cell viability was observed when compared to pX2Cas9 plasmid (Figure 4.2).

We used recombinogenic ssDNA donor templates complementary to the lagging strand template (Figure 4.8 and Table 4.6) of the gene of interest, since we previously observed 50- to 100 -fold improved recombineering efficiency with this strand as compared to the leading strand (Corts et al. 2019). We targeted genes lacZ, exeM and mtrA in S. oneidensis and sama_1213 in $S$. amazonensis and used flanking homology arms of different length in the donor ssDNA. Several types of mutations were introduced, including single base changes and whole gene deletions (Figure 4.10 and Table 4.1), to test the robustness of the system. 


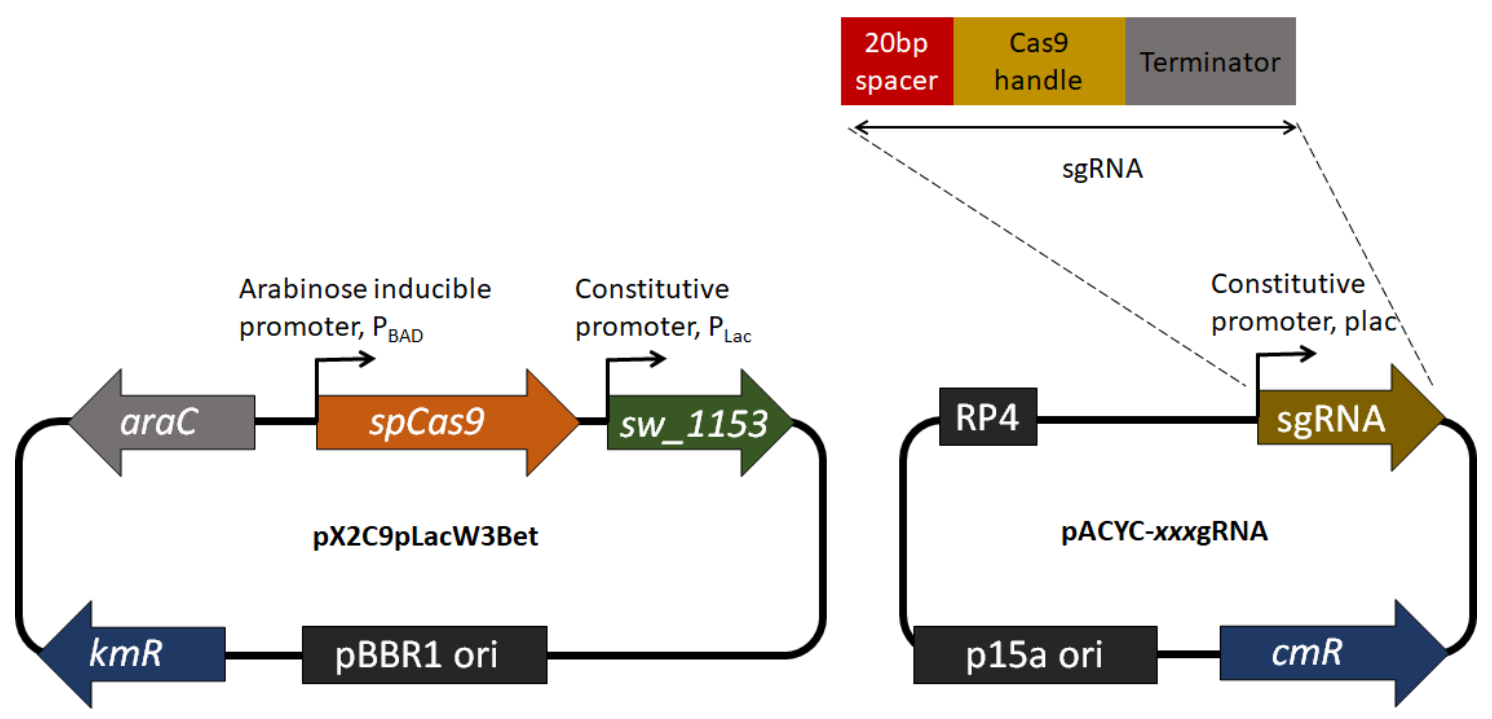

Figure 4. 7. Plasmid maps of the two-plasmid based system.

a) $\mathrm{pX} 2 \mathrm{C} 9 \mathrm{pLacW} 3 \mathrm{Bet}$, consists of the arabinose-inducible $s p C a s 9$ and the constitutively expressed $s w$ 1153. b) The gRNA targeting plasmid, pACYC-xxxgRNA, contains the sgRNA chimera expressed from the constitutive $\mathrm{P}_{\text {Lac }}$ promoter and encompasses the $20 \mathrm{nt}$ region for binding to the DNA target, the Cas9 binding hairpin and a terminator derived from $S$. pyogenes.

a)

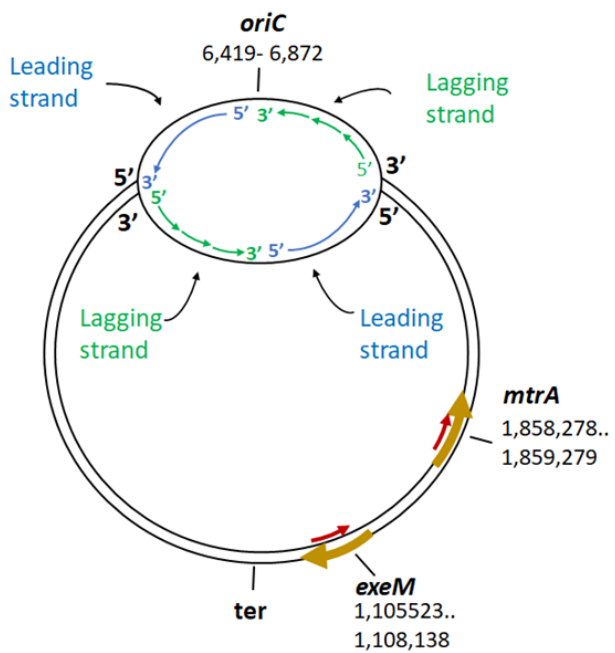

b)

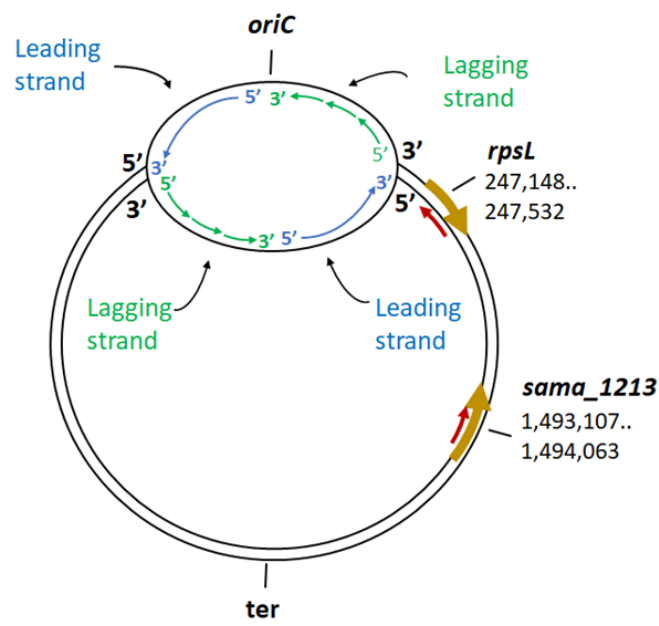

Figure 4. 8. Representation of lagging strand-targeting oligos in $S$. oneidensis MR-1 and $S$. amazonensis SB2B genomes.

(a) Organization of the $S$. oneidensis MR-1 chromosome showing the location of $m$ tr $A$ and exeM genes, and (b) organization of the $S$. amazonensis SB2B chromosome showing the location of sama_1213 and rpsL genes respective to the origin of replication (oriC). The relative position of oriC and terminus define two replichores through which DNA synthesis proceeds. As the oligos mimic Okazaki fragments at the replication fork, the orientation of the target gene in respect to the oriC should be determined in order to obtain optimal recombineering efficiency. Shown in yellow are the genes in the 5'-> 3' direction. Shown in red are the 5 ' $\rightarrow$ 3' alignment of the recombinogenic oligos, which are identical in sequence to the lagging strand and complementary to the lagging strand template of each gene. 
We tested the plasmid pX2C9pLacW3Bet in S. oneidensis by first targeting the lac $Z$ gene and using a 170nt oligo with 80nt flanking homology arms that introduces 10 consecutive bp changes (Table 4.6). Cells harboring the pX2C9pLacW3Bet were co-transformed with the ssDNA and sgRNA plasmid at a ratio $1 / 4$. In contrast to our previous results with the CRISPR/Cas9 system alone, we found that a two hour recovery after electroporation prior to plating on solid agar medium is sufficient to achieve a maximum editing efficiency of $>90 \%$ (Figure 4.9). Thus, we routinely used a two hour recovery period. The number of recombinants was also increased $\_3$-fold when W3 Beta was being expressed (compare Figure 4.5B and 4.9). As previously observed for ssDNA recombineering (Corts et al. 2019), no significant difference in efficiency was found when using 40nt versus $80 \mathrm{nt}$ homology arms to make this 10bp DNA change (Table 4.1). Similarly, an efficiency of $>90 \%$ was obtained when making the same type of mutation in $m t r A$ and exeM in $S$. oneidensis (Table 4.1). All recombinants were verified by colony PCR screen and sequencing (Figure 4.11). Compared to our previous recombineering study in S. oneidensis (Corts et al. 2019), adding the CRISPR/Cas9 counter-selection results in a $\simeq 20$-fold apparent increase in editing efficiency.

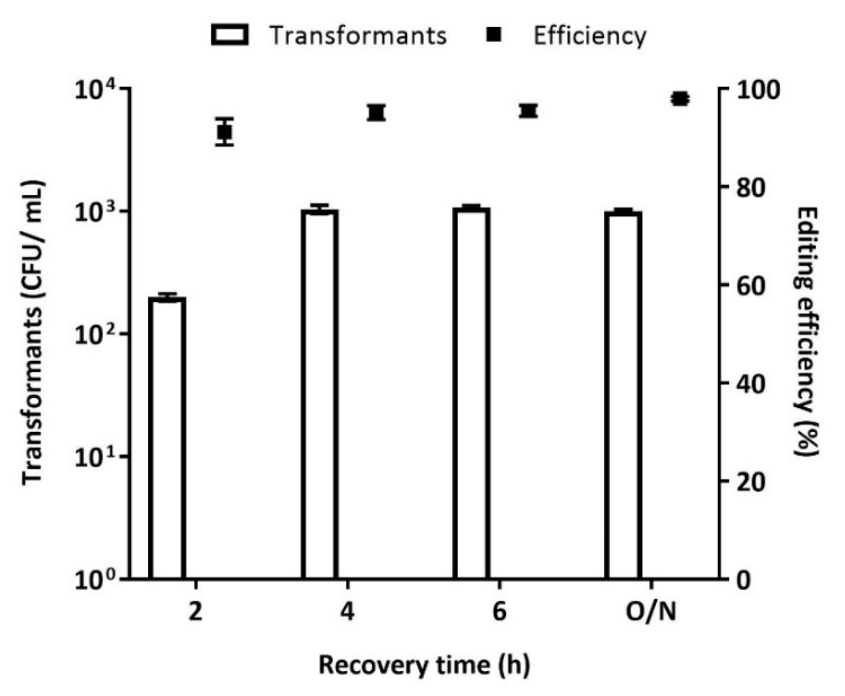

Figure 4. 9. ssDNA recombineering combined with CRISPR/Cas9 as a counter-selection in $S$. oneidensis.

The lacZ gene was edited in $S$. oneidensis using the oligo targeting the lagging strand template with 80nt flanking homology arms. Cells were co-transformed with the gRNA expressing plasmid and ssDNA at a ratio $1 / 4$. After different recovery times at $30^{\circ} \mathrm{C}$, aliquots were diluted and cells were plated on $\mathrm{Km}+\mathrm{Cm}$ selective agar plates supplemented with arabinose at appropriate dilutions to yield single colonies. 
Strikingly, when deleting the entire $m \operatorname{tr} A$ gene, the same high recombination efficiency was obtained using an oligo with 80nt flanking homology arms. However, the efficiency dropped when shorter flanking arms of $60 \mathrm{nt}$ and 40 nt were used, to $\simeq 77 \%$ and $\simeq 8 \%$, respectively (Table 4.1). Thus, although 40nt homology arms is adequate to obtain maximal efficiency when making smaller mutations, longer homologies of $80 \mathrm{nt}$ are required to make larger deletions with high efficiency in $S$. oneidensis. Putative recombinants were verified by colony PCR followed by sequencing (Figure 4.13) and tested for their inability to reduce iron (Figure 4.15), as previously described (Coursolle \& Gralnick 2010). As expected, $m$ tr $A$ deletion mutants were unable to reduce iron citrate.

Sawitzke et al. (Sawitzke et al. 2011) showed that in E. coli most single base pair mismatches as well as insertions or deletions up to three nucleotides in length are corrected by the methyl-directed mismatch repair (MMR) system, which is present in the host. Thus, in order to make a 1 bp mutation in exeM (amino acid 136, TGG>TGA), we also introduced 4 nearby consecutive wobble alterations, which improve the frequency of recombinants in E. coli (Sawitzke et al. 2011) but do not alter the amino acid sequence encoded by the modified gene, and allow creation of a single amino acid change in the desired protein. Interestingly, a marginally lower recombination efficiency of $\_36 \%$ (Table 4.1 ) was obtained when using an oligo with 40nt of homology arms, as opposed to the introduction of 10bp DNA change. This result suggests that this combination of mutations may not be sufficient to entirely escape the host MMR system in $S$. oneidensis. Putative recombinants were verified by colony PCR and sequencing (Figure 4.14). 

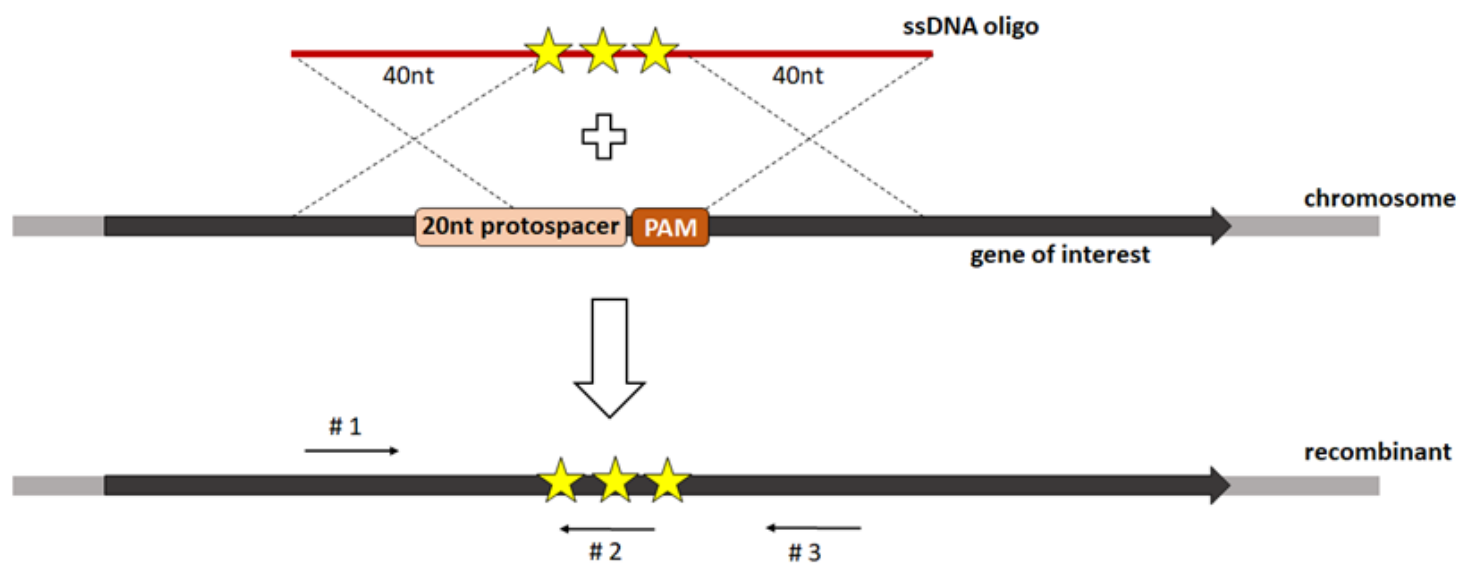

b) Deletions

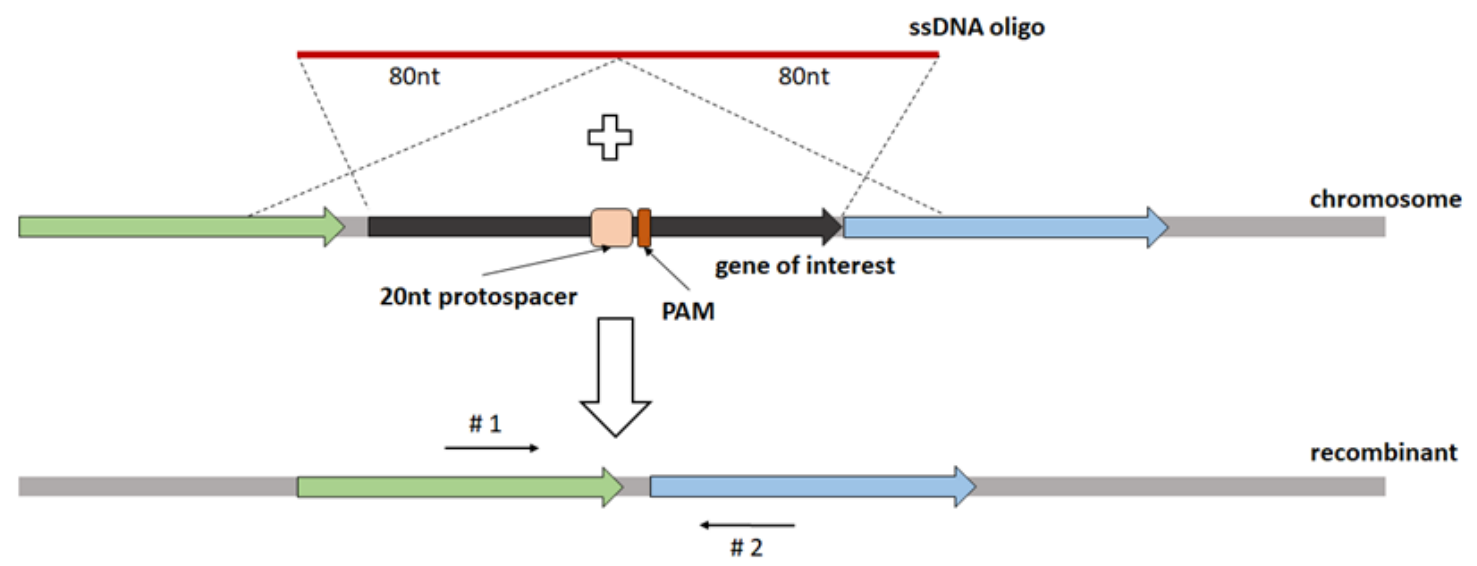

Figure 4. 10. Schematics of the mutations created by ssDNA recombineering coupled to CRISPR/Cas9 counter-selection.

a) For mismatches or small insertions, mutations are introduced at the center of the long oligo and two homology arms are collated on each side. Short (20-24bp) primers are used to confirm the recombinants, as follows: primers \#1 and \#2 are used for colony PCR screening to verify the recombinants, while primers $\# 1$ and $\# 3$ are used to make a PCR product for sequencing confirmation. b) For deletions, the long oligo has homology arms designed to recombine with the DNA sequences flanking the gene to be deleted. Short primers \#1 and \#2 are used for size verification of the recombinants and for sequencing.

We performed the $1 \mathrm{bp}$ point mutation in exeM two times, each in triplicate, using two independently synthesized oligos, both with 40nt homology arms and the same mutations in the center of the oligo. One of the oligos gave the expected results after sequencing of 10 colonies, in agreement with the colony PCR results. While the second oligo gave the same $\backsim 36 \%$ efficiency with the colony PCR screen, but sequencing of 16 colonies showed that $\_40 \%$ of the putative recombinants had additional or different unwanted mutations. These undesired DNA alterations were $1 \mathrm{bp}$ changes, both point mutations and frameshifts, and were located within the region of the 
chromosome where the oligo anneals and recombines. Thus, this oligo resulted in a slightly lower efficiency $(\simeq 22 \%$ ) of correct isolates. As observed by Oppenheim and colleagues (Oppenheim et al. 2004), the unwanted mutations apparently arose during chemical synthesis of the ssDNA oligo. Although finding unwanted mutations is infrequent, it is important to confirm the final construct by sequence analysis, especially the regions encompassed by the oligo. We tried using shorter oligos to minimize the chances of getting these unwanted mutations, however, a lower efficiency of $\simeq 23 \%$ was obtained when using 30 nt flanking homology arms (Table 4.1 ).

Table 4. 1. Genome editing of different genes in $S$. oneidensis and $S$. amazonensis.

Oligos complementary to the lagging strand template of the targeted gene with flanking homologies of different length were used. Cells harboring the editing plasmid pX2C9pLacW3Bet were cotransformed with the sgRNA plasmid and ssDNA at a ratio $1 / 4$ and recovered for 2 hours after electroporation. Transformants were determined as total CFU growing on agar plates with both antibiotics, $\mathrm{Km}$ and $\mathrm{Cm}$, supplemented with arabinose. For lacZ tests, plates were also supplemented with X-Gal. Data shows averages of three independent experiments and error bars depict standard deviations.

\begin{tabular}{|c|c|c|c|c|}
\hline Gene & Mutation & $\begin{array}{l}\text { Oligo homology } \\
\text { arms }\end{array}$ & $\begin{array}{l}\text { Transformants } \\
\text { (CFU/mL) }\end{array}$ & $\begin{array}{l}\text { Efficiency (\% } \\
\text { positive/total) }\end{array}$ \\
\hline \multicolumn{5}{|l|}{ S. oneidensis } \\
\hline \multirow{3}{*}{$\operatorname{lac} Z$} & \multirow{2}{*}{$10 b p$} & $80 \mathrm{nt}$ & $2.00 \times 10^{2} \pm 1.39 \times 10^{1}$ & $91.23 \pm 2.67 \%^{\mathrm{a}}$ \\
\hline & & 40nt & $3.33 \times 10^{2} \pm 5.67 \times 10^{1}$ & $93.96 \pm 2.13 \%^{\mathrm{a}}$ \\
\hline & No oligo control & & $2.67 \pm 0.67$ & $\mathrm{~N} / \mathrm{A}$ \\
\hline \multirow{5}{*}{$m$ trA } & $10 b p$ & $80 \mathrm{nt}$ & $5.82 \times 10^{2} \pm 4.42 \times 10^{1}$ & $93.18 \pm 6.82 \%^{b}$ \\
\hline & \multirow{3}{*}{$\begin{array}{l}\Delta 1002 \text { (gene } \\
\mathrm{KO})\end{array}$} & $80 \mathrm{nt}$ & $3.58 \times 10^{2} \pm 3.10 \times 10^{1}$ & $95.45 \pm 4.55 \%^{\mathrm{b}}$ \\
\hline & & $60 \mathrm{nt}$ & $4.90 \times 10^{1} \pm 9.04$ & $77.27 \pm 4.55 \%^{\mathrm{b}}$ \\
\hline & & 40nt & $4.68 \times 10^{1} \pm 1.55 \times 10^{1}$ & $7.88 \pm 1.69 \%^{b}$ \\
\hline & No oligo control & & $\begin{array}{l}6.51 \times 10^{1} \pm \\
5.08 \times 10^{1}\end{array}$ & N/A \\
\hline \multirow{4}{*}{ exeM } & $10 \mathrm{bp}$ & $80 \mathrm{nt}$ & $2.39 \times 10^{3} \pm 2.58 \times 10^{2}$ & $95.45 \pm 0.00 \%^{b}$ \\
\hline & \multirow{2}{*}{$1 \mathrm{bp}$} & $40 \mathrm{nt}$ & $3.90 \times 10^{2} \pm 4.67 \times 10^{1}$ & $36.36 \pm 4.55 \%^{\mathrm{b}}$ \\
\hline & & 30nt & $7.83 \times 10^{1} \pm 1.85 \times 10^{1}$ & $22.73 \pm 4.55 \%^{\mathrm{b}}$ \\
\hline & No oligo control & & $1.79 \times 10^{2} \pm 6.22 \times 10^{1}$ & N/A \\
\hline \multicolumn{5}{|c|}{ S. amazonensis } \\
\hline \multirow{3}{*}{ sama_1213 } & \multirow[t]{2}{*}{$10 \mathrm{bp}$} & $80 \mathrm{nt}$ & $\begin{array}{l}4.28 \times 10^{2} \pm \\
1.19 \times 10^{2}\end{array}$ & $86.36 \pm 9.09 \%^{b}$ \\
\hline & & 40nt & $2.82 \times 10^{2} \pm 1.56$ & $97.73 \pm 2.27 \%^{b}$ \\
\hline & No oligo control & & $\begin{array}{l}9.51 \times 10^{1} \pm \\
6.18 \times 10^{1}\end{array}$ & N/A \\
\hline
\end{tabular}

N/A: not applicable, ${ }^{a}$ Determined by white/blue screening, as the $\%$ of mutant white recombinant colonies over the total number of transformants. Calculated from three independent experiments. ${ }^{\mathrm{b}}$ Determined by colony PCR screening of more than 20 transformant colonies with specific primers to the mutation introduced (Figure 4.10), as the number of positive recombinants over the total number of colonies tested. Calculated from at least two independent experiments. 
We then wished to assess our system in a different Shewanella strain, S. amazonensis. Following the same protocol as in S. oneidensis, we observed the same efficiency of $>90 \%$ recombinants among total transformed cells when introducing 10 consecutive base changes in the sama_1213 gene, which was further verified by colony PCR screening and sequencing (Figure 4.12). As with $S$. oneidensis, no significant difference in frequency was found whether the ssDNA oligo contained 40nt or 80nt homology arms (Table 4.1). We previously found the W3 Beta recombinase to be also functional in $E$. coli at the same high efficiency as the $\lambda$-Red Beta (Corts et al. 2019), thus our system may be active in other bacterial species as well.

While the results above validate the high potential of this technology in Shewanella, one challenge facing Cas9-DSB mediated counter-selection in bacteria is the possibility of off-targeting by Cas9 (Cui et al. 2018). However, this can be solved by using different approaches recently developed to enhance on-target specificity, such as the use of engineered Cas9 nucleases (Karvelis et al. 2017; Lee et al. 2018) or chemically modified sgRNAs (Ryan et al. 2018). 
a)

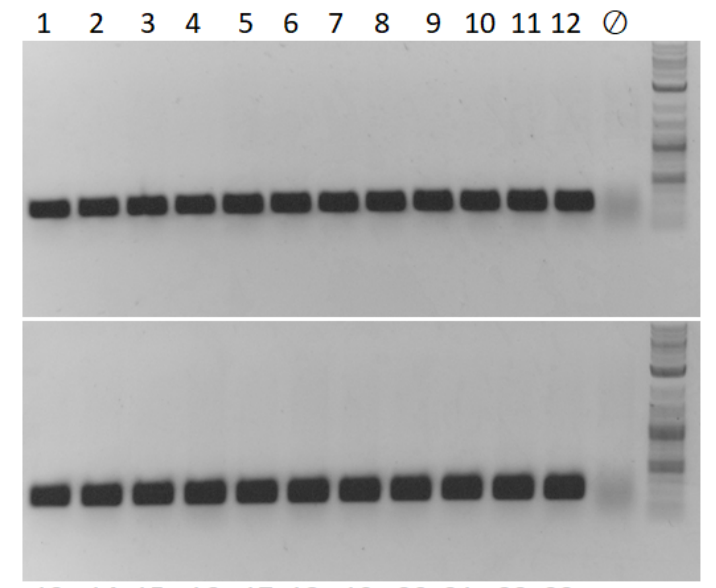

$\begin{array}{llllllllllll}13 & 14 & 15 & 16 & 17 & 18 & 19 & 20 & 21 & 22 & 23 & \emptyset\end{array}$

c)

$\begin{array}{lllllllllllllllll}1 & 2 & 3 & 4 & 5 & 6 & 7 & 8 & 9 & 10 & 11 & 12 & 13 & 14 & 15 & 16 & 0\end{array}$

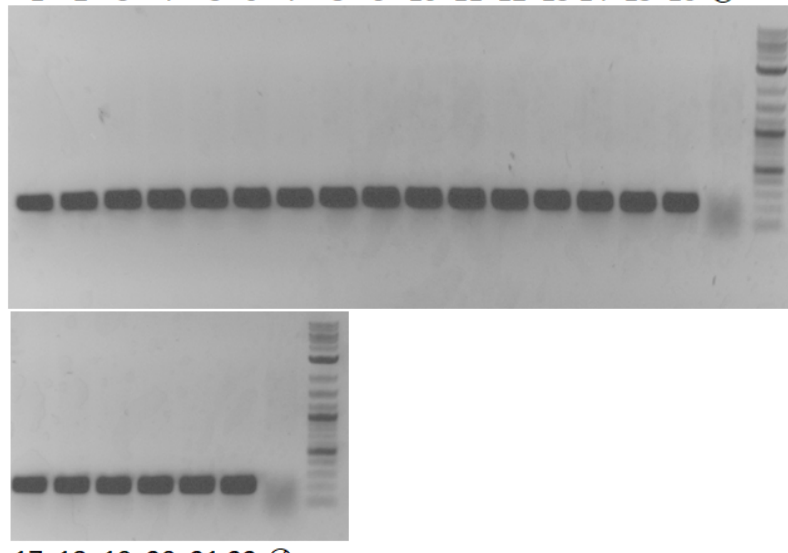

$171819202122 \emptyset$

e)

$\begin{array}{lllllllllllllllll}1 & 2 & 3 & 4 & 5 & 6 & 7 & 8 & 9 & 10 & 11 & 12 & 13 & 14 & 15 & 16 & \emptyset\end{array}$

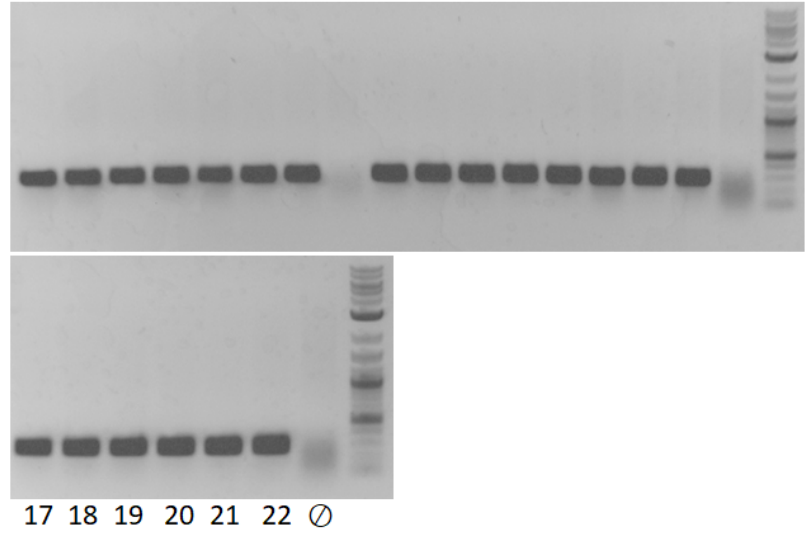

$171819202122 \varnothing$ f)

b)

GGAGAATCCGTAATAAGTAATTGTTACTCG

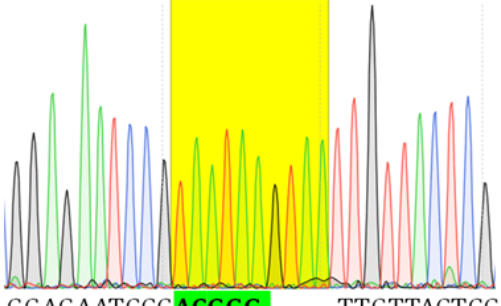

GGAGAATCCGACGGG

TTGTTACTCG

GGAGAATCCGTAATAAGTAATTGTTACTCG GGAGAATCCGTAATAAGTAATTGTTACTCG GGAGAATCCGTAATAAGTAATTGTTACTCG GGAGAATCCGTAATAAGTAATTGTTACTCG GGAGAATCCGTAATAAGTAATTGTTACTCG GGAGAATCCGTAATAAGTAATTGTTACTCG GGAGAATCCGTAATAAGTAATTGTTACTCG GGAGAATCCGTAATAAGTAATTGTTACTCG GGAGAATCCGTAATAAGTAATTGTTACTCG GGAGAATCCGTAATAAGTAATTGTTACTCG

d)

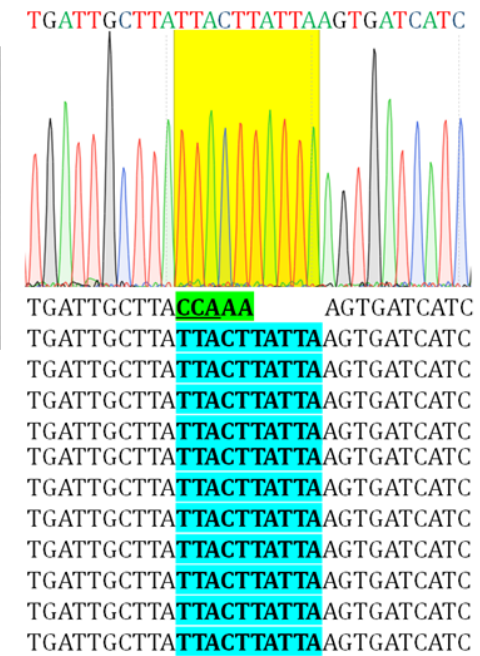

GCCAACCCCTTGATGAGTAAGTTGGGGGCT

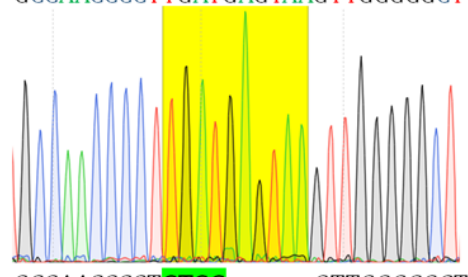

GCCAACCCCTGTGG GTTGGGGGCT GCCAACCCCTTGATGAGTAAGTTGGGGGCT GCCAACCCCTTGATGAGTAAGTTGGGGGCT GCCAACCCCTTGATGAGTAAGTTGGGGGCT GCCAACCCCTTGATGAGTAAGTTGGGGGCT GCCAACCCCTTGATGAGTAAGTTGGGGGCT GCCAACCCCTTGATGAGTAAGTTGGGGGCT GCCAACCCCTTGATGAGTAAGTTGGGGGCT GCCAACCCCTTGATGAGTAAGTTGGGGGCT GCCAACCCCTTGATGAGTAAGTTGGGGGCT GCCAACCCCTTGATGAGTAAGTTGGGGGCT GCCAACCCCTTGATGAGTAAGTTGGGGGCT 
Figure 4. 11. Verification of $10 \mathrm{bp}$ lac $Z$, $m$ tr $A$ and exeM mutants by recombineering combined with CRISPR/Cas9 counter-selection.

(a) 23 lacZ mutants identified by white/blue screening were screened by colony PCR with a reverse primer specific to the DNA change introduced, validating the correct mutation in all colonies. (b) Sequence confirmation of 10 recombinants identified in (a), which revealed the mutation of the DNA sequence 5'-ACGGG-3' to 5'-TAATAAGTAA-3' for all samples. (c) $22 \mathrm{mtrA}$ putative mutants were screened by colony PCR with a reverse primer specific to the DNA change introduced, validating the correct mutation in all colonies. (d) Sequence confirmation of 10 recombinants identified in (c), which revealed the mutation of the DNA sequence 5'-TTTGG-3' to 5'-TAATAAGTAA-3' for all recombinants. (e) 22 exe $M$ putative mutants were screened by colony PCR with a reverse primer specific to the DNA change introduced, validating the correct mutation in 21 colonies out of the total 22 screened. (f) Sequence confirmation of 10 recombinants identified in (e), which revealed the mutation of the DNA sequence 5'-GTGG-3' to 5'-TGATGAGTAA-3' for all recombinants. $\oslash$ indicates a PCR negative control of the wild-type gene. The PAM sequence 5'-NGG-3' is underlined. Oligos with 80nt of flanking homology arms were used. 
b)

a)

$\begin{array}{lllllllllllllllllll}1 & 2 & 3 & 4 & 5 & 6 & 7 & 8 & 9 & 10 & 11 & 12 & 13 & 14 & 15 & 16 & \varnothing\end{array}$
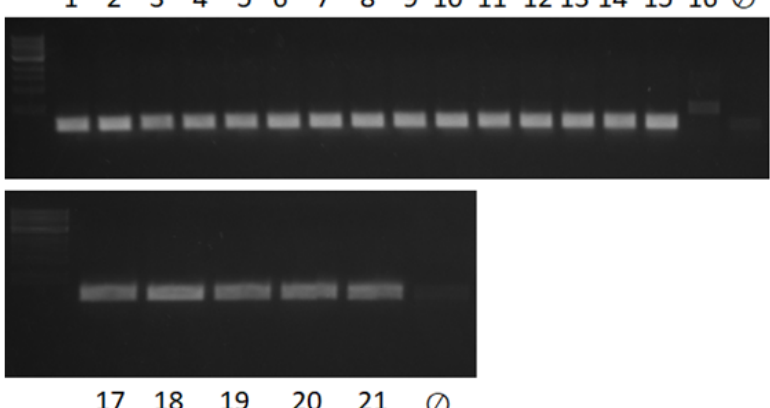

$\begin{array}{llllll}17 & 18 & 19 & 20 & 21 & \emptyset\end{array}$

c)

$\begin{array}{lllllllllllllll}1 & 2 & 3 & 4 & 5 & 6 & 7 & 8 & 9 & 10 & 11 & 121314 & 15 & 160\end{array}$

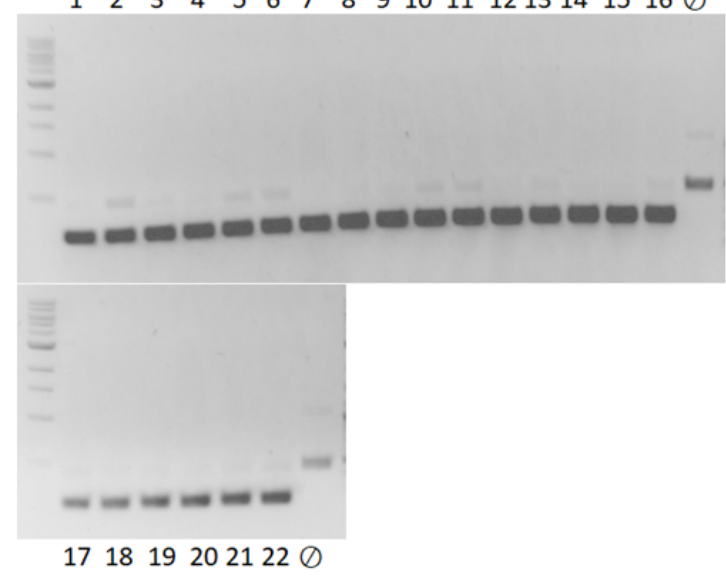

GCTTGTGGATTTACTTATTACCTTTTGTTC

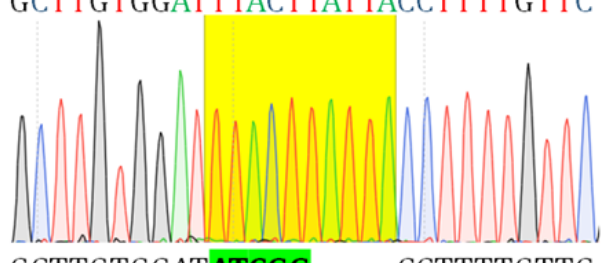

GCTTGTGGATATCGG CCTTTTGTTC GCTTGTGGATTTACTTATTACCTTTTGTTC GCTTGTGGATTTACTTATTACCTTTTGTTC GCTTGTGGATTTACTTATTACCTTTTGTTC GCTTGTGGATTTACTTATTACCTTTTGTTC GCTTGTGGATTTACTTATTACCTTTTGTTC GCTTGTGGATTTACTTATTACCTTTTGTTC GCTTGTGGATTTACTTATTACCTTTTGTTC GCTTGTGGATTTACTTATTACCTTTTGTTC

d)

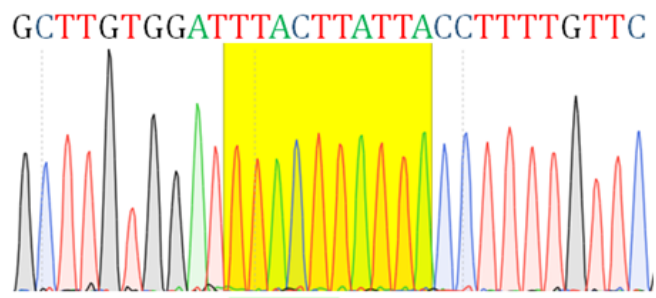

GCTTGTGGATATCGG CCTTTTGTTC GCTTGTGGATTTACTTATTACCTTTTGTTC GCTTGTGGATTTACTTATTACCTTTTGTTC GCTTGTGGATTTACTTATTACCTTTTGTTC GCTTGTGGATTTACTTATTACCTTTTGTTC GCTTGTGGATTTACTTATTACCTTTTGTTC GCTTGTGGATTTACTTATTACCTTTTGTTC GCTTGTGGATTTACTTATTACCTTTTGTTC GCTTGTGGATTTACTTATTACCTTTTGTTC GCTTGTGGATTTACTTATTACCTTTTGTTC GCTTGTGGATTTACTTATTACCTTTTGTTC

Figure 4. 12. Verification of sama_1213 mutants by recombineering combined with CRISPR/Cas9 counter-selection using different size oligos.

(a) 21 putative sama_1213 mutants created using an oligo with 80nt of flanking homology arms were screened by colony PCR with a forward primer specific to the DNA change introduced, validating the correct mutation in 20 colonies. (b) Sequence confirmation of 8 recombinants identified in (a), which revealed the mutation of the DNA sequence 5'-CCGAT-3' to 5'TAATAAGTAA-3' for all samples. (c) 22 putative sama_1213 mutants created using an oligo with 40nt of flanking homology arms were screened by colony PCR with a forward primer specific to the DNA change introduced, validating the correct mutation in all 22 colonies. (d) Sequence confirmation of 10 recombinants identified in (c), which revealed the mutation of the DNA sequence 5'-CCGAT-3' to 5'-TAATAAGTAA-3' for all samples. $\oslash$ indicates a PCR negative control of the wild-type gene. The PAM sequence NGG is underlined. 


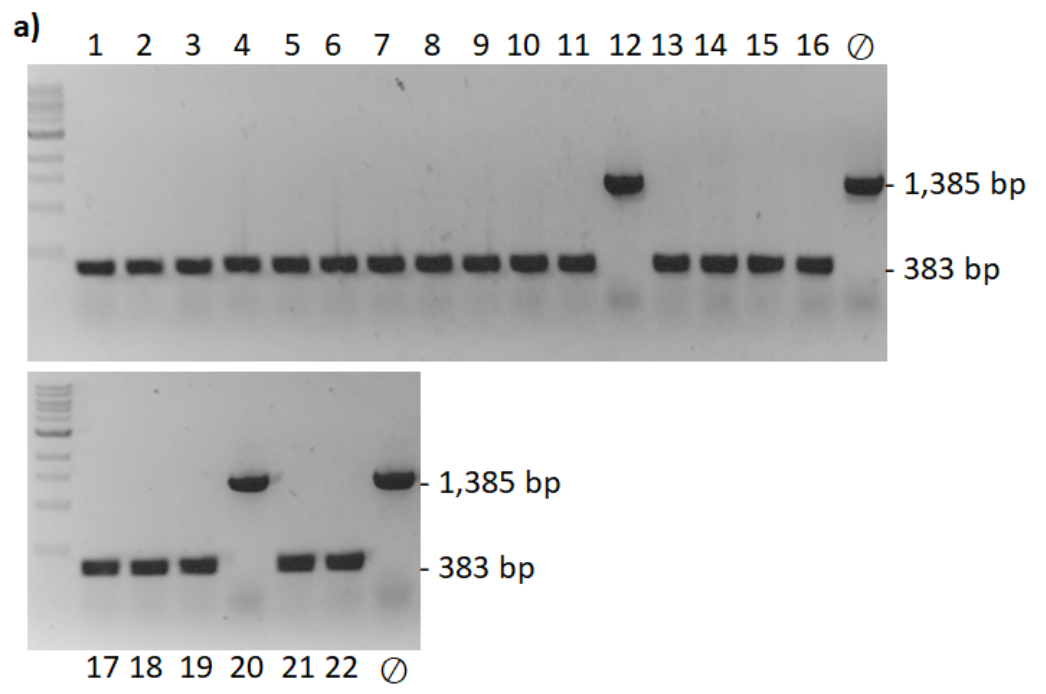

b) $\begin{array}{llllllllllllllll}1 & 2 & 3 & 4 & 5 & 6 & 7 & 8 & 9 & 10 & 11 & 12 & 13 & 14 & 15 & 16\end{array}$

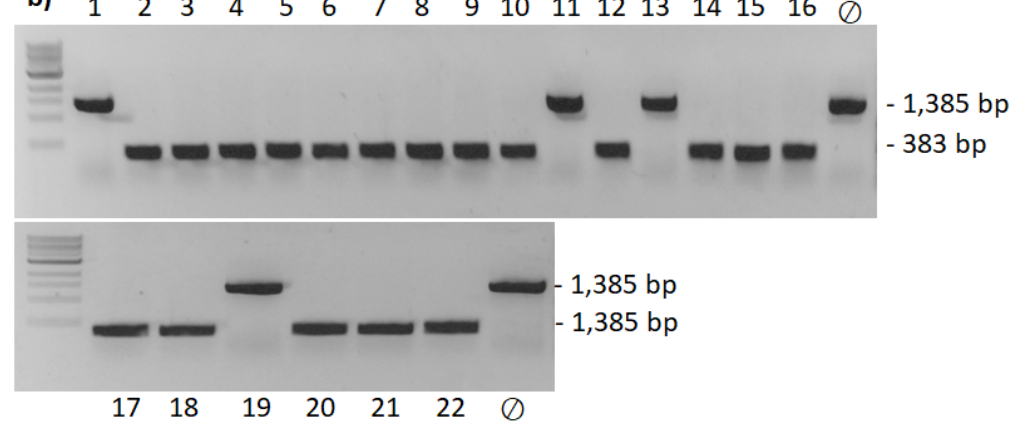

c)

c) $\begin{array}{lllllllllllllllllll}1 & 2 & 3 & 4 & 5 & 6 & 7 & 8 & 9 & 10 & 11 & 121314 & 15 & 16 & \emptyset\end{array}$

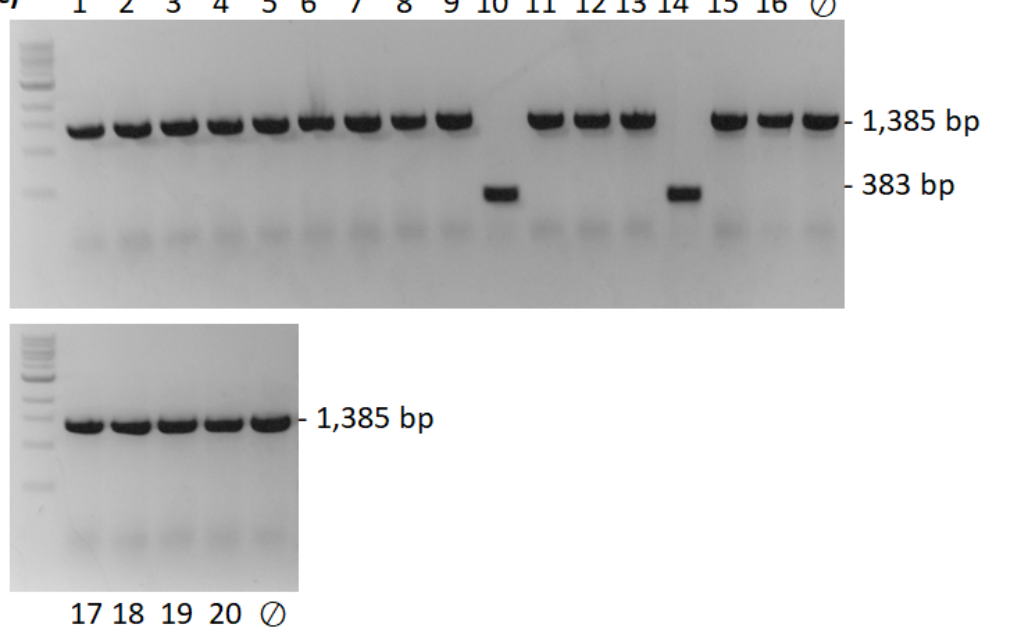

Figure 4. 13. Verification of $m$ tr $A$ deletion mutants by recombineering combined with CRISPR/Cas9 counter-selection using different size oligos.

Several putative mtrA mutants created using an oligo with (a) 80nt, (b) 60nt, and (c) 40nt flanking homology arms were screened by colony PCR with primers binding outside of the mtrA ORF, validating the correct mutation in (a) 20, (b) 18 and (c) 2 colonies, respectively, which showed the correct size of $383 \mathrm{bp}$ after the deletion. 10 of these correct colonies from each experiment (a) and (b), and 5 correct colonies from (c) were further sequenced confirming the right knock out sequence. $\oslash$ indicates a PCR negative control of the wild-type gene. 


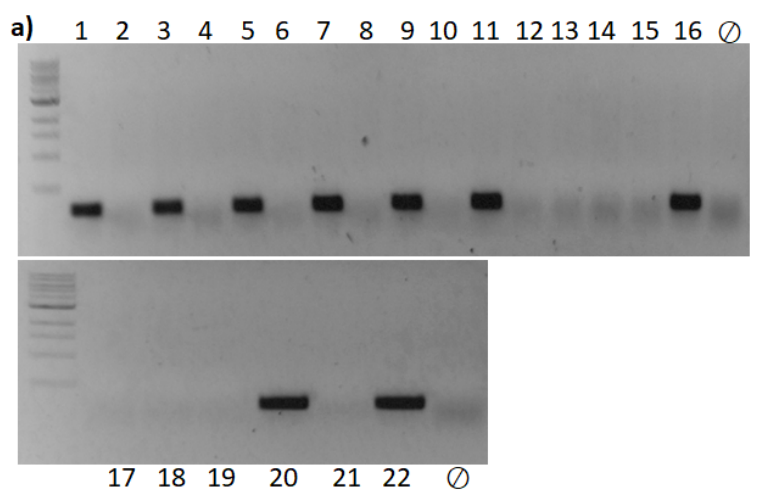

c)

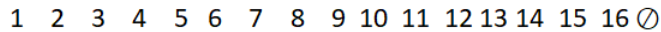
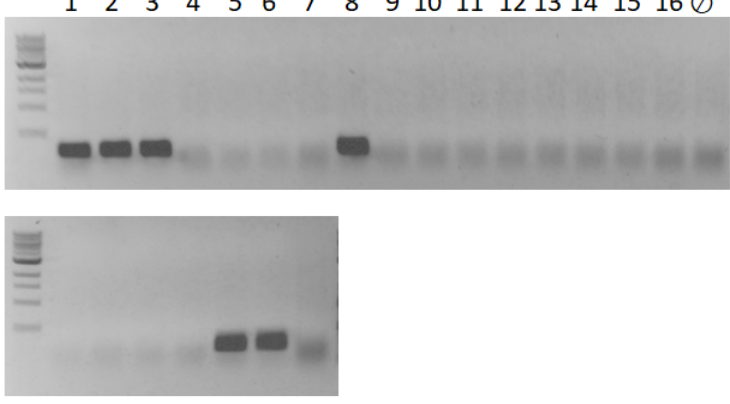

$171819202122 \varnothing$ b) ACGTGCCAACaCCaGTtGGaTGaGGGCTTGATG

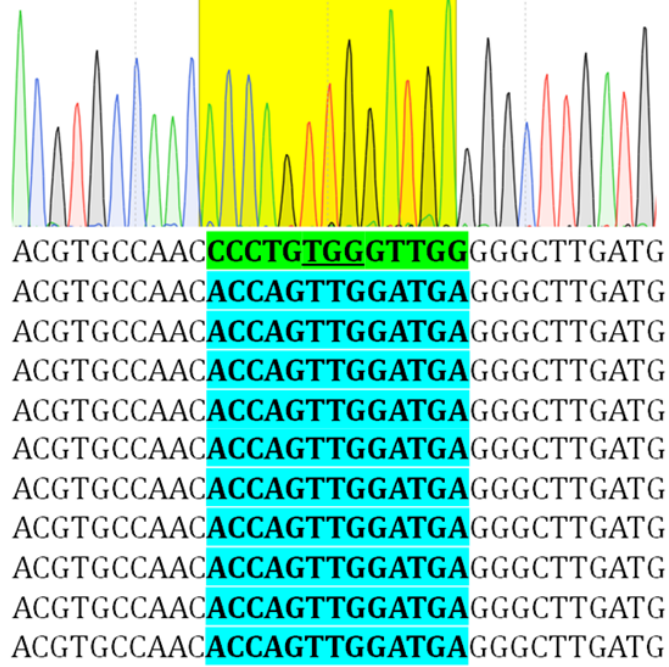

d)

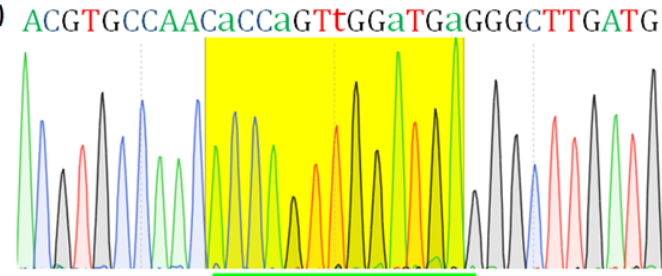

ACGTGCCAACCCCTGTGGGTTGGGGGCTTGATG ACGTGCCAACACCAGTTGGATGAGGGCTTGATG ACGTGCCAACACCAGTTGGATGAGGGCTTGATG ACGTGCCAACACCAGTTGGATGAGGGCTTGATG ACGTGCCAACACCAGTTGGATGAGGGCTTGATG ACGTGCCAACACCAGTTGGATGAGGGCTTGATG ACGTGCCAACACCAGTTGGATGAGGGCTTGATG ACGTGCCAACACCAGTTGGATGAGGGCTTGATG ACGTGCCAACACCAGTTGGATGAGGGCTTGATG ACGTGCCAACACCAGTTGGATGAGGGCTTGATG ACGTGCCAACACCAGTTGGATGAGGGCTTGATG

Figure 4. 14. Verification of exeM point mutation by recombineering combined with CRISPR/Cas9 counter-selection using different size oligos.

(a) 22 putative exeM mutants created using an oligo with 40nt of flanking homology arms were screened by colony PCR with a reverse primer specific to the DNA change introduced, validating the correct mutation in 9 colonies. (c) 22 putative exe $M$ mutants created using an oligo with 30nt of flanking homology arms were screened by colony PCR with a reverse primer specific to the DNA change introduced, validating the correct mutation in 6 colonies. (b, d) Sequence confirmation of 10 recombinants identified in (a and c, respectively), which revealed the point mutation TGG>TGA in amino acid 136, in addition to four consecutive wobble changes resulting in the DNA sequence 5'-ACCAGTTGGATGA-3' for all samples. $\oslash$ indicates a PCR negative control of the wild-type gene. The PAM sequence 5'-NGG-3' is underlined. 


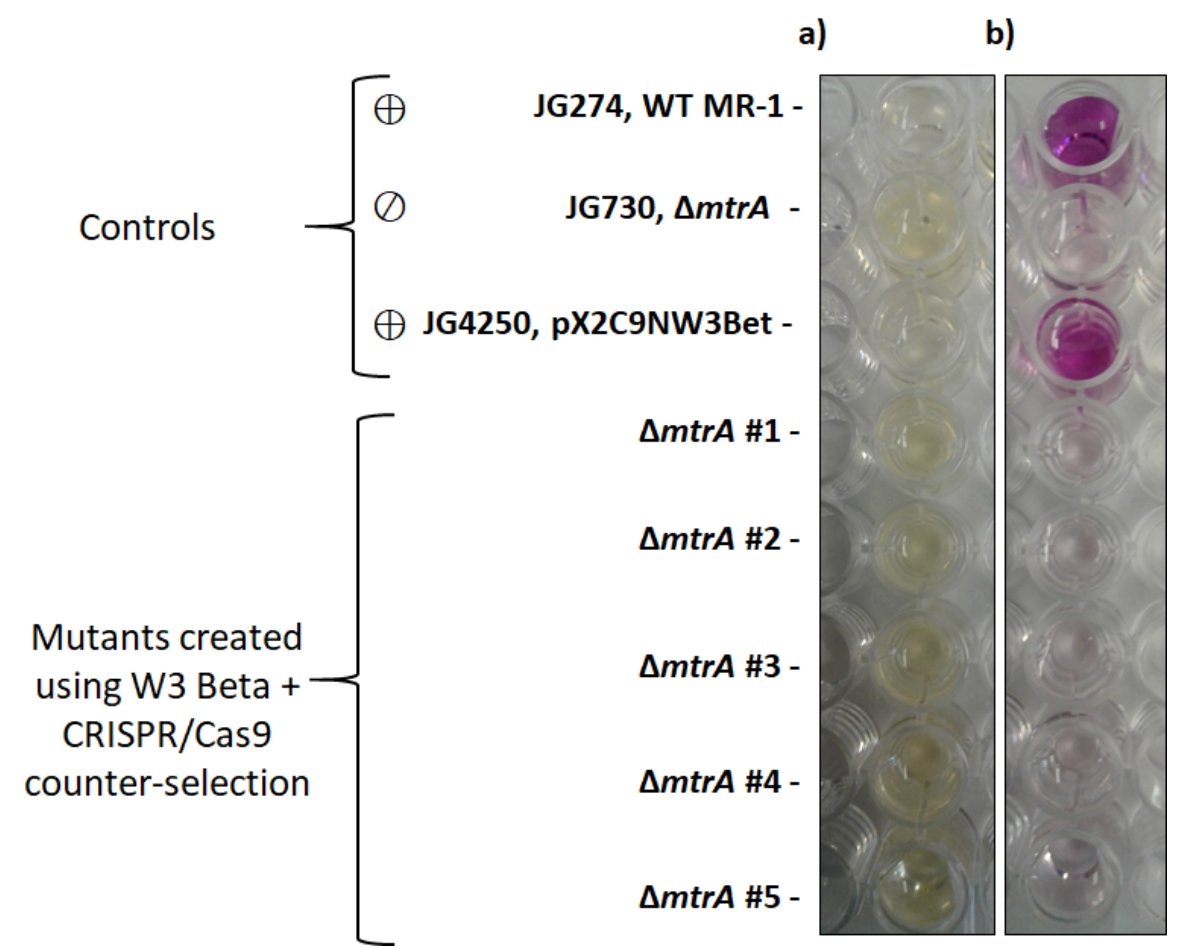

Figure 4. 15. Iron reduction test of $m \operatorname{tr} A$ deletion mutants.

(a) Iron citrate reduction and (b) ferrozine assay of $5 \mathrm{mtr} A$ mutants. $\oplus$ indicates a positive control able to reduce iron, $\oslash$ indicates a negative control unable to reduce iron.

\subsubsection{System optimization}

In order to optimize the expression of the W3 Beta recombinase and thus enhance homologous recombination in our system, we modified the DNA sequence located between the $\mathrm{P}_{\mathrm{Lac}}$ promoter and the RBS, upstream of the ORF encoding W3 Beta. Our original plasmid contained $49 \mathrm{bp}$ of a random sequence between the $\mathrm{P}_{\mathrm{Lac}}$ and the RBS (Figure 6A and Table S6). We deleted bases to make this sequence shorter, hoping to optimize transcription. For that purpose, we created two additional plasmid constructs, pX2C9pLac26 W3Bet and pX2C9pLac20w3Bet, which have 26bp and 20bp, respectively, between the $\mathrm{P}_{\mathrm{Lac}}$ and RBS (Figure 6A and Table S6).

Both of the new constructs resulted in a $>10$-fold increase in the frequency of recombination, while the same efficiency of $>90 \%$ was maintained. Specifically, $\mathrm{pX} 2 \mathrm{C} 9 \mathrm{pLac} 26 \mathrm{~W} 3 \mathrm{Bet}$ gave the best result with a frequency increase of $\simeq 30$-fold compared to the original editing plasmid. 
a)

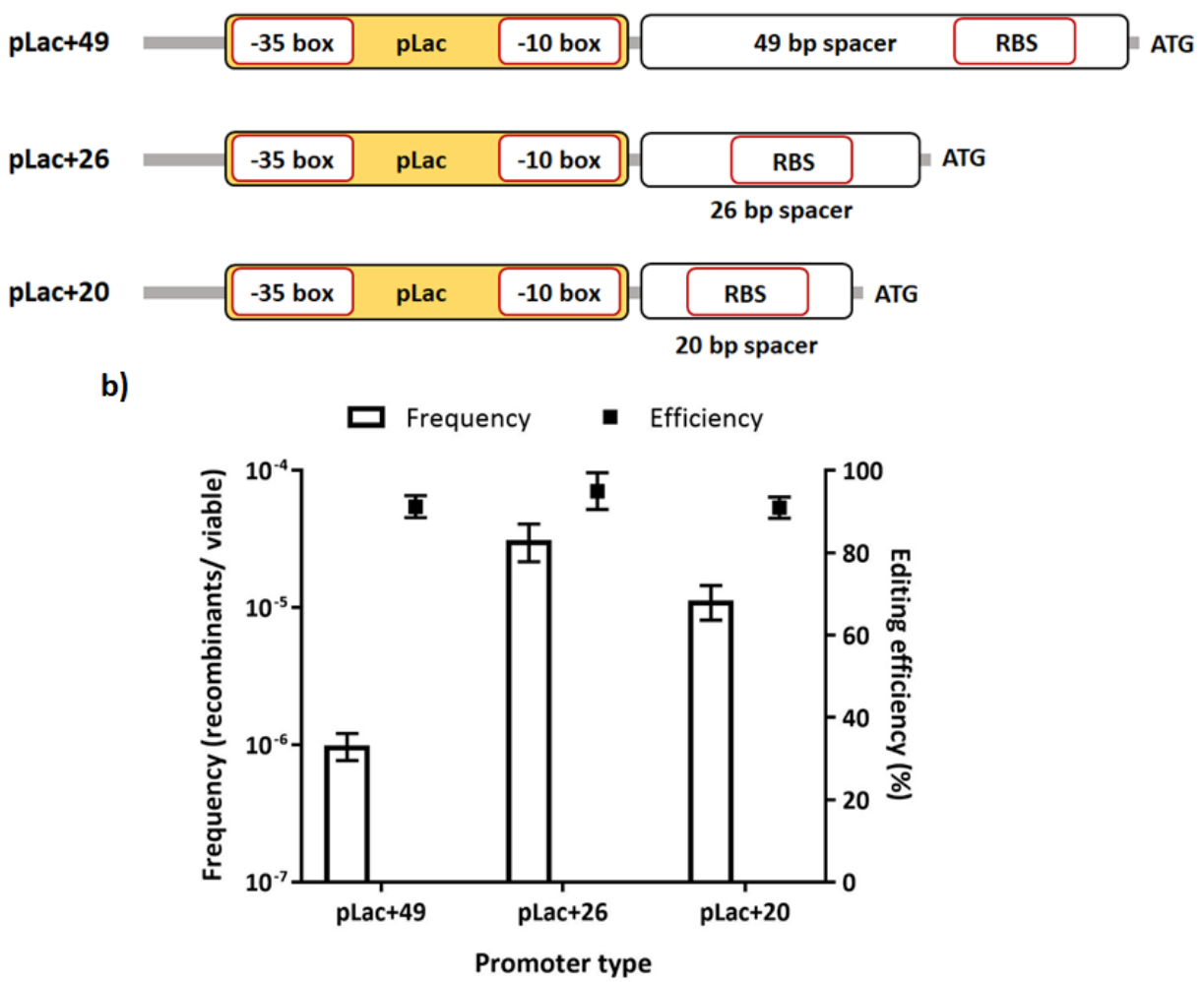

Figure 4. 16. System optimization.

a) Representation of the promoter and RBS upstream sequence of $s w 1153$ for each of the three plasmid constructs. b) Recombination frequencies of lac $Z$ in $S$. oneidensis using each plasmid construct. An oligo with 80nt of homology arms was used to target the lagging strand of lacZ. Cells harboring the corresponding editing plasmid were co-transformed with the sgRNA plasmid and ssDNA at a ratio $1 / 4$ and recovered for 2 hours after electroporation. Transformants were determined as total CFU growing on agar plates with both antibiotics, $\mathrm{Km}$ and $\mathrm{Cm}$, supplemented with arabinose and X-Gal. Viable cells were evaluated as CFUs growing on agar plates with $\mathrm{Km}$ and XGal only. Data shows averages of at least three independent experiments and error bars depict standard deviations.

Shewanella genome manipulation is limited by a lack of plasmid curing techniques and available inducible promoters for gene expression. Since most members of this family grow at low temperatures, high temperature-sensitive origins of replication cannot be used, thus development of better methods for conditional expression systems are needed. One option is the use of an additional sgRNA to kill the vector backbones, similar to the studies done by Jiang et. al. (Jiang et al. 2015) and Ronda et. al. (Ronda et al. 2016). The sgRNA could also be expressed from the same editing plasmid, although this strategy may be inconvenient for iterative genome-editing studies. 


\subsection{Discussion}

For more than 20 years, random mutagenesis has been the most powerful large scale technique to manipulate the Shewanella genome. Recently, the combination of recombineering with CRISPR/Cas9 as a counter-selection was shown to result in high efficiency of genome-editing in different bacteria (Jiang et al. 2015; Oh \& Van Pijkeren 2014; Sung et al. 2017; Aparicio et al. 2018; Penewit et al. 2018; Jiang et al. 2013), and has been adapted in E. coli for multiplex genome engineering such as the CRMAGE (CRISPR Optimized MAGE recombineering) study (Ronda et al. 2016), as well as for generation of high-throughput libraries using CREATE (CRISPR-enabled trackable genome engineering )(Garst et al. 2016). Although use of CRISPR/Cas9 for making mutations has been proven to be widely applicable to a variety of eukaryotes and prokaryotes (Hsu et al. 2015; Jiang \& Marraffini 2015; Komor et al. 2017; Mali et al. 2013; Cong et al. 2013), recombineering is more host-specific since it requires the use of bacteriophage proteins (Datta et al. 2008), however it allows creation of sophisticated, precise genetic editing. The phage recombinase proteins are generally single-stranded annealases, which target linear ssDNA to the lagging strand template of replication forks at gaps generated during discontinuous DNA replication (Ellis et al. 2001). In a recent study (Corts et al. 2019), we reported a new phagerecombinase, W3 Beta from Shewanella sp. W3-18-1, which gives a robust efficiency of precise mutagenesis (Corts et al. 2019), although a screen or selection is still needed to identify recombinants. Here, we have increased the ease of recombinant isolation by combining recombineering with the powerful technology CRISPR/Cas9 used as a counter-selection against non-recombinants. The result is highly efficient genome-editing that greatly expands the genetic engineering capabilities in Shewanella.

The minimal plasmid-based system that we have established may be broadly transferable to other related microbes, given that the origins of replication pBBR1 and p15a are functional. The editing plasmid contains Cas9 under the arabinose inducible $\mathrm{P}_{\mathrm{BAD}}$ promoter, with the downstream W3 Beta recombinase made constitutively from the lac promoter. The basic procedure has three steps. First, the editing plasmid is transferred into the bacterial cells to be modified. Next, a second plasmid encoding the targeting sgRNA and the linear ssDNA oligo containing the desired genetic changes are co-electroporated into these cells. Since the recombinase is constitutive, modification of the chromosomal target gene by W3 Beta using the ssDNA substrate occurs while the cells recover from electroporation. Finally, the cells are plated on solid agar medium containing arabinose, which induces the Cas9 nuclease. Cas9 introduces a double-strand break at unmodified DNA targets, leading to their degradation by cellular nucleases and allowing efficient elimination 
of the wild type genetic background. This system offers great advantages since the sgRNA can be programmed by simply inserting a 20nt short spacer into the sgRNA scaffold, and ssDNA oligos can be used for homologous recombination with flanking homology arms as short as 40nt. The only limitation is the presence of a nearby three nt PAM site (NGG) in the target sequence, which is necessary for Cas9 cleavage.

Three key elements of this system are: 1) Presence of the PAM site near the desired mutation site, since a silent PAM mutation is included in the recombinogenic ssDNA oligo to prevent Cas9 from cleaving the modified DNA sequence (Ronda et al. 2016; Garst et al. 2016). 2) To achieve high efficiencies with ssDNA recombineering, proper oligo design is critical. Care should be taken to locate the desired change centrally in the oligo. Use of a ssDNA oligo complementary to the lagging strand template of the target gene improves editing efficiency up to $\simeq 100$-fold compared to the leading strand (Ellis et al. 2001; Corts et al. 2019). Additionally, improved recombineering frequencies were obtained in E. coli when the host MMR was evaded by either making more than three consecutive mismatches or adding 4-5 wobble changes in codons adjacent to the desired mismatch (Sawitzke et al. 2011). 3) The cells should recover for several hours after electroporation and before CRISPR/Cas9 is induced with arabinose. This allows the recombination to occur and ensures that bacterial chromosomes have fully segregated before applying the negative selection (Ronda et al. 2016). In contrast to traditional targeted mutagenesis with suicide vectors or random mutagenesis techniques, our approach is simpler and less laborintensive. It will be useful for multiplex and/or iterative genome engineering, as shown in other hosts (Garst et al. 2016; Cong et al. 2013; Ronda et al. 2016), and should allow the introduction of many types of DNA changes at specific locations (Table 4.2).

In a recent study (Wu et al. 2019), Wu et al. report use of CRISPR/Cas9 and the RecET recombineering system from $E$. coli Rac prophage to precisely delete three genes in $S$. algae, using a plasmid-born $1 \mathrm{kbp}$ long DNA donor substrate introduced in the cell by conjugation. However, RecET recombination activity was not clearly demonstrated in this work, since controls omitting the phage recombination system were not presented. It is possible that the reported recombination was actually mediated by endogenous Shewanella recombination functions, since RecA proteins readily utilize homology arms of this length (Clark et al. 2014). We previously found that $E$. coli RecT is not functional in S. oneidensis (Corts et al. 2019), thus, W3 Beta is likely a better choice for homologous recombination in S. algae. Additionally, SpCas9 was expressed from the constitutive $S$. pyogenes promoter in this study, but controls to demonstrate Cas9 activity were lacking. We found that Cas9 must be tightly regulated since high Cas9 expression leads to a loss in cell viability, as shown in other bacteria (Cobb et al. 2014; Cui et al. 2018; Huang et al. 2015; Wang 
et al. 2018). CRISPRi was also recently applied in S. oneidensis for transient control of gene expression with the defective dCas9 protein (Cao et al. 2017), however, CRISPRi is not a tool for site-directed mutagenesis (Table 4.2).

Table 4. 2. Comparison of different genome editing techniques in Shewanella.

\begin{tabular}{|c|c|c|c|c|c|}
\hline Method & $\begin{array}{l}\text { Suicide- } \\
\text { plasmid } \\
\text { technique }\end{array}$ & $\begin{array}{l}\text { Transposon } \\
\text { mutagenesis }\end{array}$ & Recombineering & CRISPRi & $\begin{array}{l}\text { Recombineering } \\
\text { coupled to } \\
\text { CRISPR/Cas9 }\end{array}$ \\
\hline Purpose & Knock-out & $\begin{array}{l}\text { Random } \\
\text { mutagenesis }\end{array}$ & $\begin{array}{l}\text { Precise } \\
\text { mutations }\end{array}$ & $\begin{array}{l}\text { Regulation } \\
\text { of gene } \\
\text { expression }\end{array}$ & $\begin{array}{l}\text { Precise } \\
\text { mutations }\end{array}$ \\
\hline $\begin{array}{l}\text { Efficiency or } \\
\text { Frequency }\end{array}$ & $\simeq 10^{-4 \mathrm{a}}$ & $\simeq 10^{5 \mathrm{~b}}$ & $\simeq 5 \%{ }^{\mathrm{a}}$ & $0-180$-fold $^{\mathrm{c}}$ & $30->90 \%{ }^{\mathrm{d}}$ \\
\hline DNA delivery & conjugation & conjugation & electroporation & conjugation & electroporation \\
\hline Time spent & $\sim 2$ weeks & $\sim 1$ week & 4 days & $<1$ week & 4 days \\
\hline Labor & Intensive & Low & Low & Low & Low \\
\hline $\begin{array}{l}\text { Homology } \\
\text { arms for } \\
\text { recombination }\end{array}$ & $\simeq 1 \mathrm{kbp}$ & N/A & $40 \mathrm{nt}$ & N/A & $40 \mathrm{nt}$ \\
\hline $\begin{array}{l}\text { Plasmid } \\
\text { construction }\end{array}$ & $\begin{array}{l}\text { Multiple } \\
\text { PCR }\end{array}$ & No & No & $\begin{array}{l}\text { Golden } \\
\text { Gate } \\
\text { Assembly }\end{array}$ & $\begin{array}{l}\text { One step Gibson } \\
\text { Assembly }\end{array}$ \\
\hline $\begin{array}{l}\text { Possibility of } \\
\text { multiplexing }\end{array}$ & No & No & Yes & Yes & Yes \\
\hline $\begin{array}{l}\text { Genomic } \\
\text { sequence }\end{array}$ & Required & Not required & Required & Required & Required \\
\hline $\begin{array}{l}\text { Other } \\
\text { constrains }\end{array}$ & $\begin{array}{l}\text { Essential } \\
\text { genes } \\
\text { cannot be } \\
\text { mutated }\end{array}$ & $\begin{array}{l}\text { Essential } \\
\text { genes cannot } \\
\text { be mutated, } \\
\text { primarily } \\
\text { loss of } \\
\text { function } \\
\text { mutations }\end{array}$ & $\begin{array}{l}\text { Plasmid not yet } \\
\text { curable }\end{array}$ & $\begin{array}{l}\text { Polarity on } \\
\text { downstream } \\
\text { genes, } \\
\text { plasmid not } \\
\text { curable }\end{array}$ & $\begin{array}{l}\text { Plasmid not yet } \\
\text { curable }\end{array}$ \\
\hline
\end{tabular}

${ }^{\mathrm{a}}$ Measured as the number of recombinants among total viable cells (Saltikov \& Newman 2003; Blomfietd et al. 1991; Corts et al. 2019), ${ }^{\mathrm{b}} \mathrm{CFU} / \mu \mathrm{g}$ DNA in E. coli MG1655 (Gray et al. 2015; Yang et al. 2014), ${ }^{\mathrm{c}}$ Measured as repression of GFP expression (Peters et al. 2016; Cao et al. 2017), ${ }^{\mathrm{d}}$ Determined as the number of recombinants among the total surviving cells (this work). N/A: Not applicable

To our knowledge, the work presented here is the first demonstration of CRISPR/Cas9 used in conjunction with recombineering as a counter-selection in Shewanella. We have successfully made and isolated genetic recombinants that contain precise alteration of a single base pair as well as complete gene deletions in both $S$. oneidensis and $S$. amazonensis. We expect that this hybrid recombineering-CRISPR/Cas9 will be a transformative technology for Shewanella. Computational guide RNA design resources, as listed in Zhang's lab web (zlab.bio/guide-designresources), will ease the use of our technology. 
Similar to the high-throughput technologies developed in E. coli such as MAGE (Wang et al. 2009) and CREATE (Garst et al. 2016), our system will facilitate generation of comprehensive libraries large enough to interrogate the metabolism of Shewanella, facilitating better understanding of the EET capabilities and engineering of platform strains for use in biotechnology. We suspect that this method will become an essential tool for Shewanella researchers, and as the CRISPR/Cas technology advances, others will be able to quickly refine its applicability.

\subsection{Materials and Methods}

\subsubsection{Strains, plasmids, oligonucleotides and growth conditions}

Bacterial strains, plasmids and oligos used in this study are given in Table S1 and S2. E. coli UQ950 was used as a cloning host. E. coli methylation-deficient strains GM2163 and GM1674 were used to purify plasmids with $\mathrm{KmR}$ and $\mathrm{CmR}$, respectively, prior to electroporation in Shewanella. These strains contain a mutation in the DNA-cytosine methyltransferase $\left(\mathrm{dcm}^{--6}\right)$ that prevents methylation of cytosine residues, and thus, plasmids purified from these strains result in improved electro-transformation efficiency(Corts et al. 2019). S. oneidensis MR-1 and $S$. amazonensis SB2B were used for genome-editing purposes. E. coli was grown at $37^{\circ} \mathrm{C}$ and Shewanella at $30^{\circ} \mathrm{C}$ in LB. Oligonucleotides and gblocks were synthesized by Integrated DNA Technologies (IDT) and obtained as desalted. Km (50 $\mu \mathrm{g} / \mathrm{mL}$ for both species), Cm (30 $\mu \mathrm{g} / \mathrm{mL}$ for E. coli, $10 \mu \mathrm{g} / \mathrm{mL}$ for $S$. oneidensis and S. amazonensis $)$ X-gal $(60 \mu \mathrm{g} / \mathrm{mL}$ for S. oneidensis $)$ and Spect $(100 \mu \mathrm{g} / \mathrm{mL}$ for $S$. amazonensis) was added as needed. Antibiotic concentrations were reduced to $\mathrm{Km} 25 \mu \mathrm{g} / \mathrm{mL}$ and $\mathrm{Cm} 6.5 \mu \mathrm{g} / \mathrm{mL}$ when Shewanella strains harbored two plasmids. All strains were stored in $15 \%$ glycerol at $-80^{\circ} \mathrm{C}$. 
Table 4. 3. Strains and plasmids used in this study.

\begin{tabular}{|c|c|c|}
\hline Strain or plasmid & Genotype/relevant characteristics & Source \\
\hline \multicolumn{3}{|l|}{ S. oneidensis } \\
\hline JG274 & Wild type MR-1 & Gralnick lab \\
\hline JG3514 & $\mathrm{pX} 2$ Cas 9 & This study \\
\hline JG3638 & pdCas9 & This study \\
\hline JG3653 & pBTBX2 & (Corts et al. 2019) \\
\hline JG2150 & lacZ (integrated downstream of $g \operatorname{lm} S$ ) & (Corts et al. 2019) \\
\hline JG4250 & pX2C9pLacW3Bet & This study \\
\hline JG3675 & JG2150, pX2Cas9 & This study \\
\hline JG4226 & JG2150, pdCas 9 & This study \\
\hline JG4251 & JG2150, pX2C9pLacW3Bet & This study \\
\hline JG4284 & JG2150, pX2C9pLac26W3Bet & This study \\
\hline JG4285 & $\mathrm{JG} 2150, \mathrm{pX} 2 \mathrm{C} 9 \mathrm{pLac} 20 \mathrm{~W} 3 \mathrm{Bet}$ & This study \\
\hline \multicolumn{3}{|l|}{ S. amazonensis } \\
\hline JG98 & Wild type SB2B & Gralnick lab \\
\hline JG4232 & pACYC' & This study \\
\hline JG4233 & pdCas9 & This study \\
\hline JG4234 & $\mathrm{pX} 2 \mathrm{Cas} 9$ & This study \\
\hline JG4271 & pX2C9pLacW3Bet & This study \\
\hline \multicolumn{3}{|l|}{ E. coli } \\
\hline UQ950 & E. coli $\mathrm{DH} 5 \alpha \lambda$ (pir) host for cloning & $\begin{array}{l}\text { (Saltikov } \\
\text { Newman 2003) }\end{array}$ \\
\hline MG1655 & E. coli $\mathrm{F}-1$ lambda- $i l v G-r f b-50 r p h-1$ & Gralnick lab \\
\hline GM2163 & $\begin{array}{l}\text { dcm- dam- strain for electroporation, } \\
\text { CmR }\end{array}$ & CGSC\#: 6581 \\
\hline GM1674 & dcm- dam- strain for electroporation & CGSC\#7971 \\
\hline JG3939 & MG1655, pX2Cas9 & This study \\
\hline JG4236 & GM2163, pX2Cas9 & This study \\
\hline JG4122 & UQ950, pBTBX2 & (Corts et al. 2019) \\
\hline JG4059 & MG1655, pBTBX2 & (Corts et al. 2019) \\
\hline JG3649 & GM2163, pBTBX2 & (Corts et al. 2019) \\
\hline JG3938 & MG1655, pdCas9 & This study \\
\hline JG3656 & GM2163, pdCas 9 & This study \\
\hline JG4248 & UQ950, pX2C9pLacW3Bet & This study \\
\hline JG4249 & GM2163, pX2C9pLacW3Bet & This study \\
\hline JG4286 & UQ950, pX2C9pLac 26 W3Bet & This study \\
\hline JG4287 & UQ950, pX2C9pLac20 W3Bet & This study \\
\hline JG4228 & UQ950, pACYC & This study \\
\hline JG4229 & GM1674, pACYC' & This study \\
\hline JG4241 & UQ950, pACYC'-mtrAgRNA & This study \\
\hline JG4244 & GM1674, pACYC'-mtrAgRNA & This study \\
\hline JG4242 & UQ950, pACYC'-еxeMgRNA & This study \\
\hline JG4245 & GM1674, pACYC'-exeMgRNA & This study \\
\hline JG4048 & UQ950, pACYC'-lacZgRNA & This study \\
\hline JG4288 & GM1674, pACYC'-lacZgRNA & This study \\
\hline JG4243 & UQ950, pACYC'-sama_1213gRNA & This study \\
\hline JG4246 & GM1674, pACYC'-sama_1213gRNA & This study \\
\hline
\end{tabular}




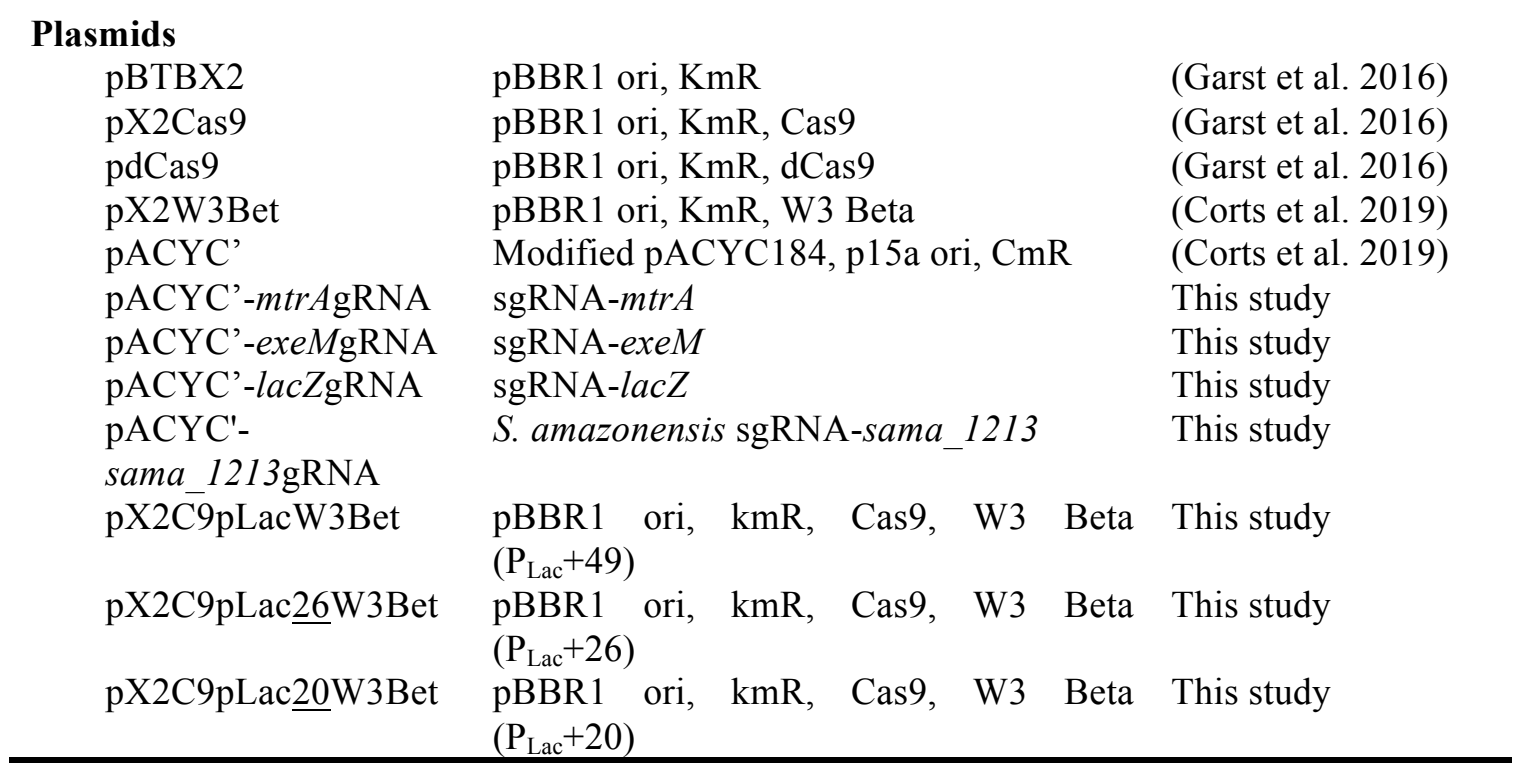


Table 4. 4. Primers used in this study.

\begin{tabular}{|c|c|c|}
\hline Primer & Sequence $\left(5^{\prime}-3^{\prime}\right)^{a}$ & Purpose \\
\hline \multicolumn{3}{|c|}{ Primers for plasmid modifications } \\
\hline ADC212 & $\begin{array}{l}\text { aaagcgetcacacagcctttagcttcttagTCTAGAGAA } \\
\text { TTCGTCAACGA }\end{array}$ & $\begin{array}{l}\text { Forward primer to linearize } \\
\text { pX2Cas9 for cloning in W3Beta } \\
\text { under } \mathrm{P}_{\mathrm{Lac}} \text {, with overhangs for } \\
\text { GA }\end{array}$ \\
\hline ADC213 & $\begin{array}{l}\text { aaaataggcgtatcacgaggccetttcgtcTCAGTCACC } \\
\text { TCCTAGCTGA }\end{array}$ & $\begin{array}{l}\text { Reverse primer to linearize } \\
\text { pX2Cas9 for cloning in W3Beta } \\
\text { under } \mathrm{P}_{\mathrm{Lac}} \text {, with overhangs for } \\
\text { GA }\end{array}$ \\
\hline ADC227 & $\begin{array}{l}\frac{\text { TTTATGCTTCCGGCTCGTATGTTGTGTG }}{\text { GATAATGGTTTCTTAGACGTCAGGTGG }} \\
\underline{\text { CACTAGGAGATATACCCATGGAAAAA }} \\
\text { CCAAAGCTAATCCAACG }\end{array}$ & $\begin{array}{l}\text { Forward primer to amplify } \\
\text { W3Beta with overhangs to } \\
\text { introduce } \mathrm{P}_{\mathrm{Lac}}\end{array}$ \\
\hline ADC228 & $\frac{\frac{\text { GACGAAAGGGCCTCGTGATACGCCTAT }}{\text { TTTTATAGGTTAATGTCATGATATTTAC }}}{\frac{\text { AC }}{\text { TTTATGCTTCCGGCTCGTATGTTGTG }}}$ & $\begin{array}{l}\text { Forward primer for a } 2^{\text {nd }} \text { round of } \\
\text { PCR of W3Beta with overhangs } \\
\text { to introduce a spacer between } \\
\text { Cas9 stop codon and } \mathrm{P}_{\mathrm{Lac}}\end{array}$ \\
\hline $\mathrm{ADC} 135$ & $\begin{array}{l}\text { CTA AGA AGC TAA AGG CTG TGT GAG } \\
\text { CG }\end{array}$ & $\begin{array}{l}\text { Reverse primer to amplify } \\
\text { W3Beta for cloning in pX2Cas } 9\end{array}$ \\
\hline ADC236 & $\begin{array}{l}\text { AGGAGATATACCCATGGAAAAACCAA } \\
\text { AGC }\end{array}$ & $\begin{array}{l}\text { Forward primer to modify } \\
\text { pX2C9pLacW3Beta to reduce the } \\
\text { space between } \mathrm{P}_{\text {Lac }} \text { and W3Beta } \\
\text { ORF by blunt ligation }\end{array}$ \\
\hline ADC235 & TCCACACAACATACGAGCCGG & $\begin{array}{l}\text { Reverse primer to modify } \\
\text { pX2C9pLacW3Beta to reduce the } \\
\text { space between } \mathrm{P}_{\text {Lac }} \text { and W3Beta } \\
\text { ORF to be } 20 \text { bp by blunt ligation }\end{array}$ \\
\hline $\mathrm{ADC} 237$ & CCATTATCCACACAACATACGAGCCG & $\begin{array}{l}\text { Reverse primer to modify } \\
\text { pX2C9pLacW3Beta to reduce the } \\
\text { space between } \mathrm{P}_{\text {Lac }} \text { and W3Beta } \\
\text { ORF to be } 26 \text { bp by blunt ligation }\end{array}$ \\
\hline
\end{tabular}

Primers for sequencing verification of pX2C9pLacW3Bet plasmid variants

ADC126 ATACCAGGATCTTGCCATCC

ADC122 GACGAAAGTAAACCCACTGG

ADC31 ATTAGCGGATCCTACCTGACG

To sequence verify araC

To sequence verify araC, $\mathrm{P}_{\mathrm{BAD}}$

$\mathrm{ADC} 127$

$\mathrm{ADC} 128$

GCGCATATGATTAAGTTTCGTGGTC

To sequence verify cas 9

To sequence verify cas 9

To sequence verify cas 9

ADC129

GTGAAGATTTGCTGCGCAAG

ADC130

AGATAGGGAGATGATTGAGG

ADC131

TCGTGGTAAATCGGATAACG

$\mathrm{ADC} 32$

TGTCAAGAAAACAGAAGTACAGAC

ADC202

TAAACGGATGCTGGCTAGTGC

ADC105

ATGGAAAAACCAAAGCTAATCCAACG

AAT CCC TGT GGT CAA GCT CG

To sequence verify cas 9

To sequence verify cas 9

To sequence verify cas 9

To sequence verify cas 9

To sequence verify $s w \_1153$

To sequence verify $s w_{-} 1153$

Primers for sgRNAs cloning and sequencing verification ADC7 ATGTTCCGGATCTGCATCGC

Forward primer to amplify sgRNA gblocks 


\begin{tabular}{|c|c|c|}
\hline ADC8 & CCG TGT GCT TCT CAA ATG CCT G & $\begin{array}{l}\text { Reverse primer to amplify } \\
\text { sgRNA gblocks }\end{array}$ \\
\hline ADC9 & GCG ATG CAG ATC CGG AAC AT & $\begin{array}{l}\text { Forward primer to amplify } \\
\text { pACYC' for sgRNA cloning }\end{array}$ \\
\hline $\mathrm{ADC} 10$ & CAG GCA TTT GAG AAG CAC ACG G & $\begin{array}{l}\text { Reverse primer to amplify } \\
\text { pACYC' for sgRNA cloning }\end{array}$ \\
\hline ADC11 & TGA GAG TCA ACG CCA TGA GC & $\begin{array}{l}\text { For sequencing sgRNAs after } \\
\text { cloning }\end{array}$ \\
\hline \multicolumn{3}{|c|}{ Primers for sequencing verifications of mutants } \\
\hline $\mathrm{ADC} 12$ & $\begin{array}{l}\text { CAG CTA GTA CAG ACT TCC ATT TCC } \\
\text { G }\end{array}$ & $\begin{array}{l}\text { Forward primer to sequence } \\
\text { verify mutations in } m t r A\end{array}$ \\
\hline $\mathrm{ADC} 13$ & CTG CAG TTA TGG CAT TAG TCG TC & $\begin{array}{l}\text { Reverse primer to sequence verify } \\
\text { mutations in } m t r A\end{array}$ \\
\hline $\mathrm{ADC} 14$ & $\begin{array}{l}\text { GGC AAT ACA GCA ATA GAT CTT ACC } \\
\text { GG }\end{array}$ & $\begin{array}{l}\text { Forward primer to sequence } \\
\text { verify mutations in exeM }\end{array}$ \\
\hline $\mathrm{ADC} 15$ & GGT TAC TTC GCT TTC AGA CTC G & $\begin{array}{l}\text { Reverse primer to sequence verify } \\
\text { mutations in exeM }\end{array}$ \\
\hline ADC51 & TGG CGT AAT AGC GAA GAG G & $\begin{array}{l}\text { Forward primer to sequence } \\
\text { verify mutations in lac } Z\end{array}$ \\
\hline ADC52 & CCT GAT CTT CCA GAT AAC TGC C & $\begin{array}{l}\text { Reverse primer to sequence verify } \\
\text { mutations in lacZ }\end{array}$ \\
\hline \multicolumn{3}{|c|}{ S. amazonensis mutants } \\
\hline ADC232 & CATGGCATTGCTGACACAGC & $\begin{array}{l}\text { Forward primer to sequence } \\
\text { verify mutations in sama_1213 }\end{array}$ \\
\hline ADC233 & GATCACCTTCGGTAAAGACGC & $\begin{array}{l}\text { Reverse primer to sequence verify } \\
\text { mutations in sama_1213 }\end{array}$ \\
\hline \multicolumn{3}{|c|}{ Primers for colony PCR screening of mutants } \\
\hline \multicolumn{3}{|c|}{ S. oneidensis mutants } \\
\hline ADC207 & GTGAGCGAGTAACAATTACTTATTA & $\begin{array}{l}\text { Reverse primer for colony PCR } \\
\text { screen of } 10 \mathrm{bp} \text { lac } Z \text { mutant }\end{array}$ \\
\hline ADC209 & CGAGCCGATGATCACTTAATAAGTAA & $\begin{array}{l}\text { Reverse primer for colony PCR } \\
\text { screen of } 10 \mathrm{bp} \text { mtrA mutant }\end{array}$ \\
\hline ADC48 & AGC CCC CAA CTT ACT CAT CA & $\begin{array}{l}\text { Reverse primer for colony PCR } \\
\text { screen of } 10 \mathrm{bp} \text { exe } M \text { mutant }\end{array}$ \\
\hline ADC238 & CAA GCC CTC ATC CAA CTG GT & $\begin{array}{l}\text { Reverse primer for colony PCR } \\
\text { screen of } 1 \text { bp exe } M \text { mutant }\end{array}$ \\
\hline ADC239 & TTCGGTGCAGTTGTTGATGG & $\begin{array}{l}\text { Forward primer for colony PCR } \\
\text { screen of } m t r A \mathrm{KO} \text { mutant }\end{array}$ \\
\hline ADC240 & GCTGTTATAACCGACACCGA & $\begin{array}{l}\text { Reverse primer for colony PCR } \\
\text { screen of } m t r A \text { KO mutant }\end{array}$ \\
\hline \multicolumn{3}{|c|}{ S. amazonensis mutants } \\
\hline ADC234 & GATCACCTTCGGTAAAGACGC & $\begin{array}{l}\text { Reverse primer for colony PCR } \\
\text { screen of 10bp sama_1213 } \\
\text { mutant }\end{array}$ \\
\hline
\end{tabular}

${ }^{a}$ Lower case designates Gibson assembly (GA) overhangs. Underline indicates a sequence overhang. 


\subsubsection{Plasmid construction}

All constructs in this study are given in Table S1. The ssDNA donor template sequences and the sgRNA, N20 sequences followed by the PAM are given in Tables S3 and S4, respectively, in the supplemental material. To check the specificity of sgRNA binding in the genome, a systematic BLAST search for the 10nt seed sequence adjacent to the PAM (full sequence: 5 '- $\mathrm{N}_{10^{-}}$ NGG-3', N being any nt) was performed to predict potential off-targets. Any sgRNA that mapped to multiple loci was discarded. Plasmids were extracted using a QIAprep kit (QIAGEN) according to the manufacturer's instructions. For PCR amplifications, Q5 DNA polymerase (NEB) was used.

The two-plasmid system, in which the Cas9 and W3 Beta are separated from the sgRNA, was used for genome editing as shown in Figure 4. Plasmid pX2C9pLacW3Bet consists of cas 9 under the $\mathrm{P}_{\mathrm{BAD}}$ promoter and $s w_{-} 1153$ under the $\mathrm{P}_{\text {Lac }}$ constitutive promoter, the broad-host range origin of replication pBBR1, araC and a $\mathrm{kmR}$ gene. pX2Cas9(Garst et al. 2016) was linearized by PCR, DpnI digested and ligated by Gibson assembly with the W3 Beta fragment. Sequencing confirmed the construct was mutation free.

Plasmid pACYC'(Corts et al. 2019), with a p15a origin of replication, was the vector backbone of choice for carrying the sgRNA, which is expressed under the lac promoter and was synthesized de novo. Four gblocks were ordered to target genes exeM (SO_1066), mtrA (SO_1777) and lacZ in S. oneidensis, and sama_1213 in S. amazonensis. The sequences of the gblocks are given in Table S5. Each gblock sequence had 20bp of homology to the pACYC' plasmid backbone for cloning by Gibson assembly. pACYC' was first linearized via PCR and DpnI digested. gblocks were resuspended following manufacturer's instructions and PCR amplified. PCR products were then PCR purified and cloned to form pACYC'-mtrAgRNA, pACYC'-exeMgRNA, pACYC'lacZgRNA and pACYC'-sama_1213gRNA.

Usually, ssDNA donor templates for recombineering were 170nt or 90nt long with the mutations located in the center of the sequence and 80nt or 40nt, respectively, of flanking homology to the target on each side of the mutation. For mtrA gene knock outs, the donor templates were $160 \mathrm{nt}, 120 \mathrm{nt}$ or $80 \mathrm{nt}$, containing $80 \mathrm{nt}$, $60 \mathrm{nt}$ or 40nt, respectively, of flanking homology outside of the ORF. For introducing $1 \mathrm{bp}$ change in exeM, the ssDNA template contained 4 wobble consecutive mutations in addition to the desired DNA change in the center, and either 40nt or 30nt flanking homology arms.

Blunt end ligation was used to shorten the space between the $\mathrm{P}_{\mathrm{Lac}}$ and the RBS of $\mathrm{s} w_{-} 1153$

in the pX2C9pLacW3Bet editing plasmid, forming pX2C9pLac20W3Bet and pX2C9pLac26W3Bet, with promoter sequences as shown in Table S6. pX2C9pLacW3Bet was 
linearized by PCR using primers ADC235-237 shown in Table S2, followed by DpnI digestion and blunt ligation using NEB T4 DNA ligase and T4 polynucleotide kinase. All plasmid clones were sequence verified to confirm the absence of mutations.

Table 4. 5. $P_{\text {Lac }}$ promoter sequences.

\begin{tabular}{|c|c|}
\hline Name & sequence $\left(5^{\prime}-3^{\prime}\right)^{\mathrm{a}, \mathrm{b}, \mathrm{c}}$ \\
\hline$\overline{\mathrm{pLac}+49}$ & $\begin{array}{l}\text { tttacactttatgcttccggetcgtatgttgtgtggataatggtttcttagacgtcaggtggcactAGGAGA } \\
\text { tatacccATG }\end{array}$ \\
\hline $\mathrm{pLac}+26$ & $\underline{\text { tttacactttatgcttccggctcgtatgttgtgtggataatggAGGAGAtatacccATG }}$ \\
\hline $\mathrm{pLac}+20$ & tttacactttatgcttccggctcgtatgttgtgtggaAGGAGAtatacccATG \\
\hline
\end{tabular}

\subsubsection{Electroporation}

Electroporation was performed at room temperature as previously reported(Corts et al. 2019). Cells were grown to the desired $\mathrm{OD}_{600}$, then three $\mathrm{ml}$ cells were spun by centrifugation at $7,607 \mathrm{rcf}$ for one minute. Three cell pellets were combined by resuspending them altogether with one $\mathrm{ml}$ of washing buffer, $1 \mathrm{M}$ sorbitol (pH 7.6). Cells were washed a total of three times prior to electroporation by centrifugation at 7,607 rcf for one minute. When using overnight cultures, only one $\mathrm{ml}$ cells was used and centrifugations were $3 \mathrm{~min}$. Cells were electroporated with DNA at 12 $\mathrm{kV} / \mathrm{cm}$ in $0.1 \mathrm{~cm}$ cuvettes. Following electroporation, $1 \mathrm{ml} \mathrm{L}$ broth was immediately added to the cuvette, and the mixture was transferred to a $2 \mathrm{~mL}$ tube for 2 hours outgrowth at $30^{\circ} \mathrm{C}$. All experiments were performed in triplicate. This protocol may need modification for use in other Shewanella strains.

\subsubsection{CRSPR/Cas9 activity assessment}

The efficiency of CRISPR/Cas9 cutting was determined by co-expressing a sgRNA and Cas9. All sgRNA expressing plasmids (250 ng) were electroporated into early exponential phase cells $\left(\mathrm{OD}_{600} \bumpeq 0.5\right)$ harboring either Cas9 (pX2Cas9) or dCas9 (pdCas9). Following electroporation, S. oneidensis cells were recovered for 4 hours, while $S$. amazonensis were recovered for 2 hours prior to plating on selective petri plates. In parallel, mock transformation controls lacking a sgRNA plasmid were performed to assess whether transformed cells would grow on selective plates with $\mathrm{Km}$ and $\mathrm{Cm}$. All sgRNA plasmids had been purified from methylation-deficient E. coli GM1674. 
Measurements to determine the viability of cells and transformation efficiency were performed in triplicate.

\subsection{5. ssDNA genome-editing combined with CRISPR/Cas9 counter-selection}

The sgRNA expressing plasmids were first co-electroporated with ssDNA donor templates at different ratios in $S$. oneidensis cells expressing Cas9 in the absence of a phage recombinase (Figure S4). Cells were grown to $\mathrm{OD}_{600} \bumpeq 0.5$ and electroporation was performed as explained above. Cells were recovered in one $\mathrm{ml} \mathrm{L}$ broth at $30^{\circ} \mathrm{C}$ for different times prior to plating on selection plates. Controls lacking sgRNA plasmid and ssDNA were performed to assess the number transformed cells that are spontaneously resistant to $\mathrm{Cm}$.

For genome editing using recombineering, all sgRNA expressing plasmids were coelectroporated with ssDNA donor templates at a ratio of $1 / 4$ (250 ng of gRNA plasmid and $1 \mu \mathrm{g}$ ssDNA) into $S$. oneidensis or S. amazonensis cells harboring both Cas9 and W3 Beta from $\mathrm{pX} 2 \mathrm{C} 9 \mathrm{pLacW} 3 \mathrm{Bet}$ (Figure 1). Cells were recovered in one $\mathrm{ml} \mathrm{L}$ broth at $30^{\circ} \mathrm{C}$ for 2 hours prior to plating. As controls, the same experiments were done without adding ssDNA donor template, to determine the number of colonies escaping from the Cas9-mediated DSB. At least three independent experiments were performed to determine recombination efficiencies. All sgRNA plasmids were purified from methylation-deficient E. coli GM1674. 
Table 4. 6. Oligonucleotides used for homologous recombination.

\begin{tabular}{|c|c|}
\hline Name & Sequence $\left(5^{\prime}-3^{\prime}\right)^{\mathrm{a}}$ \\
\hline \multicolumn{2}{|l|}{ S. oneidensis } \\
\hline 80nt-lag-lacZ & $\begin{array}{l}\text { 5' acggttacgatgegcccatctacaccaacgtgacctatcccattacggtcaatcegcegtttgttccc } \\
\text { acggagaatccgTAATAAGTAAttgttactcgctcacatttaatgttgatgaaagctggctaca } \\
\text { ggaaggccagacgcgaattatttttgatggegttaact }\end{array}$ \\
\hline 40nt- lag-lacZ & $\begin{array}{l}\text { 5'cattacggtcaatccgccgtttgttcccacggagaatccgTAATAAGTAAttgttactcgctc } \\
\text { acatttaatgttgatgaaagctggcta }\end{array}$ \\
\hline 80nt-lag-ехем & $\begin{array}{l}\text { 5'ttcccactgggcaggattaaagacagtatttgccactaatgagtcaagcttgcgcttaagggtaacat } \\
\text { caagccccaacTTACTCATCAaggggttggcacgtcaccaataatatcgacaaccgcacc } \\
\text { atctttaactaaggcaaccgcatcgccaccgttaaaactta }\end{array}$ \\
\hline 40nt-lag-exeM-1bp & $\begin{array}{l}\text { 5'ctaatgagtcaagcttgcgcttaagggtaacatcaagcccTcaTccAacTggTgttggcacgtc } \\
\text { accaataatatcgacaaccgcaccatctt }\end{array}$ \\
\hline 30nt-lag-exeM-1bp & $\begin{array}{l}\text { 5'aagcttgcgcttaagggtaacatcaagcccTcaTccAacTggTgttggcacgtcaccaataata } \\
\text { tcgacaacc }\end{array}$ \\
\hline 80nt-lag-mtr $A$ & $\begin{array}{l}\text { 5'caatggetggectgcaatgtgaggcatgccacggcccactgggtcagcacaacaaaggcggcaa } \\
\text { cgagccgatgatcactTAATAAGTAAtaagcaatcaaccttaagtgccgacaagcaaaacag } \\
\text { cgtatgtatgagctgtcaccaagacgataagcgtatgtcttgga }\end{array}$ \\
\hline 80nt-lag-mtrA-KO & $\begin{array}{l}\text { 5'gtgaaaatgtaatttgcccaagcagggggagctcgetcccctttcttgaattttgttgggacaaattg } \\
\text { ggaagcctattggagacgagaaaatgaaatttaaactcaatttgatcactctagcgttattagccaacac } \\
\text { aggcttggecgtcgetgetga }\end{array}$ \\
\hline 60nt-lag-mtr $A-\mathrm{KO}$ & $\begin{array}{l}\text { 5'agcagggggagctcgctcccctttcttgaattttgttgggacaaattgggaagcctattggagacga } \\
\text { gaaaatgaaatttaaactcaatttgatcactctagegttattagccaacaca }\end{array}$ \\
\hline 40nt-lag-mtr $A$-KO & $\begin{array}{l}\text { 5'cctttcttgaatttgttgggacaaattgggaagcctattggagacgagaaatgaaatttaaactcaat } \\
\text { ttgatcactc }\end{array}$ \\
\hline \multicolumn{2}{|l|}{ S. amazonensis } \\
\hline $\begin{array}{l}\text { 80nt-lag- } \\
\text { SAMA_1213 }\end{array}$ & $\begin{array}{l}\text { 5'cccaggcttccgtggggattgtggcagtcactgcacaccatctggccgttttcgactggatgactgg } \\
\text { agcgettgtggatTTACTTATTAccttttgttcgagatgacacgcagtacacacctcgacttcgg } \\
\text { tggetctttgctgaaccggatccttggcagtatggatc }\end{array}$ \\
\hline $\begin{array}{l}\text { 40nt-lag- } \\
\text { SAMA_1213 }\end{array}$ & $\begin{array}{l}\text { 5'tctggecgttttcgactggatgactggagcgettgtggatTTACTTATTAcettttgttcgaga } \\
\text { tgacacgcagtacacacctcgacttc }\end{array}$ \\
\hline
\end{tabular}

${ }^{\mathrm{a}}$ Uppercase designates the mutations introduced. 
Table 4. 7. Spacers used for sgRNA/Cas9 targeting.

\begin{tabular}{lc}
\hline Name & 20nt spacer sequence +PAM (5'-3') \\
\hline S. oneidensis & \\
SO_mtrA-gRNA & AACGAGCCGATGATCACTTTTGG \\
SO_exeM-gRNA & TGGTGACGTGCCAACCCCTGTGG \\
SO_lacZ-gRNA & GTTCCCACGGAGAATCCGACEGG \\
S. amazonensis & \\
sama_1213-gRNA & ACTGGAGCGCTTGTGGATATCGG \\
\hline
\end{tabular}

Table 4. 8. gBLOCKS containing the synthetic sgRNA sequences.

\begin{tabular}{|c|c|}
\hline Name & sequence $\left(5^{\prime}-3^{\prime}\right)^{\mathrm{a}}$ \\
\hline \multicolumn{2}{|l|}{ S. oneidensis } \\
\hline SO_mtrA-gRNA & $\begin{array}{l}\text { ATGTTCCGGATCTGCATCGCtttacactttatgcttccggctcgtatgttgtgtggaac } \\
\text { gagccgatgatcactttgttttagagctagaaatagcaagttaaaataaggetagtcegttatcaacttg } \\
\text { aaaaagtggcaccgagtcggtgcttttttCAGGCATTTGAGAAGCACACGG }\end{array}$ \\
\hline SO_exeM-gRNA & $\begin{array}{l}\text { ATGTTCCGGATCTGCATCGCtttacactttatgcttccggctcgtatgttgtgtggtggt } \\
\text { gacgtgccaacccetggttttagagetagaaatagcaagttaaaataaggetagtccgttatcaacttg } \\
\text { aaaaagtggcaccgagtcggtgcttttttCAGGCATTTGAGAAGCACACGG }\end{array}$ \\
\hline SO_lacZ-gRNA & $\begin{array}{l}\text { ATGTTCCGGATCTGCATCGCtttacactttatgcttccggctcgtatgttgtgtgggttc } \\
\text { ccacggagaatccgacgttttagagctagaaatagcaagttaaaataaggctagtcegttatcaacttg } \\
\text { aaaaagtggcaccgagtcggtgcttttttCAGGCATTTGAGAAGCACACGG }\end{array}$ \\
\hline \multicolumn{2}{|l|}{ S. amazonensis } \\
\hline sama_1213-gRNA & $\begin{array}{l}\text { ATGTTCCGGATCTGCATCGCtttacactttatgcttccggctcgtatgttgtgtggact } \\
\text { ggagcgcttgtggatatgttttagagctagaaatagcaagttaaaataaggctagtccgttatcaacttg } \\
\text { aaaaagtggcaccgagtcggtgcttttttCAGGCATTTGAGAAGCACACGG }\end{array}$ \\
\hline
\end{tabular}

\subsubsection{Screening for edited cells}

Successful recombination of ssDNA donor template with the bacterial chromosome was first screened directly on $\mathrm{Km}$ and $\mathrm{Cm}$ agar petri plates supplemented with $0.8 \mathrm{mM}(0.01 \%)$ arabinose. In all experiments, uninduced controls were plated on the same medium but lacking arabinose to evaluate plasmid transformation efficiency. For lacZ editing, the agar plates also contained $60 \mu \mathrm{g} / \mathrm{mL} \mathrm{X-Gal} \mathrm{and} \mathrm{editing} \mathrm{efficiency} \mathrm{was} \mathrm{assessed} \mathrm{by} \mathrm{the} \mathrm{proportion} \mathrm{of} \mathrm{white} \mathrm{colonies}$ over the total. When targeting exeM, mtrA and sama_1213, the editing efficiency was assessed with a colony PCR screen of $>20$ colonies from at least two independent experiments. Primers specific to the mutation introduced were used for colony PCR (Figure 5). Mutations were additionally verified by Sanger sequencing. 


\section{CHAPTER V: CONCLUSIONS AND FUTURE DIRECTIONS}

In this dissertation, I introduced and demonstrated utility of new experimental strategies that should accelerate efforts to investigate and engineer Shewanella. In Chapter II, I described development of a highly efficient electro-transformation protocol for S. oneidensis MR-1 and showed its utility in other Shewanella strains. In Chapter III, I described the characterization of a new prophage recombinase for recombineering in Shewanella and demonstrate its ability to recombine ssDNA oligos, targeting the bacterial genome. In Chapter IV, I demonstrated the use of CRISPR/Cas9 as a counter-selection and showed that recombineering coupled to CRISPR/Cas9 results in extremely high efficiency of genome editing of Shewanella. Each of these strategies has limitations, as well as promising future directions that could accelerate our ability to manipulate Shewanella. Below, I discuss the drawbacks and next steps for each of these strategies

\subsection{Application of electrotransformation in different Shewanella strains}

The ability to perform electro-transformation for transferring DNA into cells should benefit many genome engineering endeavors. Most high-throughput applications developed in E. coli combine parallel DNA synthesis with electroporation as means to transfer massive DNA libraries and to use recombineering for rapid genome manipulations. Circular plasmid DNA or pools of linear DNA cassettes or oligos can be transferred en masse using electroporation, similar to the studies performed using TRMR (Trackable Multiplex Recombineering) (Warner et al. 2010), MAGE (Multiplex Automated Genome Engineering) (Wang et al. 2009) and CREATE (CRISPR EnAbled Trackable Genome Engineering) (Garst et al. 2016). Warner et al., used TRMR to demonstrate the construction of two comprehensive E. coli genomic libraries comprising 8,000 distinct mutations and gene-trait mapping of these cells under different environments. Wang et al., used MAGE to optimize the expression of 24 genes in parallel for the improvement of lycopene production. Garst et al., used CREATE to generate over 50,000 mutants in E. coli and to map the sequence-activity relationship of the variants for a range of applications. These studies highlight the importance and simplicity with which genome engineers can massively transfer DNA into a cell population using electroporation. Therefore, the ability to electro-transform DNA into 
Shewanella should accelerate efforts to engineer this microbe for a wide range of industrial applications.

A limitation of this methodology, however, is the transferability to other organisms and even strains within the same species. Different strains have diverse characteristics, and our ability to manipulate them would be valuable from both a microbiology and biotechnology perspective. Could we apply electroporation for high efficiency of DNA transfer in other Shewanella? One major limitation is the host restriction-modification system, which can be evaded by using nonmethylated DNA purified from methylation-deficient E. coli. However, as demonstrated in this dissertation, many other factors play a role as well, such as optical density, washing buffer and more, for obtaining the high efficiency required for large-scale studies. Improved strategies for parallel electroporation of various samples should facilitate development of electroporation in multiple strains and organisms.

\subsection{Use of recombineering for different types of DNA changes}

With recombineering a much higher range of mutations can be achieved compared to traditional deletion libraries using transposons. Recombineering allows scientists to precisely introduce any DNA change into the chromosome of a bacterial cell; insertions, point mutations and deletions, using linear DNAs(Sharan et al. 2009; Baba et al. 2006; Ellis et al. 2001; Sawitzke et al. 2011). Wang et al. used MAGE to introduce a pool of ssDNA mutagenic oligos for recombineering in $E$. coli using the $\lambda$-Red system. Because of the simplicity of using ssDNA, they were able to introduce successful genetic modifications in $>30 \%$ of the population every 2.5 hours. Thus, recombineering is a powerful technology for rapidly generating a library of mutants. Usually, these recombinases are host specific (Datta et al. 2008), however, as described in this dissertation I found that the Shewanella recombinase W3 Beta seems to be broader host than $\lambda$-Beta or RecT, since it was functional in $E$. coli at the same high efficiency as $\lambda$-Beta, while $\lambda$-Beta and RecT were not functional in $S$. oneidensis. It would be of interest to other microbiologists and genetic engineers to know whether W3 Beta is also active in other hosts.

The current bottleneck is the ability to introduce larger insertions, such as an entire gene or cluster of genes. For that purpose, dsDNA recombineering is preferred as longer synthetic DNA sequences are less economic and chemical synthesis is size limited, but recombineering requires a functional phage exonuclease for recombination of dsDNA (Sharan et al. 2009), as well as the recombinase protein. Adjacent to the ORF encoding W3 Beta is a putative exonuclease, perhaps functionally associated with W3 Beta. Characterization of this exonuclease in conjunction with W3 
Beta may open the possibility to transfer larger DNA cassettes by dsDNA recombineering and, consequently, allow integration of exogenous pathways for genetic analysis or strain improvement in Shewanella.

\subsection{CRISPR/Cas9 counter-selection}

The use CRISPR/Cas9 of as a counter-selection tool coupled with recombineering provides a great advantage for bacterial genome engineering, specially, when the organism is unable to otherwise repair the double-stranded breaks created by Cas9, since this results in a very high efficiency of selection against unmodified chromosomes (Selle \& Barrangou 2015). A big limitation, however, is the ability to cure the plasmid backbones at the end of the experiment in order to obtain a plasmid-free cell. Since most Shewanella grow at lower temperatures (Hau \& Gralnick 2007), it would be beneficial to engineer replication origins sensitive at a lower temperature range similar to the $\mathrm{pS} 101$ origin, which is sensitive at the high temperature of $37^{\circ} \mathrm{C}$.

In this work, I show high efficiency functionality of CRISPR/Cas9 in both S. oneidensis and S. amazonensis, thus expanding the genetic tools of different Shewanella. However, other strains have fewer tools available. An option for engineering other strains is to combine the expression of the sgRNA and Cas9 in one plasmid in order to easily test the efficiency of the system, although this strategy would not allow for iterative studies. Since S. amazonensis also grows at $37^{\circ} \mathrm{C}$, future experiments could be performed in this strain using a gRNA expressing plasmid with the pS101 origin for iterative editing, for example.

\subsection{Large-scale genome engineering using recombineering coupled to CRISPR/Cas9}

Recombineering coupled to CRISPR/Cas9 counter-selection allows an extremely high efficiency of genome-editing in Shewanella. This technology, as it currently is demonstrated in this dissertation, could be used to develop a small library to engineer the active sites of essential proteins from the metal respiratory pathway and, by growing the variants in different environments, we could gain further knowledge on the mechanism of metal respiration, for example. However, usually high-throughput or multiplex studies are desired to investigate several genotypes en masse and/or in parallel, similar to the CREATE (Garst et al. 2016) and MAGE (Wang et al. 2009) studies, respectively, which require not only high efficiency but also high frequency of editing. The efficiency depends on how well the CRISPR/Cas9 counter-selection performs, so that the final population contains as few unmodified cells as possible. However, the frequency depends on: 1) 
the effectiveness of DNA transfer and, 2) the probability of recombinant formation using recombineering.

In this dissertation, I maximized electro-transformation of $S$. oneidensis, obtaining $\sim 10^{6}$ transformants/ ug DNA and $\sim 10^{8}$ transformants/ ug DNA when cells are harvested at early $\left(\mathrm{OD}_{600} \sim 0.4-0.5\right)$ and late $\left(\mathrm{OD}_{600} \sim 2\right)$ exponential phase. Because recombineering exploits replication forks (Thomason et al. 2016), transformation of early exponential phase cells are required, which I showed results in a maximum efficiency of $\sim 5 \% S$. oneidensis recombinants among the total viable cells transformed. Yet, for recombineering coupled to CRISPR/Cas9 as described here by co-electroporation of ssDNA and gRNA plasmid, recombinants on the order of $10^{2}-10^{3}$ per $\mathrm{mL}$, depending on the gene targeted, are obtained. In order to increase the probability of recombination, I enhanced transcription of the recombinase by shortening the sequence space between the $\mathrm{P}_{\mathrm{Lac}}$ promoter and RBS upstream of the recombinase encoding gene. This modification resulted in a $\sim 30$-fold increase in frequency. Nevertheless, this frequency is still $\sim 10^{3}$ orders of magnitude lower than the maximum recombineering efficiency. This difference may be due to the inefficiency in co-transformation of multiple different DNA molecules versus a single molecule electroporation, but possibly other factors such as the difference in promoter type and plasmid copy number and/or stability. Although our system was developed without strain engineering, rational removal of single- and double- strand exonucleases could potentially enhance the frequency of recombineering as shown in $V$. cholera (Dalia et al. 2017) and E. coli (Mosberg et al. 2012; Costantino \& Court 2003; Li et al. 2013).

In the CREATE study (Garst et al. 2016), three different plasmids were used for expressing Cas9, $\lambda$-Red and the CREATE cassette. However, having several plasmids increases the metabolic burden of the cell and it makes it more challenging to implement in other hosts. In this dissertation, I designed this genome-editing technology so that only two vectors are required; one expresses the gRNA and the other contains Cas9 made inducible from the $\mathrm{P}_{\mathrm{BAD}}$ promoter and the W3 Beta encoding gene under $\mathrm{P}_{\mathrm{LAC}}$, which is constitutive in Shewanella. The latter plasmid is designed such that both Cas9 and W3 Beta ORFs share the same terminator. Ideally, however, W3 Beta should be made also inducible for tight control over each part of the system. This along with further improvements, especially with the ability to perform dsDNA recombineering and curing of the vector backbones for iterative experiments, will help build larger-scale technologies similar to the CREATE and MAGE studies in E. coli (Garst et al. 2016; Wang et al. 2009).

Multiplex iterative engineering could allow the rapid design of a strain with various reduction abilities of different compounds simultaneously, for instance, by engineering of specific promoters and/or RBSs for up-regulation of essential pathways required for metal and oxygen 
respiration. On the other hand, implementation of a CREATE library for saturation mutagenesis of the entire Mtr pathway, for example, could provide a way to deeply interrogate the mechanism of metal reduction and improve the metal reduction capabilities of Shewanella. In all, the technology shown in this thesis should have an impact on genome-engineering of Shewanella and, potentially, other less well-studied microbes. Improvements that could enable this technology to be used for large-scale is just a matter of time. 


\section{REFERENCES}

Altenbuchner, J., 2016. Editing of the Bacillus subtilis Genome by the CRISPR-Cas9 System. Applied and environmental microbiology, 82(17), pp.5421-5427.

Aparicio, T., de Lorenzo, V. \& Martínez-García, E., 2018. CRISPR/Cas9-Based Counterselection Boosts Recombineering Efficiency in Pseudomonas putida. Biotechnology Journal, 13(5), pp.1-10.

Baba, T. et al., 2006. Construction of Escherichia coli K-12 in-frame, single-gene knockout mutants: the Keio collection. Molecular systems biology, 2, p.2006.0008.

Beblawy, S. et al., 2018. Extracellular reduction of solid electron acceptors by Shewanella oneidensis. Molecular Microbiology, 109, pp.571-583.

Beliaev, A.S. \& Saffarini, D. A, 1998. Shewanella putrefaciens mtrB Encodes an Outer Membrane Protein Required for Fe(III) and Mn(IV) Reduction. J. Bacteriol, 180(23), pp.6292-6297.

Bendall, M.L. et al., 2013. Exploring the roles of DNA methylation in the metal-reducing bacterium Shewanella oneidensis MR-1. Journal of Bacteriology, 195(21), pp.4966-4974.

Biswas, A. et al., 2016. CRISPRDetect: A flexible algorithm to define CRISPR arrays. BMC Genomics, 17(356), pp.1-14.

Blomfietd, I.C. et al., 1991. Allelic exchange in Escherichia coli using the Bacillius subtilis sacB gene and a temperature-sensitive pSC101 replicon. Molecular Microbiology, 5(6), pp.14471457.

Boyle, N.R. et al., 2013. Recombineering to homogeneity: Extension of multiplex recombineering to large-scale genome editing. Biotechnology Journal, 8(5), pp.515-522.

Breuer, M. et al., 2015. Multi-haem cytochromes in Shewanella oneidensis MR-1: structures, functions and opportunities. JR Soc Interface, 12.

Brutinel, E.D. \& Gralnick, J. A., 2012a. Anomalies of the anaerobic tricarboxylic acid cycle in Shewanella oneidensis revealed by Tn-seq. Molecular Microbiology, 86(2), pp.273-283.

Brutinel, E.D. \& Gralnick, J. a., 2012b. Shuttling happens: Soluble flavin mediators of extracellular electron transfer in Shewanella. Applied Microbiology and Biotechnology, 93(1), pp.41-48.

Bryan, A. \& Swanson, M.S., 2011. Oligonucleotides stimulate genomic alterations of Legionella pneumophila. Molecular Microbiology, 80(1), pp.231-247.

Cao, Y. et al., 2017. CRISPRi - sRNA: Transcriptional - Translational Regulation of Extracellular Electron Transfer in Shewanella oneidensis. ACS Synthetic Biology, 6, pp.1679-1690.

Choi, K.H., Kumar, A. \& Schweizer, H.P., 2006. A 10-min method for preparation of highly electrocompetent Pseudomonas aeruginosa cells: Application for DNA fragment transfer 
between chromosomes and plasmid transformation. Journal of Microbiological Methods, 64(3), pp.391-397.

Chu, V.T. et al., 2015. Increasing the efficiency of homology-directed repair for CRISPR-Cas9induced precise gene editing in mammalian cells induced precise gene editing in mammalian cells. Nature biotechnology, 33, pp.543-548.

Clark, I.C. et al., 2014. Chlorate reduction in Shewanella algae ACDC is a recently acquired metabolism characterized by gene loss, suboptimal regulation and oxidative stress. Molecular Microbiology, 94(1), pp.107-125.

Cobb, R.E., Wang, Y. \& Zhao, H., 2014. High-Efficiency Multiplex Genome Editing of Streptomyces Species Using an Engineered CRISPR/Cas System. ACS Synthetic Biology, p.141208152636004.

Cong, L. et al., 2013. Multiplex Genome Engineering Using CRISPR / Cas Systems. Science, 339(6121), pp.819-823.

Corts, A.D. et al., 2019. A new recombineering system for precise genome-editing in Shewanella oneidensis strain MR-1 using single-stranded oligonucleotides. Scientific Reports, 9(39), pp. $1-10$.

Costantino, N. \& Court, D.L., 2003. Enhanced levels of lambda Red-mediated recombinants in mismatch repair mutants. Proceedings of the National Academy of Sciences of the United States of America, 100(26), pp.15748-15753.

Coursolle, D. \& Gralnick, J.A., 2010. Modularity of the Mtr respiratory pathway of Shewanella oneidensis strain MR-1. Molecular Microbiology, 77(July), pp.995-1008.

Cui, L. et al., 2018. A CRISPRi screen in E. coli reveals sequence-specific toxicity of dCas9. Nature Communications, 9(1912), pp.1-10.

Cui, L. \& Bikard, D., 2016. Consequences of Cas9 cleavage in the chromosome of Escherichia coli. Nucleic acids research, 44(9), pp.4243-4251.

Dalia, T.N. et al., 2017. Enhancing multiplex genome editing by natural transformation (MuGENT) via inactivation of ssDNA exonucleases. Nucleic Acids Research, 45(12), pp.7527-7537.

Datsenko, K. a \& Wanner, B.L., 2000. One-step inactivation of chromosomal genes in Escherichia coli K-12 using PCR products. Proceedings of the National Academy of Sciences of the United States of America, 97(12), pp.6640-6645.

Datta, S. et al., 2008. Identification and analysis of recombineering functions from Gram-negative and Gram-positive bacteria and their phages. Proceedings of the National Academy of Sciences of the United States of America, 105(5), pp.1626-1631.

Deutschbauer, A. et al., 2011. Evidence-based annotation of gene function in Shewanella 
oneidensis MR-1 using genome-wide fitness profiling across 121 conditions. PLoS Genetics, $7(11)$.

Ellis, H.M. et al., 2001. High efficiency mutagenesis, repair, and engineering of chromosomal DNA using single-stranded oligonucleotides. Proceedings of the National Academy of Sciences, 98(12), pp.6742-6746.

Fredrickson, J.K. et al., 2008. Towards environmental systems biology of Shewanella. Nature reviews. Microbiology, 6, pp.592-603.

$\mathrm{Fu}$, J. et al., 2012. Full-length RecE enhances linear-linear homologous recombination and facilitates direct cloning for bioprospecting. Nature Biotechnology, 30(5), pp.440-448.

Garst, A.D. et al., 2016. Genome-wide mapping of mutations at single-nucleotide resolution for protein, metabolic and genome engineering. Nature Biotechnology, (December), pp.1-14.

Gray, A.N. et al., 2015. High-throughput bacterial functional genomics in the sequencing era. Current Opinion in Microbiology, 27, pp.86-95.

Groh, J.L. et al., 2007. Genes That Enhance the Ecological Fitness of Shewanella oneidensis MR1 in Sediments Reveal the Value of Antibiotic Resistance. Applied and Environmental Microbiology, 73(2), pp.492-498.

Hau, H.H. \& Gralnick, J. A, 2007. Ecology and biotechnology of the genus Shewanella. Annual review of microbiology, 61(August), pp.237-58.

Heidelberg, J.F. et al., 2002. Genome sequence of the dissimilatory metal ion-reducing bacterium Shewanella oneidensis. Nature biotechnology, 20, pp.1118-1123.

Hossain, M.J. et al., 2015. Genome modifications and cloning using a conjugally transferable recombineering system. Biotechnology Reports, 8, pp.24-35.

Hsu, P.D., Lander, E.S. \& Zhang, F., 2015. Development and Applications of CRISPR-Cas9 for Genome Engineering. Cell, 157(6), pp.1262-1278.

$\mathrm{Hu}$, S. et al., 2014. Genome engineering of Agrobacterium tumefaciens using the lambda Red recombination system. Applied Microbiology and Biotechnology, 98(5), pp.2165-2172.

Huang, H. et al., 2015. One-step high-efficiency CRISPR/Cas9-mediated genome editing in Streptomyces. Acta Biochimica et Biophysica Sinica, 47(4), pp.231-243.

Huang, Y. et al., 2017. Recombineering using RecET in Corynebacterium glutamicum ATCC14067 via a self-excisable cassette. Scientific Reports, 7(1), p.7916.

Jiang, W. et al., 2013. RNA-guided editing of bacterial genomes using CRISPR-Cas systems. Nature biotechnology, 31(3), pp.233-9. A

Jiang, W. \& Marraffini, L.A., 2015. CRISPR-Cas : New Tools for Genetic Manipulations from Bacterial Immunity Systems. Annual review of microbiology, 69, pp.209-28. 
Jiang, Y. et al., 2015. Multigene editing in the Escherichia coli genome via the CRISPR-Cas9 system. Applied and Environmental Microbiology, 81(7), pp.2506-2514.

Jinek, M. et al., 2012. A Programmable Dual-RNA - Guided Endonuclease in Adaptive Bacterial Immunity. Science, 337(6096), pp.816-822.

Jones, M.E. et al., 2010. Shewanella oneidensis MR-1 mutants selected for their inability to produce soluble organic-Fe ( III ) complexes are unable to respire Fe ( III ) as anaerobic electron acceptor. Environmental Microbiology, 12(4), pp.938-950.

Karvelis, T., Gasiunas, G. \& Siksnys, V., 2017. Harnessing the natural diversity and in vitro evolution of Cas9 to expand the genome editing toolbox. Current Opinion in Microbiology, 37, pp.88-94.

van Kessel, J.C. \& Hatfull, G.F., 2007. Recombineering in Mycobacterium tuberculosis. Nature methods, 4(2), pp.147-52.

Komor, A.C., Badran, A.H. \& Liu, D.R., 2017. CRISPR-Based Technologies for the Manipulation of Eukaryotic Genomes. Cell, 168, pp.20-36.

Kuei, T.-K. \& Puchta, H., 2019. CRISPR / Cas-mediated gene targeting in plants : finally a turn for the better for homologous recombination. Plant Cell Reports, 38(4), pp.443-453.

Lee, H.H. et al., 2017. Recombineering in Vibrio natriegens. bioRxiv, p.130088.

Lee, J.K. et al., 2018. Directed evolution of CRISPR-Cas9 to increase its specificity. Nature Communications, 9(3048), pp.1-10.

Li, X.T. et al., 2013. Bacterial DNA polymerases participate in oligonucleotide recombination. Molecular Microbiology, 88(5), pp.906-920.

Liang, G. et al., 2016. Selection of highly efficient sgRNAs for CRISPR / Cas9-based plant genome editing. Scientific Reports, 6(21451), pp.1-8.

Liang, L. et al., 2017. CRISPR EnAbled Trackable genome Engineering for isopropanol production in Escherichia coli. Metabolic Engineering, 41, pp.1-10.

Liu, R. et al., 2018. Iterative genome editing of Escherichia coli for 3-hydroxypropionic acid production. Metabolic Engineering, 47, pp.303-313.

Lovley, D.R., 2012. Electromicrobiology. Annual review of microbiology, (66), pp.391-409.

Macaluso, A. \& Mettus, A., 1991. Efficient transformation of Bacillus thuringiensis requires nonmethylated plasmid DNA. Journal of Bacteriology, 173(3), pp.1353-1356.

Makarova, K.S. et al., 2012. Evolution and classification of the CRISPR-Cas systems. Nature reviews. Microbiology, 9(6), pp.467-477.

Mali, P. et al., 2013. RNA-Guided Human Genome Engineering via Cas9. Science, (February), pp.823-827. 
Modrich, P., 1991. Mechanisms and Biological Effects of Mistmatch Repair. Annual review of genetics, (25), pp.229-253.

Mosberg, J.A. et al., 2012. Improving Lambda Red Genome Engineering in Escherichia coli via Rational Removal of Endogenous Nucleases. PLoS ONE, 7(9), pp.1-12.

Mougiakos, I. et al., 2017. Efficient Genome Editing of a Facultative Thermophile Using Mesophilic spCas9. ACS Synthetic Biology, (6), pp.849-861.

Mougiakos, I. et al., 2016. Next Generation Prokaryotic Engineering : The CRISPR-Cas Toolkit. Cell, 34(7), pp.575-587.

Murphy, K.C., 1998. Use of bacteriophage $\lambda$ recombination functions to promote gene replacement in Escherichia coli. Journal of Bacteriology, 180(8), pp.2063-2071.

Murphy, K.C., 1991. $\lambda$ Gam protein inhibits the helicase and $\chi$-stimulated recombination activities of Escherichia coli RecBCD enzyme. Journal of Bacteriology, 173(18), pp.5808-5821.

Muyrers, J.P.P. et al., 2000. RecE/ RecT and Red $\alpha / \operatorname{Red} \beta$ initiate double stranded break repair by specifically interacting with their respective partners. Genes Dev, 14, pp.1971-1982.

Myers, C.R. \& Myers, J.M., 1997. Replication of plasmids with the p15A origin in Shewanella putrefaciens MR-1. Letters in applied microbiology, 24(3), pp.221-5.

Myers, C.R. \& Nealson, K.H., 1988. Bacterial manganese reduction and growth with manganese oxide as the sole electron acceptor. Science (New York, N.Y.), 240(4857), pp.1319-1321.

Ng, H. \& Dean, N., 2017. Dramatic Improvement of CRISPR/Cas9 Editing in Candida albicans by Increased Single Guide RNA Expression. Molecular Biology and Physiology, 2(2), pp.117.

Oh, J.H. \& Van Pijkeren, J.P., 2014. CRISPR-Cas9-assisted recombineering in Lactobacillus reuteri. Nucleic Acids Research, 42(17), pp.1-11.

Oppenheim, A.B. et al., 2004. In vivo recombineering of bacteriophage $\lambda$ by PCR fragments and single-strand oligonucleotides. , 319(Virology), pp.185-189.

Paix, A., Schmidt, H. \& Seydoux, G., 2016. Cas9-assisted recombineering in C . elegans: genome editing using in vivo assembly of linear DNAs. Nucleic acids research, 44(15), pp.1-9.

Penewit, K. et al., 2018. Efficient and scalable precision genome editing in Staphylococcus aureus through conditional recombineering and CRISPR/Cas9-mediated counterselection. mBio, 9(1), pp.1-14.

Peters, J.M. et al., 2016. A Comprehensive, CRISPR-based Functional Analysis of Essential Genes in Bacteria. Cell, 165(6), pp.1493-1506.

Peters, J.M. et al., 2015. Bacterial CRISPR: Accomplishments and Prospects. Current Opinion in Microbiology, 27, pp.121-126. 
Pines, G. et al., 2015. Bacterial recombineering - genome engineering via phage-based homologous recombination. ACS Synthetic Biology, p.150409125239003.

Prior, J.E., Lynch, M.D. \& Gill, R.T., 2010. Broad-host-range vectors for protein expression across gram negative hosts. Biotechnology and Bioengineering, 106(2), pp.326-332.

Qiu, X. et al., 2004. Survival of Shewanella oneidensis MR-1 after UV Radiation Exposure. , 70(11), pp.6435-6443.

Rabaey, K. \& Verstraete, W., 2005. Microbial fuel cells: Novel biotechnology for energy generation. Trends in Biotechnology, 23(6), pp.291-298.

Rachkevych, N. et al., 2014. Improving the efficiency of plasmid transformation in Shewanella oneidensis MR-1 by removing ClaI restriction site. Journal of Microbiological Methods, 99(1), pp.35-37.

Rath, D. et al., 2015. The CRISPR-Cas immune system : Biology , mechanisms and applications. Biochimie, 117, pp.119-128.

Reisch, C.R. \& Prather, K.L.J., 2015. The no-SCAR ( Scarless Cas9 Assisted Recombineering ) system for genome editing in Escherichia coli. Nature Scientific Reports, 5(15096), pp.1-12.

Richardson, C.D. et al., 2016. Enhancing homology-directed genome editing by catalytically active and inactive CRISPR-Cas9 using asymmetric donor DNA. Nature biotechnology, 34(3), pp.339-345.

Ronda, C. et al., 2016. CRMAGE: CRISPR Optimized MAGE Recombineering. Scientific Reports, 6 (December 2015), pp.1-11.

Ryan, D.E. et al., 2018. Improving CRISPR - Cas specificity with chemical modifications in singleguide RNAs. Nucleic acids research, 46(2), pp.792-803.

Saffarini, D. A et al., 1994. Anaerobic Respiration Of Shewanella putrefaciens Requires Both Chromosomal And Plasmid-Borne Genes. FEMS Microbiology Letters, 119(3), pp.271-277.

Saltikov, C.W. \& Newman, D.K., 2003. Genetic identification of a respiratory arsenate reductase. Proceedings of the National Academy of Sciences, 100(19), pp.10983-10988.

Satomi, M., 2014. The Family Shewanellaceae. In The Prokaryotes. Fourth Edi. E. Rosenberg et al., eds., Springer Heidelberg New York Dordrecht London.

Sawitzke, J.A. et al., 2011. Probing cellular processes with oligo-mediated recombination and using the knowledge gained to optimize recombineering. Journal of Molecular Biology, 407(1), pp.45-59.

Selle, K. \& Barrangou, R., 2015. Harnessing CRISPR-Cas systems for bacterial genome editing. Trends in Microbiology, 23(4), pp.225-232.

Shah, S.A. et al., 2013. Protospacer recognition motifs: Mixed identities and functional diversity. 
RNA Biology, 10(5), pp.891-899.

Sharan, S.K. et al., 2009. Recombineering: a homologous recombination-based method of genetic engineering. Nature protocols, 4(2), pp.206-223.

Shi, L. et al., 2016. Extracellular electron transfer mechanisms between microorganisms and minerals. Nature reviews. Microbiology, 14(10), pp.651-662.

Shi, L. et al., 2012. Molecular underpinnings of Fe(III) oxide reduction by Shewanella oneidensis MR-1. Frontiers in Microbiology, 3(FEB), pp.1-10.

Shi, L. et al., 2005. Overexpression of multi-heme C-type cytochromes. BioTechniques, 38(2), pp.297-299.

Shuman, H.A. \& Silhavy, T.J., 2003. Microbial genetics: The art and design of genetic screens: Escherichia coli. Nature Reviews Genetics, 4(6), pp.419-431.

Stout, E.A. et al., 2018. Deletion-based escape of CRISPR-Cas9 targeting in Lactobacillus gasseri. Microbiology, 164, pp.1098-1111.

Sung, J. et al., 2017. CRISPR / Cas9-coupled recombineering for metabolic engineering of Corynebacterium glutamicum. Metabolic Engineering, 42(June), pp.157-167.

Swingle, B., Markel, E., et al., 2010. Oligonucleotide recombination in Gram-negative bacteria. Molecular Microbiology, 75(1), pp.138-148.

Swingle, B., Bao, Z., et al., 2010. Recombineering using recTE from Pseudomonas syringae. Applied and Environmental Microbiology, 76(15), pp.4960-4968.

Thomason, L.C., Costantino, N. \& Court, D.L., 2016. Examining a DNA Replication Requirement for Bacteriophage $\lambda$ Red- and Rac Prophage RecET-Promoted Recombination in Escherichia coli. mBio, 7(5), pp.e01443-16.

Tiedje, J.M., 2002. Shewanella - the environmentally versatile genome. Nature biotechnology, 20, pp.1093-94.

Tong, Y. et al., 2015. CRISPR-Cas9 Based Engineering of Actinomycetal Genomes. ACS Synthetic Biology, 4(9), pp.1020-1029.

$\mathrm{Tu}$, Q. et al., 2016. Room temperature electrocompetent bacterial cells improve DNA transformation and recombineering efficiency. Scientific Reports, 6 (December 2015), pp.18.

$\mathrm{Tu}, \mathrm{Z}$. et al., 2005. An improved system for competent cell preparation and high efficiency plasmid transformation using different Escherichia coli strains. , 8(1).

Venkateswaran, K. et al., 1999. Polyphasic taxonomy of the genus Shewanella and description of Shewanella oneidensis sp . now. , 49(2), pp.705-724.

Wang, B. et al., 2018. A RecET - assisted CRISPR - Cas9 genome editing in Corynebacterium 
glutamicum. Microbial Cell Factories, 17(63), pp.1-16.

Wang, H.H. et al., 2009. Programming cells by multiplex genome engineering and accelerated evolution. Nature, 460(7257), pp.894-898.

Warner, J.R. et al., 2010. Rapid profiling of a microbial genome using mixtures of barcoded oligonucleotides. Nature biotechnology, 28(8), pp.856-862.

Weinstock, M.T. et al., 2016. Vibrio natriegens as a fast-growing host for molecular biology. Nature Methods, 13(10), pp.849-851.

Wendt, K.E. et al., 2016. CRISPR/Cas9 mediated targeted mutagenesis of the fast growing cyanobacterium Synechococcus elongatus UTEX 2973. Microbial cell factories, 15(115), pp.1-8.

Weninger, A. et al., 2016. Combinatorial optimization of CRISPR/Cas9 expression enables precision genome engineering in the methylotrophic yeast Pichia pastoris. Journal of Biotechnology, 235, pp.139-149.

Wolf, T. et al., 2016. Targeted genome editing in the rare actinomycete Actinoplanes sp. SE50/110 by using the CRIPSR/Cas9 System. Journal of Biotechnology, 231, pp.122-128.

Wu, N. et al., 2010. Enhancing DNA electrotransformation efficiency in Escherichia coli DH10B electrocompetent cells. Electronic Journal of Biotechnology, 13(5), pp.1-9.

$\mathrm{Wu}, \mathrm{Z}$. et al., 2019. Reversal of carbapenem-resistance in Shewanella algae by CRISPR /Cas9 genome editing. Journal of Advanced Research, 18, pp.61-69.

Yan, M. et al., 2017. CRISPR-Cas12a-Assisted Recombineering in Bacteria. Applied and Environmental Microbiology, 83(17), pp.1-13.

Yang, H. et al., 2014. Genome-Scale Metabolic Network Validation of Shewanella oneidensis Using Transposon Insertion Frequency Analysis. PLoS Computational Biology, 10(9), pp.110 .

Yang, P., Wang, J. \& Qi, Q., 2015. Prophage recombinases-mediated genome engineering in Lactobacillus plantarum. Microbial Cell Factories, 14(1), pp.1-11.

Yang, Y. et al., 2013. Roles of UndA and MtrC of Shewanella putrefaciens W3-18-1 in iron reduction. BMC Microbiology, 13(1).

Yin, J. et al., 2015. A new recombineering system for Photorhabdus and Xenorhabdus. Nucleic Acids Research, 43(6), p.e36.

Yu, D. et al., 2000. An efficient recombination system for chromosome engineering in Escherichia coli. Proceedings of the National Academy of Sciences of the United States of America, 97(11), pp.5978-5983.

Zhang, Y. et al., 1998. A new logic for DNA engineering using recombination in Escherichia coli. 
Nature genetics, 20(2), pp.123-128.

Zhou, Y. \& Ng, I.S., 2016. Explored a cryptic plasmid pSXM33 from Shewanella xiamenensis $\mathrm{BC} 01$ and construction as the shuttle vector. Biotechnology and Bioprocess Engineering, 21(1), pp.68-78. 